Precision electroweak physics at high energies

This article has been downloaded from IOPscience. Please scroll down to see the full text article.

2010 Rep. Prog. Phys. 73126201

(http://iopscience.iop.org/0034-4885/73/12/126201)

View the table of contents for this issue, or go to the journal homepage for more

Download details:

IP Address: 141.34.3.101

The article was downloaded on 15/02/2011 at $14: 34$

Please note that terms and conditions apply. 


\title{
Precision electroweak physics at high energies
}

\author{
Sabine Riemann \\ DESY, Platanenallee 6, D-15378 Zeuthen, Germany \\ E-mail: sabine.riemann@desy.de \\ Received 16 November 2009, in final form 4 August 2010 \\ Published 19 November 2010 \\ Online at stacks.iop.org/RoPP/73/126201
}

\begin{abstract}
The era of precision electroweak measurements at high energies started twenty years ago with the electron-positron colliders LEP and SLC. Excellent performance of accelerators, advanced detectors and precise theoretical predictions led to enormous progress in the examination of the Standard Model. Ongoing measurements at the Tevatron complement the precision results from LEP and SLC and allow a comprehensive test of the electroweak interactions. So far, the Standard Model has agreed well with the measurements and no significant deviation has been observed. But the Higgs boson, an important constituent of the Standard Model, has not yet been found but its mass is constrained by the high precision data. In this report an overview of the global electroweak tests of the Standard Model is presented. It includes the measurements, and their treatment to probe the Standard Model with highest precision. Finally, a short outlook to physics scenarios beyond the Standard Model is given.
\end{abstract}

(Some figures in this article are in colour only in the electronic version)

This article was invited by P Zerwas.

\section{Contents}

1. Introduction

2. The Standard Model

2.1. The building blocks of the Standard Model

2.2. Radiative corrections

2.3. Input parameters for Standard Model analyses

3. Precision observables

3.1. Fermion-pair cross section

3.2. Photon radiation

3.3. Z boson widths

3.4. Asymmetries in fermion-pair production

3.5. Pseudo-observables and model-dependence of the analyses

4. Measurements at $\mathbf{e}^{+} \mathbf{e}^{-}$colliders

4.1. Precise luminosity measurement at LEP

4.2. Energy measurement at LEP

4.3. Polarization measurement at SLC

4.4. Measurements at future $e^{+} e^{-}$colliders

5. Precision measurements at the $Z$ resonance

5.1. Measurement of the $Z$ lineshape

5.2. Measurement of forward-backward charge asymmetries

5.3. The parameters of the $Z$ lineshape
5.4. Tau polarization at LEP 14

5.5. Polarized asymmetries at SLC 15

5.6. Heavy quark flavours at LEP and SLC 16

5.7. Inclusive hadronic charge asymmetry 17

6. Parameters of the $\mathbf{Z}$ boson 18

6.1. The $Z$ boson mass 18

6.2. The Z boson partial widths 18

6.3. The number of light neutrinos 18

6.4. Effective couplings of the neutral weak current 19

6.5. The effective weak mixing angle 20

6.6. Sensitivity to radiative corrections beyond $Q E D$

7. Global test of the Standard Model

7.1. Hadronic vacuum polarization 21

7.2. Fit to Z pole data 22

7.3. W boson production at LEP-II 23

7.4. W boson measurements at the Tevatron 24

7.5. World average of W boson mass measurement 25

7.6. Top quark production at the Tevatron 25

7.7. Constraints from measurements at low energies 26

7.8. Global electroweak fit 27

7.9. Global fit and the mass of the Higgs boson 29 
7.10. Future precision measurements at the $Z$ pole

7.11. Parametrization of radiative corrections

8. $\mathbf{e}^{+} \mathbf{e}^{-}$annihilation at energies above the $Z$ boson

8.1. Fermion-pair production above the $Z$ pole

8.2. Interpretation of LEP-II fermion-pair production measurements

9. Beyond the Standard Model
9.1. Supersymmetry 34

9.2. Models with extra dimensions 34

9.3. Strong electroweak symmetry breaking 35

9.4. New gauge bosons 36

10. Summary and outlook 37

Acknowledgments $\quad 37$

$\begin{array}{ll}\text { References } & 37\end{array}$

\section{Introduction}

The Standard Model of particles and forces represents our present knowledge of particle physics. It generalizes and extends the quantum theory of electromagnetism to include the weak interactions, and it provides a theory to describe successfully the strong force. The Standard Model is certainly one of the greatest achievements in physics in the 20th century. At the beginning of the 1970s its theoretical foundations were established. The framework as introduced by Sheldon Glashow, Abdus Salam and Steven Weinberg, unifies electromagnetism and weak forces [1]. The model possesses charged and neutral field quanta, indicating that in addition to the known weak charged currents also weak neutral currents, i.e. weak processes without switching electric charges, should exist. When the theory took shape, weak neutral currents had never been observed. The very short range of the weak force implies massive particles mediating the weak force in contrast to the massless photon, the carrier of the electromagnetic force with infinite range. The difference in masses is attributed to spontaneous symmetry breaking which is implemented into the model using the Higgs mechanism. With their pioneering theoretical work Gerard 't Hooft and Martinus Veltman showed in the early 1970s how to use the electroweak model to make precise calculations of particle properties and interactions [2]. The discovery of the predicted weak neutral currents in neutrino scattering by the Gargamelle collaboration in 1973 $[3,4]$ was the first important step to confirm the electroweak part of the Standard Model experimentally. The second step followed a decade later when the massive weak gauge bosons $\mathrm{W}^{ \pm}$and $\mathrm{Z}[5,6]$ were discovered in proton-antiproton collisions in 1983 at CERN. Furthermore, the Standard Model spectrum of fermions classified into three generations had been almost completed and verified experimentally except for the top quark. The experimental proof of the theory at the quantum level was the next logical step, and two $\mathrm{e}^{+} \mathrm{e}^{-}$ colliders, the Large Electron Positron (LEP) collider at CERN and the Stanford Linear Collider (SLC) at SLAC, were built to produce copious numbers of $\mathrm{Z}$ bosons allowing precise measurements of its properties. Before LEP and SLC started operating in 1989, the masses of the $\mathrm{W}$ and $\mathrm{Z}$ bosons had been measured to an accuracy of a few hundred megaelectron volts; the electroweak mixing parameter, $\sin ^{2} \theta_{\mathrm{W}}$, related to the mass ratio of $\mathrm{W}$ and $\mathrm{Z}$ boson and describing the part of weak interaction in neutral current processes, had been determined at the per cent level. The existence of the top quark could be inferred by measuring the quantum numbers of its isospin partner, the b-quark.
In the 1990s, about 18 million $\mathrm{Z}$ bosons were collected by the experiments at LEP and SLC. Based on this impressive amount of data precision measurements of the $\mathrm{Z}$ boson properties were performed and confronted with predictions of the Standard Model. In order to meet this experimental precision, the uncertainty of theoretical predictions has been continually reduced. Thus, the electroweak sector of the Standard Model has been successfully confirmed at the permille level and the key parameters of the Standard Model have been measured with high precision. This yielded important conclusions, in particular two famous results: the first concerns the number of families in the Standard Model. By comparing the total $\mathrm{Z}$ boson width as measured in the Breit-Wigner lineshape with the visible lepton and quark decay channels, the number of light neutrino species has been determined to be three, corresponding to three families of matter particles. The second result is the determination of the top quark mass. Virtual top quarks affect the masses and the couplings of the electroweak gauge bosons, and so the mass had been predetermined with amazing accuracy before the discovery of the top quark at the Tevatron, a proton-antiproton collider. The perfect agreement with the direct $m_{\mathrm{t}}$ measurements is a triumph of high precision experimentation coupled with theoretical high precision calculations at the quantum level of the Standard Model. Similarly, the mass of the charged weak boson, $m_{\mathrm{W}}$, was determined from precision measurements at the $\mathrm{Z}$ boson resonance and compared with direct measurements performed at the Tevatron and at high energies in a later phase of LEP operation.

But an important piece is still missing: the direct observation of the Higgs boson and the proof whether the electroweak symmetry breaking mechanism works as predicted by the Standard Model. The search for the Standard Model Higgs boson at the highest energies at LEP has yielded a lower bound on its mass, $m_{\mathrm{H}}>114 \mathrm{GeV}$ [7]. It is ongoing at the Tevatron and at the large hadron collider (LHC) that recently started the measurements of processes in protonproton collisions at the highest energies. However, the mass of the Higgs boson has been estimated considering it as a virtual particle emitted and reabsorbed by the electroweak bosons shifting slightly their masses. The combined results of LEP, SLD and the Tevatron put very strong constraints on the mass range allowed for a Standard Model Higgs boson, $m_{\mathrm{H}}<157 \mathrm{GeV}$. This implies that the Higgs boson or something equivalent must exist, and the mass should be well within the reach of the LHC. However, it could happen that the LHC will not find a Higgs boson. This could mean that the Higgs boson properties are rather different from that predicted 
in the Standard Model, or the Higgs boson does not exist, and definitely physics beyond the Standard Model would be necessary to explain this. But even if the Higgs boson will be found in the expected mass range, new phenomena beyond the Standard Model could be at work: since the Standard Model is fixed if the Higgs mass is known, also the Higgs couplings to electroweak bosons and fermions as well as the self-couplings are defined. If the measurement of the Higgs boson properties will not confirm these predictions, the mechanism of symmetry breaking is different from that suggested in the Standard Model. Thus, the question for the Higgs boson is crucial, also for the following reason: radiative corrections affect the mass of the Higgs boson and generate a shift which diverges quadratically with the cutoff energy. If one assumes that the Standard Model is correct up to the energy scale of the grand unification, the correction to the Higgs mass becomes the order of the grand unification scale itself. Within the Standard Model this infinity has to be neutralized requiring a fine-tuning of parameters. Although technically legitimate, this procedure is considered unnatural because it is a delicate cancellation between the fundamental quantity - the bare Higgs mass - and the quantum corrections to it. Theorists prefer more natural solutions, e.g. Supersymmetry, where the Higgs boson is connected with a new symmetry protecting the Higgs mass against divergences.

By now the Standard Model is in a good shape and describes sucessfully the experimental results, but new findings are eagerly awaited from experiments at the LHC.

This report gives an overview of the precision electroweak measurements at high energies which essentially determined the status of the Standard Model. It demonstrates how the Standard Model parameters have been derived by combining the variety of electroweak measurements at LEP and SLC with results from the Tevatron and confronting them with theoretical predictions. The conclusion-the consistency of the measurements and their agreement with the Standard Model up to the level of quantum corrections-is well known. But facing the physics findings expected at the LHC it will be very useful for further understanding and interpretation to recall the reader of the procedure used to establish the precision electroweak results.

The review is organized as follows: section 2 introduces the basics of the Standard Model. The choice of observables and their relation to the parameters of electroweak interactions is described in section 3. Experimental issues, such as the measurement of the luminosity, the beam energy and polarization are presented in section 4 . The variety of precision measurements at the $\mathrm{Z}$ pole is presented in section 5 , the properties of the $Z$ boson are summarized in section 6 . The Standard Model parameters derived in a global analysis are presented in section 7. The global analysis includes constraints from LEP-II measurements above the $\mathrm{Z}$ boson resonance and from measurements of the $\mathrm{W}$ boson and top quark masses at the Tevatron, and it compares with results from measurements at low energies. The combined data constrain the mass range for the Higgs boson. In section 8, LEP measurements at energies above the $\mathrm{Z}$ boson resonance are confronted with Standard Model predictions. Possible deviations could be a sign of new phenomena beyond the Standard Model. Several concepts for new physics are sketched in section 9. Some of these new models have been tested using the precision measurements at LEP and SLD. A popular model for physics beyond the Standard Model is chosen to demonstrate shortly the importance of precision measurements to reveal underlying new physics. A brief overall summary is given in section 10 .

\section{The Standard Model}

The Standard Model of particle physics describes the fundamental particles of the Universe and how they interact via the strong and electroweak forces. The fundamental particles include the fermions-quarks and leptons, which combine to form macroscopic objects-and the force particles, the bosons, which transmit interactions between the fermions. All Standard Model particles are considered as point-like.

\subsection{The building blocks of the Standard Model}

The Standard Model (SM) is defined by the GlashowWeinberg-Salam Model of electroweak interactions [1,2] together with the QCD sector of strong interactions [8]. It is based on the gauge group

$$
\begin{aligned}
G_{\mathrm{SM}} & =S U(3)_{\mathrm{C}} \times G_{\mathrm{GSW}} \\
& =S U(3)_{\mathrm{C}} \times S U(2)_{\mathrm{L}} \times U(1)_{\mathrm{Y}}
\end{aligned}
$$

of unitary gauge transformations. This means that physical equations are invariant under transformations applied independently at each space-time point. The $S U(3)_{\mathrm{C}}$ is a non-Abelian symmetry group of the strong interactions and distinguishes colour triplets of quarks and conjugate triplets of antiquarks leaving leptons as colour singlets. The fermions are classified by the quantum numbers of the weak isospin, $T, T_{3}$ and the weak hypercharge, $Y$. The representation of $S U(2)_{\mathrm{L}}$ are the weak isospin doublets of the left-handed fermions and the isospin singlets of right-handed fermions. The $U(1)_{Y}$ assigns the weak hypercharge, $Y$, to the $S U(2)_{\mathrm{L}}$ doublets and singlets. The relation between the quantum numbers and the electric charge is given by the Gell-Mann-Nishijima relation

$$
Q=T_{3}+\frac{Y}{2} \text {. }
$$

Three families of leptons and quarks exist:

$$
\begin{aligned}
& \left(\begin{array}{c}
v_{\mathrm{e}} \\
\mathrm{e}
\end{array}\right)_{\mathrm{L}}, \quad\left(\begin{array}{l}
\mathrm{u} \\
\mathrm{d}
\end{array}\right)_{\mathrm{L}}, \quad \mathrm{e}_{\mathrm{R}}, \mathrm{u}_{\mathrm{R}}, \mathrm{d}_{\mathrm{R}}, \\
& \left(\begin{array}{l}
v_{\mu} \\
\mu
\end{array}\right)_{\mathrm{L}}, \quad\left(\begin{array}{l}
\mathrm{c} \\
\mathrm{s}
\end{array}\right)_{\mathrm{L}}, \mu_{\mathrm{R}}, \mathrm{c}_{\mathrm{R}}, \mathrm{s}_{\mathrm{R}}, \\
& \left(\begin{array}{c}
v_{\tau} \\
\tau
\end{array}\right)_{L}, \quad\left(\begin{array}{l}
t \\
b
\end{array}\right)_{L}, \quad \tau_{R}, \quad t_{R}, \quad b_{R}
\end{aligned}
$$

complemented by right-handed neutrinos. The weak isospin, $T=\frac{1}{2}$, is assigned to doublets: up-type quarks (u,c,t) and neutrinos carry $T_{3}=+\frac{1}{2}$, down-type quarks $(\mathrm{d}, \mathrm{s}, \mathrm{b})$ and electron, muon and tau lepton carry $T_{3}=-\frac{1}{2}$. All singlets have the weak isospin $T=0, T_{3}=0$. Between fermions 
act electromagnetic, weak and strong forces associated with spin-1-fields, describing the photon $(\gamma)$, the electroweak gauge bosons $W^{+}, W^{-}$and $\mathrm{Z}$, and the gluon $\mathrm{g}$. The gauge invariance requires for each of the factors in $G_{\mathrm{SM}}$ (1) the presence of (originally) massless gauge fields coupling to the matter fields. The gauge fields for weak and electromagnetic interactions are denoted by $W_{\mu i},(i=1,2,3)$ and $B_{\mu}$ with the corresponding gauge couplings, $g$ and $g^{\prime}$. The physical gauge bosons are the photon,

$$
A_{\mu}=\cos \theta_{\mathrm{W}} B_{\mu}+\sin \theta_{\mathrm{W}} W_{\mu 3},
$$

the neutral weak boson

$$
Z_{\mu}=\cos \theta_{\mathrm{W}} W_{\mu 3}-\sin \theta_{\mathrm{W}} B_{\mu},
$$

and the charged weak bosons

$$
W_{\mu}^{ \pm}=\frac{1}{\sqrt{2}}\left(W_{\mu 1} \mp \mathrm{i} W_{\mu 2}\right)
$$

and $\sin \theta_{\mathrm{W}}$ is the weak mixing angle.

The couplings of the bosons $\gamma, \mathrm{Z}$ and $\mathrm{W}^{ \pm}$to leftand right-handed fermions are described by electromagnetic currents, $\mathcal{J}_{\mathrm{em}}^{\mu}$, neutral weak currents, $\mathcal{J}_{\mathrm{NC}}^{\mu}$, and charged weak currents, $\mathcal{J}_{\mathrm{CC}}^{\mu}$,

$$
\begin{aligned}
& \mathcal{J}_{\mathrm{em}}^{\mu}=\bar{\psi} \gamma^{\mu}(\boldsymbol{Q}) \psi \quad \text { with } \boldsymbol{Q}=\boldsymbol{T}_{3}+\frac{\boldsymbol{Y}}{2}, \\
& \mathcal{J}_{\mathrm{NC}}^{\mu}=\bar{\psi} \gamma^{\mu}\left(\boldsymbol{T}_{3}-2 \sin ^{2} \theta_{\mathrm{W}} \boldsymbol{Q}\right) \psi, \\
& \mathcal{J}_{\mathrm{CC}}^{\mu}=\bar{\psi} \gamma^{\mu}\left(\boldsymbol{T}_{1} \mp \mathrm{i} \boldsymbol{T}_{2}\right) \psi .
\end{aligned}
$$

The neutral current, $\mathcal{J}_{\mathrm{NC}}^{\mu}=\bar{\psi} \gamma^{\mu}\left(v_{\mathrm{f}}-a_{\mathrm{f}} \gamma_{5}\right) \psi$, conserves the fermion flavour and includes a vector coefficient, $v_{\mathrm{f}}=$ $T_{3}^{\mathrm{f}}-2 Q_{\mathrm{f}} \sin ^{2} \theta_{\mathrm{W}}$, and an axial-vector coefficient, $a_{\mathrm{f}}=T_{3}^{\mathrm{f}}$. The charged current has the general form

$$
\mathcal{J}_{\mathrm{CC}}^{\mu}=\sum_{\mathrm{f}} \bar{v} \gamma_{\mu}\left(1-\gamma_{5}\right) \ell+(\overline{\mathrm{u}}, \overline{\mathrm{c}}, \overline{\mathrm{t}}) \gamma_{\mu}\left(1-\gamma_{5}\right) V_{\mathrm{CKM}}\left(\begin{array}{c}
\mathrm{d} \\
\mathrm{s} \\
\mathrm{b}
\end{array}\right)
$$

and allows flavour changing through the unitary CabibboKobayashi-Maskawa matrix $V_{\mathrm{CKM}}$.

The Lagrangian for the interaction of matter fields has the form

$\mathcal{L}=-\frac{g}{2 \sqrt{2}}\left(J_{\mu}^{\mathrm{CC}} W^{\mu-}+\right.$ h.c. $)-\frac{g}{2 \cos \theta_{\mathrm{W}}} J_{\mu}^{\mathrm{NC}} Z^{\mu}-e \mathcal{J}_{\mu}^{\mathrm{em}} A^{\mu}$

with $\tan \theta_{\mathrm{W}}=g^{\prime} / g$ as ratio of the gauge couplings $g$ and $g^{\prime}$ and $e=g \sin \theta_{\mathrm{W}}$.

With the observation of neutral current interactions in neutrino-electron and neutrino-nucleon scattering in 1973 $[3,4]$ and the discovery of the $\mathrm{W}$ and $\mathrm{Z}$ bosons in protonantiproton collisions at CERN ten years later $[5,6]$, it was confirmed that $\mathrm{W}$ and $\mathrm{Z}$ bosons are massive. In the Standard Model the bosons (4), (5), originally massless, become massive by the Higgs mechanism: an additional scalar Higgs field couples to the gauge fields and to the fermions in a gauge invariant way. The Higgs field has a non-vanishing vacuum expectation value, $v$; the symmetry is 'broken'. Due to invariance under transformations in $S U(2)_{\mathrm{L}} \times U(1)_{Y}$, three of the four fields of the complex Higgs doublet are assigned to the fields of $\mathrm{W}$ and $\mathrm{Z}$ boson; the remnant field denotes the physical scalar Higgs boson. With the Higgs mechanism the relations between gauge couplings and particle masses are given. The gauge boson masses are

$$
m_{\mathrm{W}}=\frac{1}{2} g v, \quad m_{\mathrm{Z}}=\frac{1}{2} \frac{g v}{\cos \theta_{\mathrm{W}}},
$$

and the fermion masses and the Higgs boson mass are

$$
m_{\mathrm{f}}=\frac{G_{\mathrm{f}} v}{\sqrt{2}}, \quad m_{\mathrm{H}}=\sqrt{2 \lambda} v,
$$

with the Higgs boson self-coupling $\lambda$ and the Yukawa couplings $G_{\mathrm{f}}$ of fermions. So far the Higgs boson has not been observed directly. The discovery of the Higgs boson and the measurement of its mass, couplings to fermions and bosons and self-interaction, will verify whether the Higgs boson behaves as predicted by the mechanism of electroweak symmetry breaking in the Standard Model.

At low energies, $q^{2} \ll m_{\mathrm{W}}^{2}$, electroweak interactions are expressed by the current-current structure of point-like fourfermion interactions,

$$
\mathcal{L}_{\text {eff }}=-\frac{1}{\sqrt{2}}\left(G_{\mathrm{F}} J_{\mu}^{+} J^{\mu-}+G_{\mathrm{NC}} J_{\mu}^{\mathrm{Z}} J^{\mu \mathrm{Z}}\right)-e \mathcal{J}_{\mu}^{\mathrm{em}} A^{\mu} .
$$

The consistency of effective four-fermion interactions and interactions proceeding via the exchange of a massive weak boson requires for the charged current coupling

$$
\sqrt{2} G_{\mathrm{F}}=\frac{g^{2}}{4 m_{\mathrm{W}}^{2}}
$$

and the neutral current coupling corresponds to

$$
\sqrt{2} G_{\mathrm{NC}}=\frac{g^{2}}{4 m_{\mathrm{Z}}^{2} \cos ^{2} \theta_{\mathrm{W}}} .
$$

Originally, the ratio of neutral current to charged current couplings was used to define the $\rho$ parameter [12],

$$
\frac{G_{\mathrm{NC}}}{G_{\mathrm{CC}}}=\rho_{\text {tree }} .
$$

The $\rho$ parameter is determined by the Higgs structure of the theory, and nowadays it is usually defined as

$$
\rho_{\text {tree }}=\frac{m_{\mathrm{W}}^{2}}{m_{\mathrm{Z}}^{2} \cos ^{2} \theta_{\mathrm{W}}} \equiv 1,
$$

which is unity at tree-level for the minimal Standard Model containing only Higgs doublets. Using the relation $4 \pi \alpha=$ $e^{2}=g \sin ^{2} \theta_{\mathrm{W}}$, the Fermi constant $G_{\mathrm{F}}$ can be expressed as

$$
\sqrt{2} G_{\mathrm{F}} m_{\mathrm{W}}^{2}\left(1-\frac{m_{\mathrm{Z}}^{2}}{m_{\mathrm{W}}^{2}}\right)=\sqrt{2} G_{\mathrm{F}} m_{\mathrm{W}}^{2} \sin ^{2} \theta_{\mathrm{W}}=\pi \alpha .
$$

$G_{\mathrm{F}}$ is measured in $\mu$ decay, $G_{\mathrm{F}}=1.16637(1) \times$ $10^{-5}(\mathrm{GeV})^{-2}$ [9]. Due to the Standard Model relation

$$
G_{\mathrm{F}}=\frac{g^{2}}{4 \sqrt{2} m_{\mathrm{W}}^{2}}=\frac{1}{\sqrt{2} v^{2}},
$$


the vacuum expectation value $v$ is very precisely known and figures as a conversion factor between masses and couplings. It is also called the 'Fermi scale'. As a consequence of equation (16) large particle masses require strong couplings, very heavy particle masses compared with the Fermi scale are unnatural in the framework of this minimal Standard Model.

The couplings between $\mathrm{Z}$ boson and fermions depend on the weak isospin, $T_{3}$, the electric charge and the weak mixing angle, and comprise a vector and axial-vector contribution,

$$
\begin{gathered}
g_{\mathrm{V}} \equiv T_{3}^{\mathrm{f}}-2 Q_{\mathrm{f}} \sin ^{2} \theta_{\mathrm{W}}, \\
g_{\mathrm{A}} \equiv T_{3}^{\mathrm{f}},
\end{gathered}
$$

or, equivalently expressed by left- and right-handed couplings,

$$
g_{\mathrm{L}} \equiv \frac{g_{\mathrm{V}}+g_{\mathrm{A}}}{2}, \quad g_{\mathrm{R}} \equiv \frac{g_{\mathrm{V}}-g_{\mathrm{A}}}{2}
$$

The measurement of the couplings reveals the weak mixing angle and gives information about the Higgs structure of the Standard Model. The universal coupling of leptons to the $\mathrm{Z}$ boson is inherent to the Standard Model, and its test includes necessarily the test of lepton universality.

\subsection{Radiative corrections}

The tree-level approximation is not sufficient to calculate physical processes at the level of precision required by the experiments described here. Radiative corrections-a main feature in quantum field theories-have to be included. In quantum field theories a physical process is described by taking into account all possible Feynman graphs in perturbation theory, including loop diagrams. This yields power series in coupling constants. Possible divergences from loop diagrams are cancelled by renormalization constants. In practice, loworder perturbation theory is used, and the results of perturbative calculations are expressed in terms of precisely known physical quantities. In the electroweak theory, one works usually in the on-mass-shell renormalization scheme (OMS) [10] and considers the electromagnetic coupling constant as scale dependent, i.e. it runs with the energy. The boson and fermion masses are fixed at their measured values, and some of the tree-level relations are preserved, in particular the expression for the weak mixing angle, $\sin ^{2} \theta_{\mathrm{W}}$. This introduces energy dependences of the vector and axial-vector coupling constants; they also become scale dependent. Including one-loop radiative corrections, one distinguishes between fermion and boson loop insertions in propagators (self-energy corrections) as shown in figure 1, flavour dependent vertex corrections as depicted in figure 2 for the $\mathrm{Zb} \bar{b}$ vertex representing the most important vertex corrections, and corrections from box diagrams. The radiative corrections modify the tree-level relation (15), and it becomes

$$
\sqrt{2} G_{\mathrm{F}} m_{\mathrm{W}}^{2}\left(1-\frac{m_{\mathrm{W}}^{2}}{m_{\mathrm{Z}}^{2}}\right)=\frac{\pi \alpha(0)}{1-\Delta r},
$$
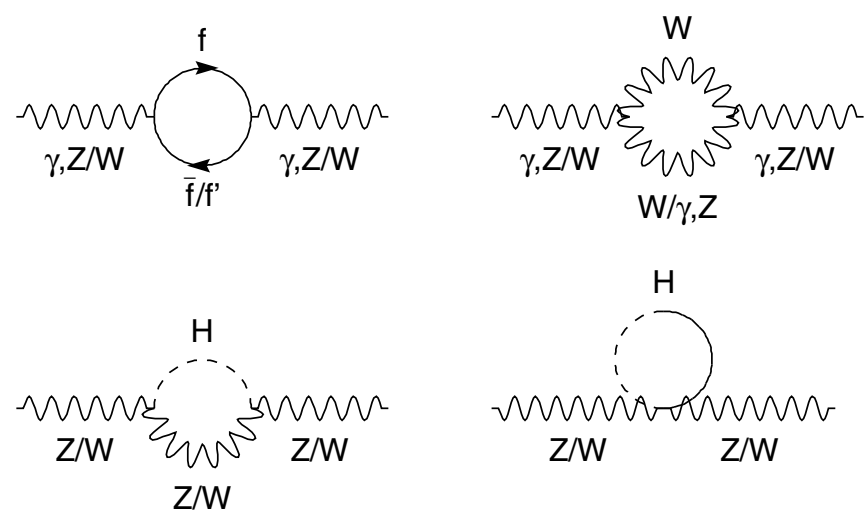

Figure 1. Higher-order corrections to the gauge bosons due to boson and fermion loops.
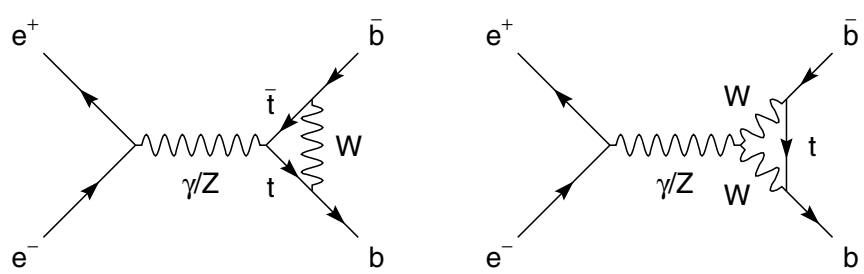

Figure 2. Vertex corrections to the process $\mathrm{e}^{+} \mathrm{e}^{-} \rightarrow \mathrm{b} \overline{\mathrm{b}}$.

defining the charged current variable $\Delta r$. In the on-massshell renormalization scheme the weak mixing angle is defined uniquely through the gauge boson masses,

$$
\sin ^{2} \theta_{\mathrm{W}} \equiv 1-\frac{m_{\mathrm{W}}^{2}}{m_{\mathrm{Z}}^{2}} .
$$

At high momentum transfer, i.e. at the $\mathrm{Z}$ pole, this fundamental form is preserved using the effective weak mixing angle,

$$
\cos ^{2} \theta_{\text {eff }}^{\mathrm{f}} \sin ^{2} \theta_{\text {eff }}^{\mathrm{f}}=\frac{\pi \alpha(0)}{\sqrt{2} m_{\mathrm{Z}}^{2} G_{\mathrm{F}}} \frac{1}{1-\Delta r^{\mathrm{f}}},
$$

where $\Delta r^{\mathrm{f}}$ derives from the weak neutral current and the index $\mathrm{f}$ denotes the flavour dependence of the corrections. The radiative corrections are parametrized for both, low momentum transfer and at the $\mathrm{Z}$ pole,

$$
\begin{gathered}
\Delta r=\Delta \alpha+\Delta r_{\mathrm{w}}, \\
\Delta r^{\mathrm{f}}=\Delta \alpha+\Delta r_{\mathrm{w}}^{\mathrm{f}},
\end{gathered}
$$

where $\Delta \alpha$ is the correction due to fermion loops in the photon propagator. The large change in scale from zero momentum to high energies yields a large correction $\Delta \alpha$. The weak parts, $\Delta r_{\mathrm{w}}$ and $\Delta r_{\mathrm{w}}^{\mathrm{f}}$, specify the corrections due to weak bosons $(\mathrm{Z}, \mathrm{W}, \mathrm{H})$. They are important quantities predicted by the Standard Model, and are sensitive to the underlying physics. Therefore, they contain information on the masses of the weak bosons and the top quark.

The running electromagnetic coupling. The fine structure constant $\alpha$ is known with the impressive accuracy of $7 \times 10^{-10}$ at $q^{2} \simeq 0$. However, due to quantum corrections it is running 
with the energy. Fermion loop insertions into the photon propagator as shown in figure 1 increase the electromagnetic coupling from the zero $q^{2}$ limit, $\alpha=1 / 137.036$, to $1 / 128.945$ at energies $\sqrt{s} \approx m_{\mathrm{Z}}$. This effect is also known as vacuum polarization. The running of the fine structure constant has to be taken into account to describe Standard Model processes at high energies precisely. The corresponding correction, $\Delta \alpha$, is

$$
\alpha(s)=\frac{\alpha(0)}{1-\Delta \alpha(s)}
$$

with

$$
\Delta \alpha(s)=\Delta \alpha_{\text {lept }}(s)+\Delta \alpha_{\text {top }}(s)+\Delta \alpha_{\text {had }}^{(5)}(s) .
$$

At $s=m_{\mathrm{Z}}^{2}$ the leptonic contribution, $\Delta \alpha_{\text {lept }}(s)$, can be precisely calculated

$$
\Delta \alpha_{\text {lept }}=\frac{\alpha(0)}{3 \pi} \sum_{\mathrm{e}, \mu, \tau}\left(\log \frac{m_{\mathrm{Z}}^{2}}{m_{\ell}^{2}}-\frac{5}{3}\right)+\mathcal{O} \frac{m_{\ell}^{2}}{m_{\mathrm{Z}}^{2}},
$$

and is known to third order. The top quark contribution, $\Delta \alpha_{\text {top }}$, is small and well known if $m_{\mathrm{t}}$ is specified. However, for the light hadronic part, $\Delta \alpha_{\text {had }}^{(5)}(s)$, perturbative QCD is not applicable and quark masses are not reasonable input parameters. This term can be calculated within certain theoretical models or using also experimental data. The evaluation of the hadronic contribution to the vacuum polarization, $\Delta \alpha_{\mathrm{had}}^{(5)}\left(m_{\mathrm{Z}}^{2}\right)$, is important for the analysis of precision data. Its treatment in the global electroweak analysis is discussed in section 7 .

Weak corrections in $\Delta r$. The weak parts, $\Delta r_{\mathrm{w}}$ and $\Delta r_{\mathrm{w}}^{\mathrm{f}}$, in the corrections to $\Delta r$ and $\Delta r^{\mathrm{f}}$ contain the parameter $\Delta \rho$, plus a remainder $[10,11]$,

$$
\begin{gathered}
\Delta r_{\mathrm{w}}=-\frac{\cos ^{2} \theta_{\mathrm{W}}}{\sin ^{2} \theta_{\mathrm{W}}} \Delta \rho+\cdots, \\
\Delta r_{\mathrm{w}}^{\mathrm{f}}=-\Delta \rho+\cdots,
\end{gathered}
$$

where $\Delta \rho$ is the weak correction to the $\rho$ parameter (14),

$$
\rho=1+\Delta \rho \text {. }
$$

The bulk of the electroweak corrections to the couplings at the $\mathrm{Z}$ pole is absorbed into complex form factors, $\mathcal{R}_{\mathrm{f}}$ for the overall scale, and $\mathcal{K}_{\mathrm{f}}$ for the on-shell electroweak mixing angle $\sin ^{2} \theta_{\mathrm{W}}[12,13]$. As a result, the tree-level couplings (17) and (18) are modified to complex effective couplings:

$$
\begin{gathered}
\mathcal{G}_{\mathrm{Vf}}=\sqrt{\mathcal{R}_{\mathrm{f}}}\left(T_{3}^{\mathrm{f}}-2 Q_{\mathrm{f}} \mathcal{K}_{\mathrm{f}} \sin ^{2} \theta_{\mathrm{W}}\right) \\
\mathcal{G}_{\mathrm{Af}}=\sqrt{\mathcal{R}_{\mathrm{f}}} T_{3}^{\mathrm{f}} .
\end{gathered}
$$

In terms of the real parts of the complex form factors,

$$
\begin{gathered}
\rho_{\mathrm{f}} \equiv \operatorname{Re}\left(\mathcal{R}_{\mathrm{f}}\right)=1+\Delta \rho_{\mathrm{se}}+\Delta \rho_{\mathrm{f}}, \\
\kappa_{\mathrm{f}} \equiv \operatorname{Re}\left(\mathcal{K}_{\mathrm{f}}\right)=1+\Delta \kappa_{\mathrm{se}}+\Delta \kappa_{\mathrm{f}},
\end{gathered}
$$

the real effective couplings and the effective electroweak mixing angle are defined as

$$
\begin{gathered}
\sin ^{2} \theta_{\text {eff }}^{\mathrm{f}} \equiv \kappa_{\mathrm{f}} \sin ^{2} \theta_{\mathrm{W}} \\
g_{\mathrm{Vf}} \equiv \sqrt{\rho_{\mathrm{f}}}\left(T_{3}^{\mathrm{f}}-2 Q_{\mathrm{f}} \sin ^{2} \theta_{\mathrm{eff}}^{\mathrm{f}}\right) \\
g_{\text {Af }} \equiv \sqrt{\rho_{\mathrm{f}}} T_{3}^{\mathrm{f}},
\end{gathered}
$$

so that

$$
\frac{g_{\mathrm{Vf}}}{g_{\mathrm{Af}}}=\operatorname{Re}\left(\frac{\mathcal{G}_{\mathrm{Vf}}}{\mathcal{G}_{\mathrm{Af}}}\right)=1-4\left|Q_{\mathrm{f}}\right| \sin ^{2} \theta_{\mathrm{eff}}^{\mathrm{f}} .
$$

The quantities $\Delta \rho_{\mathrm{se}}$ and $\Delta \kappa_{\mathrm{se}}$ are universal corrections arising from the propagator self-energies, while $\Delta \rho_{\mathrm{f}}$ and $\Delta \kappa_{\mathrm{f}}$ are flavour specific vertex corrections. The leading order terms in $\Delta \rho_{\mathrm{se}}$ and $\Delta \kappa_{\mathrm{se}}$ depend quadratically on the top mass,

$$
\begin{gathered}
\Delta \rho_{\mathrm{se}} \propto \frac{m_{\mathrm{t}}^{2}}{m_{\mathrm{W}}^{2}}+\cdots, \\
\Delta \kappa_{\mathrm{se}} \propto \frac{m_{\mathrm{t}}^{2}}{m_{\mathrm{W}}^{2}} \frac{\cos ^{2} \theta_{\mathrm{W}}}{\sin ^{2} \theta_{\mathrm{W}}}+\cdots,
\end{gathered}
$$

but only logarithmically on the Higgs boson mass,

$$
\Delta \rho_{\mathrm{se}}, \Delta \kappa_{\mathrm{se}} \propto \ln \frac{m_{\mathrm{H}}^{2}}{m_{\mathrm{W}}^{2}} .
$$

The flavour dependence is very small for all fermions except the b-quarks. The corrections to the $b \bar{b}$ vertex result in significant additional contributions from tWb couplings (see figure 2) due to the large mass splitting between bottom and top quark and the size of the CKM matrix element $\left|V_{\mathrm{tb}}\right| \simeq 1$,

$$
\begin{aligned}
\Delta \kappa_{\mathrm{b}} & =\frac{G_{\mathrm{F}} m_{\mathrm{t}}^{2}}{4 \sqrt{2} \pi^{2}}+\cdots, \\
\Delta \rho_{\mathrm{b}} & =-2 \Delta \kappa_{\mathrm{b}}+\cdots
\end{aligned}
$$

Details of these corrections can be found in [14-23].

The discussion of radiative corrections given here is far from being complete. The actual calculations employed in the analyses are performed to higher order, using the programs ZFITTER [23] and TOPAZ0 [24]. These programs have been created for precision analyses of fermion-pair production processes at LEP and SLC. They include electroweak corrections, photonic corrections and QCD corrections. The theoretical precision of predictions for the Standard Model are below the per-mille level, the electroweak corrections itself amount approximately to the (few) per cent level. Thus, the analysis of electroweak observables is sensitive to the loop corrections. Details of the calculation of radiative corrections can be found in the program descriptions and the references therein. The interested reader is encouraged to consult also the authoritative discussion in [25]. 


\subsection{Input parameters for Standard Model analyses}

The interactions and particle properties are fixed by the parameters of the Standard Model. These are the fermion masses, the QCD and electroweak gauge couplings $g_{\mathrm{s}}, g$ and $g^{\prime}$, and the Higgs boson couplings. Usually, relations are written in terms of parameters that can be measured directly. Due to the relations between masses and couplings these parameters are not independent and different parametrizations are possible. The specific choice of input parameters defines the parametrization (also called renormalization scheme). The on-mass-shell scheme is a natural choice and based on the fine structure constant $\alpha$ and the physical particle masses:

$$
\alpha, m_{\mathrm{W}}, m_{\mathrm{Z}}, m_{\mathrm{f}}, m_{\mathrm{H}}, \alpha_{\mathrm{S}} .
$$

The strong coupling constant, $\alpha_{\mathrm{S}}$ comprises QCD corrections. The weak coupling constants, $\mathcal{G}_{\mathrm{Af}}$ and $\mathcal{G}_{\mathrm{Vf}}$, are determined by the quantum numbers, the boson masses and radiative corrections which are also given by fermion and boson masses. The $\mathrm{W}$ boson mass is much less precisely known than the $\mathrm{Z}$ boson mass measured at the $\mathrm{Z}$ boson resonance. However, the 9 ppm precision on the Fermi constant determined in muon decay is much higher than the relative precision with which $m_{\mathrm{W}}$ can be measured in the foreseeable future. Using relation (20) between $m_{\mathrm{W}}, m_{\mathrm{Z}}$ and $G_{\mathrm{F}}$,

$$
m_{\mathrm{W}}^{2}=\frac{m_{\mathrm{Z}}^{2}}{2}\left(1+\sqrt{1-4 \frac{\pi \alpha}{\sqrt{2} G_{\mathrm{F}} m_{\mathrm{Z}}^{2}} \frac{1}{1-\Delta r}}\right)
$$

the direct dependence on the $\mathrm{W}$ boson mass can be eliminated and replaced by the Fermi constant $G_{\mathrm{F}}$ which is treated as a constant in the analyses. Thus, the Standard Model input parameters are

$$
\alpha, G_{\mathrm{F}}, m_{\mathrm{Z}}, m_{\mathrm{f}}, m_{\mathrm{H}}, \alpha_{\mathrm{S}}
$$

Among the fermion masses the top mass, $m_{\mathrm{t}}$, is of special interest. The substitution (45) introduces further significant dependence on $m_{\mathrm{t}}$ and $m_{\mathrm{H}}$ through $\Delta r$. Hence it was possible to determine $m_{\mathrm{t}}$ well before the discovery of the top quark by analysing precision measurements performed at the $\mathrm{Z}$ pole. The consistency of the indirectly determined $\mathrm{W}$ boson mass and its direct measurement is another powerful test of the Standard Model.

\section{Precision observables}

The production of fermion-pairs in electron-positron collisions is mediated by neutral gauge bosons. At centreof-mass energies around $m_{\mathrm{Z}}$, the so-called $\mathrm{Z}$ resonance, this process is determined by the exchange of $Z$ bosons. Based on the measurements of the $\mathrm{Z}$ resonance, the $\mathrm{Z}$ boson properties are identified and the Standard Model is tested. The choice of observables which is most sensitive to the $\mathrm{Z}$ boson parameters is presented in the following section.
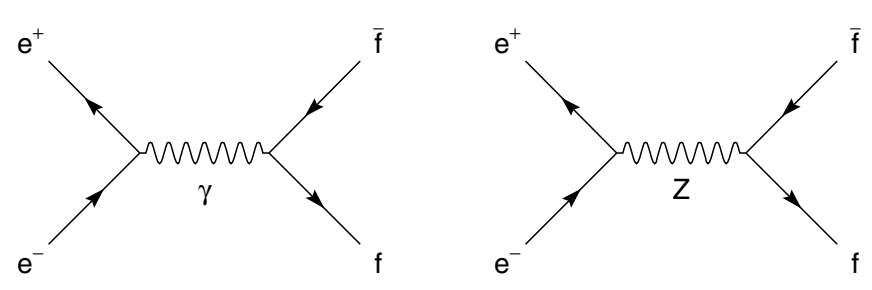

Figure 3. Feynman diagrams in lowest order for the process $\mathrm{e}^{+} \mathrm{e}^{-} \rightarrow \mathrm{f} \overline{\mathrm{f}}$.

\subsection{Fermion-pair cross section}

The annihilation of electrons and positrons to fermion-pairs, $\mathrm{e}^{+} \mathrm{e}^{-} \rightarrow \mathrm{f} \overline{\mathrm{f}}$, proceeds via virtual photon and $\mathrm{Z}$ boson exchange in the $s$-channel as shown in figure 3 for the lowest order Feynman diagrams. The corresponding matrix element includes contributions from photon and $\mathrm{Z}$ boson exchange, $\mathcal{M}=\mathcal{M}_{\gamma}+\mathcal{M}_{\mathrm{Z}}$, and the cross section includes contributions from pure photon and pure $\mathrm{Z}$ boson exchange and the $\gamma-\mathrm{Z}$ interference term. Taking into account the helicity and angular momentum conservation, the amplitudes are

$$
\begin{aligned}
& \mathcal{M}\left(\mathrm{e}_{i}^{-} \mathrm{e}_{j}^{+} \rightarrow \mathrm{f}_{i} \overline{\mathrm{f}}_{j}\right) \propto A_{i i}(1+\cos \theta), \\
& \mathcal{M}\left(\mathrm{e}_{i}^{-} \mathrm{e}_{j}^{+} \rightarrow \mathrm{f}_{j} \overline{\mathrm{f}}_{i}\right) \propto A_{i j}(1-\cos \theta),
\end{aligned}
$$

with

$$
A_{\mathrm{ik}}=Q_{\mathrm{f}} Q_{\mathrm{e}}+\frac{g_{i}^{\mathrm{e}} g_{k}^{\mathrm{f}}}{\cos \theta_{\mathrm{W}} \sin \theta_{\mathrm{W}}} \frac{s}{s-m_{\mathrm{Z}}^{2}+\mathrm{i} m_{\mathrm{Z}} \Gamma_{\mathrm{Z}}}
$$

and $i, k=\mathrm{L}, \mathrm{R}$. At energies close to the mass of the $\mathrm{Z}$ boson the resonant production of $\mathrm{Z}$ bosons dominates and affords the detailed study of $\mathrm{Z}$ boson parameters and the structure of the Standard Model. The cross section is given by the sum of the squared amplitudes, $\sigma \sim \sum_{i, k=\mathrm{L}, \mathrm{R}}\left|\mathcal{M}_{i k}\right|^{2}$. Taking into account the electroweak corrections and neglecting the radiation of photons off the initial and final states, the fermion masses and also the gluon radiation off final quarks, the differential cross section for the $s$-channel can be written for unpolarized beams as

$$
\frac{1}{N_{\mathrm{C}}^{\mathrm{f}}} \frac{2 s}{\pi} \frac{\mathrm{d} \sigma_{\mathrm{ew}}}{\mathrm{d} \cos \theta}(s, \cos \theta)_{\mathrm{ew}}=\sigma_{\gamma}+\sigma_{\gamma \mathrm{Z}}+\sigma_{\mathrm{Z}}
$$

with

$$
\begin{aligned}
\sigma_{\gamma}=\mid & \left.\alpha(s) Q_{\mathrm{f}}\right|^{2}\left(1+\cos ^{2} \theta\right), \\
\sigma_{\gamma \mathrm{Z}}= & -8 \operatorname{Re}\left\{\alpha ^ { \star } ( s ) Q _ { \mathrm { f } } \chi ( s ) \left[\mathcal{G}_{\mathrm{Ve}} \mathcal{G}_{\mathrm{Vf}}\left(1+\cos ^{2} \theta\right)\right.\right. \\
& \left.\left.+2 \mathcal{G}_{\mathrm{Ae}} \mathcal{G}_{\mathrm{Af}} \cos \theta\right]\right\} \\
\sigma_{\mathrm{Z}}= & +16|\chi(s)|^{2}\left[\left(\left|\mathcal{G}_{\mathrm{Ve}}\right|^{2}+\left|\mathcal{G}_{\mathrm{Ae}}\right|^{2}\right)\left(\left|\mathcal{G}_{\mathrm{Vf}}\right|^{2}+\left|\mathcal{G}_{\mathrm{Af}}\right|^{2}\right)\right. \\
& \left.\times\left(1+\cos ^{2} \theta\right)+8 \operatorname{Re}\left\{\mathcal{G}_{\mathrm{Ve}} \mathcal{G}_{\mathrm{Ae}}^{\star}\right\} \operatorname{Re}\left\{\mathcal{G}_{\mathrm{Vf}} \mathcal{G}_{\mathrm{Af}}^{\star}\right\} \cos \theta\right],
\end{aligned}
$$

where $\chi$ is the propagator,

$$
\chi(s)=\frac{G_{\mathrm{F}} m_{\mathrm{Z}}^{2}}{8 \pi \sqrt{2}} \frac{s}{s-m_{\mathrm{Z}}^{2}+\mathrm{i} s \Gamma_{\mathrm{Z}}(s) / m_{\mathrm{Z}}}
$$

and $\theta$ the scattering angle of the outgoing fermion with respect to the $\mathrm{e}^{-}$direction. The colour factor, $N_{\mathrm{C}}^{\mathrm{f}}$, is one for leptons 
and three for quarks. The resonance shape of the cross section at $\sqrt{s} \approx m_{\mathrm{Z}}$ is also called the $Z$ lineshape. The $s$-dependent $\mathrm{Z}$ boson width, $\Gamma_{\mathrm{Z}}(s)$, in the Breit-Wigner denominator of the propagator $(53)$, is one of the possible definitions $[15,26]$ and suggested as a result of quantum corrections.

In Bhabha scattering, $\mathrm{e}^{+} \mathrm{e}^{-} \rightarrow \mathrm{e}^{+} \mathrm{e}^{-}$, also $t$-channel diagrams of $\gamma$ and $\mathrm{Z}$ boson exchange exist, and interferences of $s$ - and $t$-channel diagrams add to the cross section given in equations (49)-(52). The $t$-channel contribution at low scattering angles $\theta$ (forward direction) is dominated by photon exchange and described by QED. The analysis of low-angle Bhabha scattering is used to measure the luminosity with high precision (see section 4). If one or both beams are polarized, the differential cross section depends also on the degree of polarization of electron and positron beam, $\mathcal{P}_{\mathrm{e}^{-}}$and $\mathcal{P}_{\mathrm{e}^{+}}$. For longitudinally polarized beams one finds

$$
\begin{aligned}
\frac{1}{N_{\mathrm{C}}^{\mathrm{f}}} & \frac{2 s}{\pi} \frac{\mathrm{d} \sigma_{\mathrm{ew}}^{\mathrm{pol}}}{\mathrm{d} \cos \theta}(s, \cos \theta)=\left(1-\mathcal{P}_{\mathrm{e}^{+}} \mathcal{P}_{\mathrm{e}^{-}}\right) \sigma_{\mathrm{ew}} \\
& +\left(\mathcal{P}_{\mathrm{e}^{+}}-\mathcal{P}_{\mathrm{e}^{-}}\right)\left(\sigma_{\gamma \mathrm{Z}}^{\mathrm{pol}}+\sigma_{\mathrm{Z}}^{\mathrm{pol}}\right),
\end{aligned}
$$

where $\sigma_{\mathrm{ew}}$ is the unpolarized cross section given in equation (49). The polarized contributions are

$$
\begin{aligned}
\sigma_{\gamma \mathrm{Z}}^{\mathrm{pol}}= & 8 \operatorname{Re}\left\{\alpha ^ { \star } ( s ) Q _ { \mathrm { f } } \chi ( s ) \left[\mathcal{G}_{\mathrm{Ae}} \mathcal{G}_{\mathrm{Vf}}^{\star}\left(1+\cos ^{2} \theta\right)\right.\right. \\
& \left.\left.+2 \mathcal{G}_{\mathrm{Ve}} \mathcal{G}_{\mathrm{Af}}^{\star} \cos \theta\right]\right\},
\end{aligned}
$$

$\sigma_{\mathrm{Z}}^{\mathrm{pol}}=-16|\chi(s)|^{2}\left\{2 \operatorname{Re}\left(\mathcal{G}_{\mathrm{Ve}} \mathcal{G}_{\mathrm{Ae}}^{\star}\right)\left[\left|\mathcal{G}_{\mathrm{Vf}}\right|^{2}+\left|\mathcal{G}_{\mathrm{Af}}\right|^{2}\right]\left(1+\cos ^{2} \theta\right)\right.$

$$
\left.+2 \operatorname{Re}\left(\mathcal{G}_{\mathrm{Vf}} \mathcal{G}_{\mathrm{Af}}^{\star}\right)\left[\left|\mathcal{G}_{\mathrm{Ve}}\right|^{2}+\left|\mathcal{G}_{\mathrm{Ae}}\right|^{2}\right] 2 \cos \theta\right\}
$$

The '-' sign of $\mathcal{P}_{\mathrm{e}^{-}}$describes a left-handed beam, ' + ' a right-handed beam. So far only the electron beam has been polarized at high-energy $\mathrm{e}^{+} \mathrm{e}^{-}$machines, the positrons have been unpolarized. Hence, for further considerations in this paper the electron polarization, $\mathcal{P}_{\mathrm{e}}$, is sufficient to define leftand right-handed cross sections, $\sigma_{\mathrm{L}}$ and $\sigma_{\mathrm{R}}$ :

$\frac{\mathrm{d} \sigma_{\mathrm{L}}}{\mathrm{d} \cos \theta}=\frac{\mathrm{d} \sigma^{\mathrm{pol}}}{\mathrm{d} \cos \theta}\left(\mathcal{P}_{\mathrm{e}}=-1\right), \quad \frac{\mathrm{d} \sigma_{\mathrm{R}}}{\mathrm{d} \cos \theta}=\frac{\mathrm{d} \sigma^{\mathrm{pol}}}{\mathrm{d} \cos \theta}\left(\mathcal{P}_{\mathrm{e}}=+1\right)$.

\subsection{Photon radiation}

Photon radiation from initial and final states, and their interference modify the cross sections (49) and (54). The most important diagrams are shown in figure 4. The dominating contribution arises from a photon radiated off the initial state yielding a reduced effective centre-of-mass energy for the kernel process. These photonic corrections are large, $(\mathcal{O}(30 \%))$, and they depend on experimental cuts. The photon radiation from initial and final states, and their interferences are treated by the convolution of the kernel cross section, $\sigma_{\mathrm{ew}}$, and the QED radiator function, $H_{\mathrm{QED}}$,

$$
\sigma(s)=\int_{4 m_{\mathrm{f}}^{2} / s}^{1} \mathrm{~d} z H_{\mathrm{QED}}(z, s) \sigma_{\mathrm{ew}}(z s) ;
$$

the parameter $z$ describes the reduced centre-of-mass energy for the kernel process. The radiator function $H_{\mathrm{QED}}$ is calculated within the framework of QED for total cross sections, forwardbackward and left-right cross sections. At $\sqrt{s}=m_{\mathrm{Z}}$, the
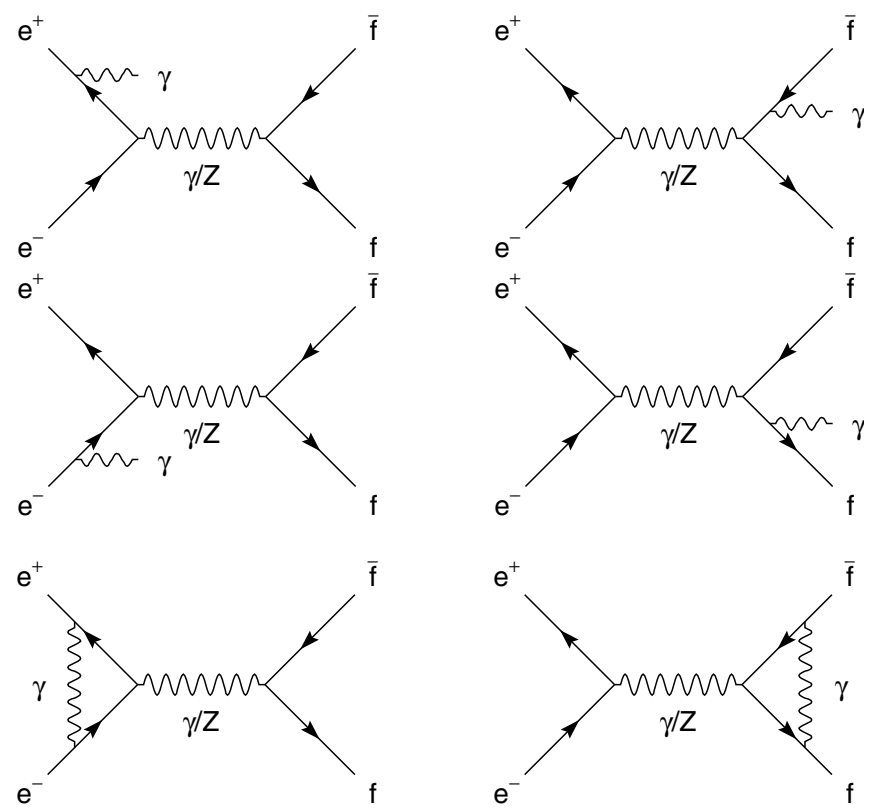

Figure 4. Some lowest order QED corrections to the process $\mathrm{e}^{+} \mathrm{e}^{-} \rightarrow \mathrm{f} \overline{\mathrm{f}}$. Together with photonic box diagrams which give much smaller contributions, they form a gauge invariant sub-set included in the radiator function $H_{\mathrm{QED}}$.

measured total cross section is not only substantially smaller than the QED-deconvoluted cross section, also the peak position is slightly shifted by about $+100 \mathrm{MeV}$. The $s$-channel QED-deconvoluted forward-backward asymmetries are offset on and below the $\mathrm{Z}$ peak by +0.017 . The photonic corrections to the initial and final state as well as the interference of them are implemented in the analysis programs ZFITTER [23] and TOPAZ0 [24]. Thanks to the effort made during the running of LEP, an excellent theoretical precision better than $0.1 \%$ has been achieved.

\subsection{Z boson widths}

In the approach used here, the $\mathrm{Z}$ boson width is predicted as a result of quantum corrections in the Standard Model and is thus naturally $s$-dependent. This is reflected in the definition of the mass and $s$-dependent width term in the Breit-Wigner denominator in equation (53). Another commonly used width definition, where the propagator takes the form $\chi(s) \propto$ $s /\left(s-\bar{m}_{Z}{ }^{2}+\mathrm{i} \bar{m}_{Z} \bar{\Gamma}_{Z}\right)$, yields equivalent $Z$ resonance shapes employing the transformations $\bar{m}_{\mathrm{Z}}=m_{\mathrm{Z}} / \sqrt{1+\Gamma_{\mathrm{Z}}^{2} / m_{\mathrm{Z}}^{2}}$ and $\bar{\Gamma}_{\mathrm{Z}}=\Gamma_{\mathrm{Z}} / \sqrt{1+\Gamma_{\mathrm{Z}}^{2} / m_{\mathrm{Z}}^{2}}$.

The $\mathrm{Z}$ boson partial widths depend on the sum of $\mathrm{Z}$ boson vector and axial-vector couplings squared and they are defined inclusively, i.e. they contain corrections due to photon and gluon emission from the final state [15, 19, 27-29],

$$
\Gamma_{\mathrm{f} \overline{\mathrm{f}}}=N_{\mathrm{C}}^{\mathrm{f}} \frac{G_{\mathrm{F}} m_{\mathrm{Z}}^{3}}{6 \pi \sqrt{2}}\left(\left|\mathcal{G}_{\mathrm{Vf}}\right|^{2} R_{\mathrm{Vf}}+\left|\mathcal{G}_{\mathrm{Af}}\right|^{2} R_{\mathrm{Af}}\right)+\Delta_{\mathrm{ew} / \mathrm{QCD}} .
$$

The term $\Delta_{\text {ew/QCD }}$ accounts for the non-factorizable electroweak and QCD corrections to the $\mathrm{Z}$ boson decay into quarks, the radiator functions, $R_{\mathrm{Af}}$ and $R_{\mathrm{Vf}}$, take into account 
final state QED and QCD corrections as well as contributions of non-zero fermion masses. To first order and for zero fermion masses the radiator functions are the same for vector and axialvector coupling,

$$
R_{\mathrm{A}, \mathrm{QCD}}=R_{\mathrm{V}, \mathrm{QCD}}=R_{\mathrm{QCD}}=1+\frac{\alpha_{\mathrm{S}}\left(m_{\mathrm{Z}}^{2}\right)}{\pi}+\cdots,
$$

for the QCD corrections to the final state quarks, and formally similar but much smaller due to the smaller electromagnetic coupling constant,

$$
R_{\mathrm{A}, \mathrm{QED}}=R_{\mathrm{V}, \mathrm{QED}}=R_{\mathrm{QED}}=1+\frac{3}{4} Q_{\mathrm{f}}^{2} \frac{\alpha\left(m_{\mathrm{Z}}^{2}\right)}{\pi}+\cdots,
$$

for the QED corrections to final state leptons and quarks. The partial widths add up to the total $\mathrm{Z}$ boson width,

$$
\Gamma_{\mathrm{Z}}=\sum_{f \neq t} \Gamma_{\mathrm{f} \overline{\mathrm{f}}}=\Gamma_{\mathrm{had}}+\Gamma_{\mathrm{ee}}+\Gamma_{\mu \mu}+\Gamma_{\tau \tau}+3 \Gamma_{\nu \bar{v}}
$$

where $\Gamma_{\text {had }}$ is the inclusive hadronic decay width summed over the five light quark flavours, u,d,s,c,b (the top quark is too heavy to be created in $\mathrm{Z}$ boson decays).

Considering only the $\mathrm{Z}$ boson exchange, the total cross section can be expressed by the $\mathrm{Z}$ boson decay widths to the initial and final states, $\Gamma_{\mathrm{ee}}$ and $\Gamma_{\mathrm{ff}}$;

$$
\sigma_{\mathrm{f} \overline{\mathrm{f}}}^{\mathrm{Z}}=\sigma_{\mathrm{f} \overline{\mathrm{f}}}^{\mathrm{peak}} \frac{s \Gamma_{\mathrm{Z}}^{2}}{\left(s-m_{\mathrm{Z}}^{2}\right)^{2}+s^{2} \Gamma_{\mathrm{Z}}^{2} / m_{\mathrm{Z}}^{2}},
$$

where

$$
\sigma_{\mathrm{f} \overline{\mathrm{f}}}^{\text {peak }}=\frac{1}{R_{\mathrm{QED}}} \sigma_{\mathrm{f} \overline{\mathrm{f}}}^{0}=\frac{1}{R_{\mathrm{QED}}} \frac{12 \pi}{m_{\mathrm{Z}}^{2}} \frac{\Gamma_{\mathrm{ee}} \Gamma_{\mathrm{f} \overline{\mathrm{f}}}}{\Gamma_{\mathrm{Z}}^{2}} .
$$

The factor $1 / R_{\mathrm{QED}}$ removes the final state corrections included in the widths $\Gamma_{\text {ee }}$ by definition (59).

\subsection{Asymmetries in fermion-pair production}

The parity violation of the neutral current results in vector and axial-vector couplings of the $\mathrm{Z}$ boson to fermions, and their measurement determines the effective weak mixing angle, $\sin ^{2} \theta_{\text {eff }}$. Since the observables widths and total cross sections do not differentiate vector and axial-vector couplings, additional observables are introduced to describe the $\cos \theta$ dependent contributions as well as the helicity dependence in equations (49) and (54). With respect to the fermion scattering angle, $\theta$, the cross sections in the forward and the backward direction, $\sigma_{\mathrm{F}}$ and $\sigma_{\mathrm{B}}$, define the forward-backward charge asymmetry, $A_{\mathrm{FB}}$,

$$
A_{\mathrm{FB}}=\frac{\sigma_{\mathrm{F}}-\sigma_{\mathrm{B}}}{\sigma_{\mathrm{F}}+\sigma_{\mathrm{B}}} .
$$

With polarized beams the helicities of the initial state fermions are given. The left-right polarization asymmetry is defined to select the part of the cross section which is odd in difference of the beam polarizations $\mathcal{P}_{\mathrm{e}^{-}}-\mathcal{P}_{\mathrm{e}^{+}}$. Introducing the effective polarization, $\mathcal{P}_{\text {eff }}=\frac{\mathcal{P}_{\mathrm{e}^{-}}-\mathcal{P}_{\mathrm{e}^{+}}}{1-\mathcal{P}_{\mathrm{e}^{+}} \mathcal{P}_{\mathrm{e}^{-}}}$, the left-right asymmetry is defined as

$$
A_{\mathrm{LR}}=\frac{\sigma\left(\mathcal{P}_{\mathrm{eff}}=-1\right)-\sigma\left(\mathcal{P}_{\mathrm{eff}}=+1\right)}{\sigma\left(\mathcal{P}_{\mathrm{eff}}=-1\right)+\sigma\left(\mathcal{P}_{\mathrm{eff}}=+1\right)} .
$$

Since at the SLC only the electron beam was polarized, the effective polarization is $\mathcal{P}_{\text {eff }}=\mathcal{P}_{\mathrm{e}^{-}}$, and $A_{\mathrm{LR}}$ takes the form

$$
A_{\mathrm{LR}}=\frac{\sigma_{\mathrm{L}}-\sigma_{\mathrm{R}}}{\sigma_{\mathrm{L}}+\sigma_{\mathrm{R}}}=A_{\mathrm{LR}}^{\text {meas }} \frac{1}{\left\langle\mathcal{P}_{\mathrm{e}}\right\rangle},
$$

where $\left\langle\mathcal{P}_{\mathrm{e}}\right\rangle$ is the average polarization of the electron beam and $A_{\mathrm{LR}}^{\text {meas }}$ is the asymmetry measured for $\mathcal{P}_{\mathrm{e}}<1$. In a similar manner, the forward-backward polarization asymmetry, $A_{\mathrm{LRFB}}$, is defined as

$A_{\mathrm{LRFB}}=\frac{\left(\sigma_{\mathrm{F}}-\sigma_{\mathrm{B}}\right)_{\mathrm{L}}-\left(\sigma_{\mathrm{F}}-\sigma_{\mathrm{B}}\right)_{\mathrm{R}}}{\left(\sigma_{\mathrm{F}}+\sigma_{\mathrm{B}}\right)_{\mathrm{L}}+\left(\sigma_{\mathrm{F}}+\sigma_{\mathrm{B}}\right)_{\mathrm{R}}}=A_{\mathrm{LRFB}}^{\mathrm{meas}} \frac{1}{\left\langle\mathcal{P}_{\mathrm{e}}\right\rangle}$,

where $A_{\mathrm{LRFB}}^{\text {meas }}$ is the forward-backward polarization asymmetry measured for $\mathcal{P}_{\mathrm{e}}<1$.

The relations between these observables and the $\mathrm{Z}$ boson couplings to fermions are expressed by introducing the asymmetry parameter $\mathcal{A}_{\mathrm{f}}$, and taking the couplings in equations (49)-(54) as real,

$$
\mathcal{A}_{\mathrm{f}}=\frac{2 g_{\mathrm{Vf}} / g_{\mathrm{Af}}}{1+\left(g_{\mathrm{Vf}} / g_{\mathrm{Af}}\right)^{2}}=\frac{2 g_{\mathrm{Vf}} g_{\mathrm{Af}}}{g_{\mathrm{Vf}}^{2}+g_{\mathrm{Af}}^{2}}=\frac{g_{\mathrm{Lf}}^{2}-g_{\mathrm{Rf}}^{2}}{g_{\mathrm{Lf}}^{2}+g_{\mathrm{Rf}}^{2}} .
$$

Deconvoluting the QED contributions and taking into account only the pure $\mathrm{Z}$ boson exchange at $\sqrt{s}=m_{\mathrm{Z}}$, the asymmetries (65)-(68) decompose into factors of coupling constants for the initial state and for the final state as follows:

$$
\begin{gathered}
A_{\mathrm{FB}}^{0, \mathrm{f}}=\frac{3}{4} \mathcal{A}_{\mathrm{e}} \mathcal{A}_{\mathrm{f}}, \\
A_{\mathrm{LR}}^{0}=\mathcal{A}_{\mathrm{e}}, \\
A_{\mathrm{LRFB}}^{0, \mathrm{f}}=\frac{3}{4} \mathcal{A}_{\mathrm{f}} .
\end{gathered}
$$

Due to the different $\mathrm{Z}$ boson couplings to right- and lefthanded fermions, colliding unpolarized electron and positron beams produces final states with a net polarization of the fermions. The polarization $\mathcal{P}_{\mathrm{f}}$ of the final state is the difference between cross sections for right- and left-handed final state helicities normalized to their sum,

$$
\mathcal{P}_{\mathrm{f}}=\frac{\mathrm{d}\left(\sigma_{\mathrm{r}}-\sigma_{\mathrm{l}}\right)}{\mathrm{d} \cos \theta} / \frac{\mathrm{d}\left(\sigma_{\mathrm{r}}+\sigma_{\mathrm{l}}\right)}{\mathrm{d} \cos \theta},
$$

yielding the average polarization of

$$
\left\langle\mathcal{P}_{\mathrm{f}}\right\rangle=\frac{\sigma_{\mathrm{r}}-\sigma_{1}}{\sigma_{\mathrm{r}}+\sigma_{\mathrm{l}}} .
$$

This allows the definition of a polarization asymmetry, $A_{\mathrm{FB}}^{\mathrm{pol}}$, for the final state fermions regarding the polarization of the final state fermions in the forward or backward hemisphere,

$$
A_{\mathrm{FB}}^{\mathrm{pol}}=\frac{\left(\sigma_{\mathrm{r}}-\sigma_{\mathrm{l}}\right)_{\mathrm{F}}-\left(\sigma_{\mathrm{r}}-\sigma_{1}\right)_{\mathrm{B}}}{\left(\sigma_{\mathrm{r}}+\sigma_{1}\right)_{\mathrm{F}}+\left(\sigma_{\mathrm{r}}+\sigma_{\mathrm{l}}\right)_{\mathrm{B}}} .
$$

The polarization of the final state fermions can only be measured for $\tau$ leptons which decay in the detector in a parity violating manner. Including only the pure $\mathrm{Z}$ boson exchange at $\sqrt{s}=m_{\mathrm{Z}}$ and deconvoluting for QED corrections, the pole asymmetries $\left\langle\mathcal{P}_{\mathrm{f}}\right\rangle$ and $A_{\mathrm{FB}}^{\mathrm{pol}}$ are related to the asymmetry 
parameters $\mathcal{A}_{\mathrm{f}}$ as

$$
\begin{gathered}
\left\langle\mathcal{P}_{\mathrm{f}}{ }^{0}\right\rangle=-\mathcal{A}_{\mathrm{f}}, \\
A_{\mathrm{FB}}^{\mathrm{pol}, 0}=-\frac{3}{4} \mathcal{A}_{\mathrm{e}} .
\end{gathered}
$$

The parameters $\mathcal{A}_{\mathrm{e}}$ and $\mathcal{A}_{\mathrm{f}}$ are specified with the variety of asymmetry measurements, and the effective vector and axialvector coupling constants, $g_{\mathrm{Vf}}$ and $g_{\mathrm{Af}}$ can be derived. The results determine the effective weak mixing angle, $\sin ^{2} \theta_{\text {eff }}$,

$$
\frac{g_{\mathrm{Vf}}}{g_{\text {Af }}}=1-\frac{2 Q_{\mathrm{f}}}{T_{3}^{\mathrm{f}}} \sin ^{2} \theta_{\mathrm{eff}}^{\mathrm{f}}=1-4\left|Q_{\mathrm{f}}\right| \sin ^{2} \theta_{\mathrm{eff}}^{\mathrm{f}} .
$$

\subsection{Pseudo-observables and model-dependence of the analyses}

The $\mathrm{Z}$ boson decay parameters are identified by analysing cross sections and asymmetries measured at the $\mathrm{Z}$ resonance. The $\mathrm{Z}$ boson mass, the $\mathrm{Z}$ boson total and partial widths are determined by the position, the height and the width of the $\mathrm{Z}$ lineshape, and the asymmetry measurements provide information on the ratio $g_{\mathrm{Vf}} / g_{\mathrm{Af}}$. As described in section 2 , the $\mathrm{Z}$ boson widths and couplings are completely defined within the model of interactions, i.e. in the Standard Model widths and couplings are specified by the parameters given in equation (46). Thus, the analysis of $\mathrm{Z}$ lineshape and asymmetries affords two results: (1) the determination of the $\mathrm{Z}$ boson decay parameters as mass, widths and couplings, in a rather model-independent way by assuming photon and $\mathrm{Z}$ boson exchange, and (2) the determination of the Standard Model parameters, in particular $m_{\mathrm{Z}}, m_{\mathrm{H}}$ and $m_{\mathrm{t}}$. For both procedures, (1) and (2), the combination of all measurements at the $Z$ resonance is required. But this is a challenge regarding the number of measurements and the different experimental conditions. It is better to perform a two-step procedure: first, cross section and asymmetry measurements are combined in a fit to derive a set of pseudo-observables that contains all relevant information on the $\mathrm{Z}$ resonance. In a second step these pseudo-observables are interpreted within the Standard Model using the result for the $\mathrm{Z}$ boson mass obtained in the first step as input parameter. The consistent description of all measurements by the extracted Standard Model parameters is a viable test of this model.

The advantage of this procedure is evident. The relations between pseudo-observables and $\mathrm{Z}$ boson parameters are simple, and the further use of pseudo-observables is easy. Since each experiment can provide its set of pseudo-observables, the combination of the measurements is straightforward. It has been verified that this two-step procedure is almost equivalent to the direct Standard Model interpretation of cross section and asymmetry measurements. This is also true for any theory beyond the Standard Model which can be absorbed in the pseudo-observables. Exceptions are theories which lead to significant modifications of the $\gamma-Z$ interference term, like models with additional neutral bosons. In these cases the first step, the extraction of $\mathrm{Z}$ pole pseudoobservables does not work because the $\gamma-Z$ interference term used for corrections depends on parameters which have to be determined in the second step.

The $Z$ pole pseudo-observables. An obvious choice for a set of pseudo-observables would be $m_{\mathrm{Z}}, \Gamma_{\mathrm{Z}}$, the partial $\mathrm{Z}$ boson decay width, $\Gamma_{\mathrm{f} \overline{\mathrm{f}}}$ and the coupling parameters, $\mathcal{A}_{\mathrm{f}}$. The observables describe the properties of the $\mathrm{Z}$ boson. However, they are substantially correlated and it is recommended to summarize the LEP measurements on the $\mathrm{Z}$ resonance using the following set of pseudo-observables:

- The mass of the $\mathrm{Z}$ boson, $m_{\mathrm{Z}}$.

- The total width of the $\mathrm{Z}$ boson, $\Gamma_{\mathrm{Z}}$.

- The hadronic pole cross section (see equation (64)),

$$
\sigma_{\text {had }}^{0}=\frac{12 \pi}{m_{\mathrm{Z}}^{2}} \frac{\Gamma_{\mathrm{ee}} \Gamma_{\mathrm{had}}}{\Gamma_{\mathrm{Z}}^{2}}
$$

that measures the inclusive hadronic final states,

$$
\sigma_{\text {had }}^{0}=\sum_{\mathrm{q} \overline{\mathrm{q}}, \mathrm{q} \neq \mathrm{t}} \sigma_{\mathrm{q} \overline{\mathrm{q}}} \text {. }
$$

- The widths parameters, $R_{\ell}^{0}$, corresponding to the ratio of hadronic and leptonic widths,

$$
R_{\ell}^{0}=\frac{\Gamma_{\text {had }}}{\Gamma_{\ell \ell}}
$$

with $\ell=\mathrm{e}, \mu, \tau . R_{\ell}^{0}$ is sensitive to the strong coupling constant $\alpha_{\mathrm{S}}$ because of the QCD correction factor $R_{\mathrm{QCD}}$ to the hadronic width (equation (60)).

- The leptonic forward-backward pole asymmetry,

$$
A_{\mathrm{FB}}^{0, \ell}=\frac{3}{4} \mathcal{A}_{\mathrm{e}} \mathcal{A}_{\ell} \text {. }
$$

with $\ell=\mathrm{e}, \mu, \tau$.

The $\tau$ polarization measurement at LEP provides the quantities $\mathcal{A}_{\mathrm{e}}$ and $\mathcal{A}_{\tau}$.

The selection of primary quarks in hadronic final states allows the determination of the ratio

$$
R_{\mathrm{q}}^{0}=\frac{\Gamma_{\mathrm{q} \overline{\mathrm{q}}}}{\Gamma_{\mathrm{had}}},
$$

in particular for $\mathrm{q}=\mathrm{b}, \mathrm{c}$. The ratio $R_{\mathrm{q}}^{0}$ corresponds to the ratio of the pole cross sections corrected for the small $\gamma$ and $\gamma-\mathrm{Z}$ interference contributions. $R_{\mathrm{b}}^{0}$ is sensitive to the top quark mass (see equation (42)), since corrections due to self-energy insertions in the propagator and QCD corrections largely cancel in the ratio $R_{\mathrm{q}}^{0}$. Assigning the charge to the primary quarks, also the peak asymmetries for $\mathrm{q} \overline{\mathrm{q}}$ final states, $A_{\mathrm{FB}}^{0, \mathrm{q}}$, can be determined and included in the analysis. The combination of measurements at SLC delivers the polarized pole asymmetries $A_{\mathrm{LR}}^{0}$ and $A_{\mathrm{LRFB}}^{0}$ corresponding to directly measured values for $\mathcal{A}_{\mathrm{e}}$ and $\mathcal{A}_{\mathrm{f}}$.

The full analysis procedure passes the following steps:

1. Combination of measurements to derive pseudoobservables:

- Combination of the lineshape and asymmetry measurements at LEP to extract the set of pseudoobservables $m_{\mathrm{Z}}, \Gamma_{\mathrm{Z}}, \sigma_{\mathrm{had}}^{0}, R_{\ell}^{0}, A_{\mathrm{FB}}^{0, \ell}$

- Determination of $\mathcal{A}_{\ell}$ from $\tau$ polarization measurements at LEP

- Combination of the measurements of the polarized asymmetries at SLC to obtain $\mathcal{A}_{\ell}$ directly

- Determination of the heavy flavour observables, $R_{\mathrm{b}}^{0}$, $R_{\mathrm{c}}^{0}, A_{\mathrm{FB}}^{0, \mathrm{~b}}, A_{\mathrm{FB}}^{0, \mathrm{c}}, \mathcal{A}_{\mathrm{b}}$ and $\mathcal{A}_{\mathrm{c}}$ from a combination of all heavy flavour measurements at LEP and SLC 


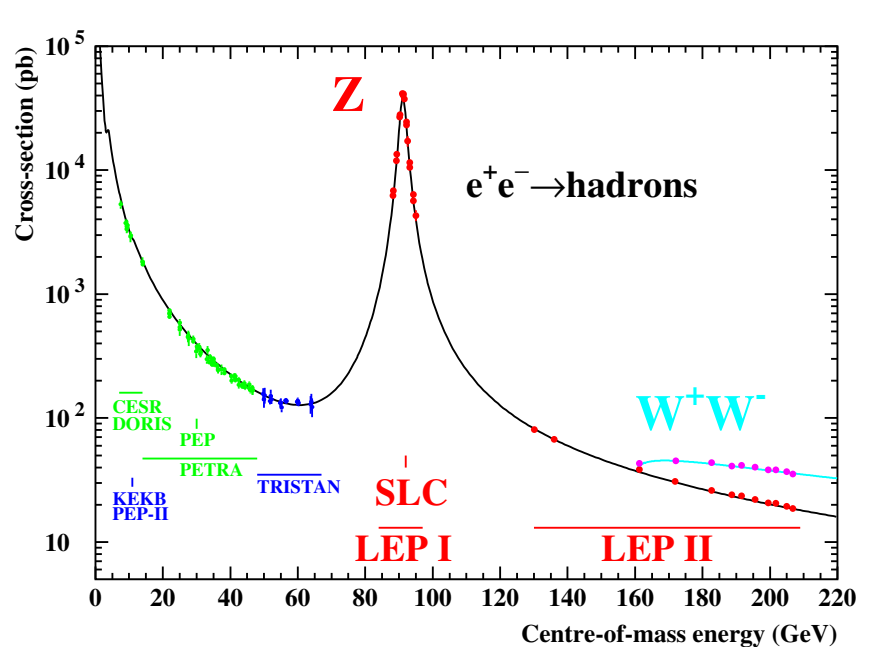

Figure 5. The hadronic cross section as a function of centre-of-mass energy [32]. The solid line is the prediction of the Standard Model and the points are the experimental measurements. The energy ranges of the various $\mathrm{e}^{+} \mathrm{e}^{-}$accelerators are also indicated. The cross sections have been corrected for the effects of photon radiation.

(Reprinted with permission from [32]. Copyright 2006 Elsevier.)

2. Determination of the $\mathrm{Z}$ boson decay parameters:

- Identify the $\mathrm{Z}$ boson partial widths

- Determine the effective $\mathrm{Z}$ boson couplings and the effective mixing angle

3. Determination of the Standard Model parameters from a fit to the pseudo-observables

4. Confirm the Standard Model by a global fit including constraints from direct measurements of the top quark and W boson mass and width

Sections 5-8 follow this chain.

\section{Measurements at $\mathrm{e}^{+} \mathrm{e}^{-}$colliders}

The Large Electron Positron (LEP) Collider [30] and the Stanford Linear Collider (SLC) [31] were designed to perform comprehensive tests of the Standard Model using electronpositron collisions at the $\mathrm{Z}$ pole. After successful running at the $\mathrm{Z}$ resonance LEP was upgraded using superconducting cavities to run at centre-of-mass energies up to $200 \mathrm{GeV}$, the so-called LEP-II phase. At these energies the weak bosons are produced pairwise. Figure 5 shows the hadronic cross section, $\mathrm{e}^{+} \mathrm{e}^{-} \rightarrow \mathrm{q} \overline{\mathrm{q}}$, as a function of the centre-of-mass energy and illustrates the energy range of LEP and SLC operation in comparison with previous $\mathrm{e}^{+} \mathrm{e}^{-}$experiments. The LEP experiments, ALEPH, DELPHI, L3 and OPAL, collected about 17 million $\mathrm{Z}$ boson events at centre-of-mass energies between 88 and $94 \mathrm{GeV}$. At the Stanford Linear Collider, SLC, the SLD experiment recorded about $5 \times 10^{5} \mathrm{Z}$ boson events in electronpositron collisions at the $\mathrm{Z}$ pole with longitudinally polarized electrons. The resulting impressive statistical uncertainties could only be exploited due to small and well understood systematic errors on luminosity, energy and polarization. The precise measurement of the absolute luminosity as well as the energy calibration were crucial for the $\mathrm{Z}$ lineshape scans at LEP. The measurement of beam polarization was decisive for the asymmetry measurements with the SLD detector at SLC. In this section, the measurements of luminosity, energy and polarization measurement are sketched.

\subsection{Precise luminosity measurement at LEP}

The cross sections are measured using the relation $\sigma=$ $N / \epsilon \mathcal{L}$, where $\mathcal{L}$ is the luminosity, $N$ the number of detected events corrected for acceptance and background and $\epsilon$ the event selection efficiency. The absolute cross section measurement requires the precise knowledge of the time integrated luminosity of the collider. In $\mathrm{e}^{+} \mathrm{e}^{-}$colliders the absolute luminosity is usually determined by comparing the $\mathrm{e}^{+} \mathrm{e}^{-} \rightarrow \mathrm{e}^{+} \mathrm{e}^{-}$(Bhabha) cross section measured at small angles with its theoretical prediction. At small polar angles the Bhabha cross section is given by the photon exchange in the $t$-channel,

$$
\frac{\mathrm{d} \sigma}{\mathrm{d} \theta} \approx \frac{32 \pi \alpha^{2}}{s} \frac{1}{\theta^{3}},
$$

and can be calculated to the precision of $\mathcal{O}\left(10^{-4}\right)$ [33]. The experimental challenge is the definition of the geometrical acceptance. The measurement of the Bhabha cross section integrated over an interval at small angles $(\theta \approx 0)$ results in

$$
\sigma_{\text {Bhabha }} \approx \frac{16 \pi \alpha^{2}}{s}\left(\frac{1}{\theta_{\min }^{2}}-\frac{1}{\theta_{\max }^{2}}\right)
$$

and is very sensitive to the minimum acceptance angle $\theta_{\min }$. Thus, the luminosity measurement is transferred into a position measurement of the Bhabha signal in the very-forward region of the detector. Knowing both, the absolute and relative positions of the luminosity detectors, at the 10-20 $\mu \mathrm{m}$ level the LEP experiments have achieved an experimental error, $(\delta \mathcal{L} / \mathcal{L})_{\text {exp }}$, well below $0.1 \%$. The theory error has improved during the years of data taking from $0.24 \%$ to $0.07 \%$ to match this impressive experimental uncertainty. For details of the luminosity measurement using Bhabha scattering see the analyses given in [34-38].

\subsection{Energy measurement at LEP}

The precision reached at LEP was strongly enhanced by the precise measurement of the beam energy based on the technique of resonant depolarization. Transverse polarization builds up in storage rings by the Sokolov-Ternov effect [39]. Depolarization effects are caused by synchrotron radiation and unavoidable imperfections in the storage ring. In an ideal $\mathrm{e}^{+} \mathrm{e}^{-}$machine with purely vertical magnetic fields the vertical component of the spin vectors is conserved and a large asymptotic transverse polarization builds up slowly. The spins of the particles precess around the vertical direction with a frequency $f_{\text {spin }}$,

$$
f_{\text {spin }}=v \cdot f_{\text {rev }}
$$

where $f_{\text {rev }}$ is the revolution frequency and the spin tune $v$ is given by

$$
v=\frac{g-2}{2} \gamma=\frac{E_{\text {beam }}[\mathrm{MeV}]}{440.6486 \mathrm{MeV}}
$$


By applying an oscillating horizontal weak dipole field, the spins are slightly rotated away from the vertical direction on each turn. A depolarizing resonance occurs if the the depolarizing RF field is in phase with the spin precession,

$$
f_{\text {depol }}=(k \pm[\nu]) f_{\text {rev }},
$$

where $k$ is an integer and [v] the non-integer part of the spin tune. The depolarizing field is applied once per turn, therefore the resonance appears at a frequency which is independent of the integer part of the spin tune. The average beam energy is proportional to the average spin tune,

$$
E_{\text {beam }}(\mathrm{MeV})=440.6486 \mathrm{MeV}\left(N_{\mathrm{S}}+\frac{f_{\text {depol }}}{f_{\text {rev }}}\right) .
$$

The numerical coefficient in equation (89) is known to the relative precision of $2 \times 10^{-7}$. The integer part of the spin tune, $N_{\mathrm{S}}$, depends on the experimental facility. At LEP it was deduced from the calibration of the bending magnet resulting in $N_{\mathrm{S}}=103$ at the $\mathrm{Z}$ pole. Details on the energy measurement at LEP-I can be found in [40], the spin dynamics at LEP is summarized in [41].

With the method of resonant depolarization an energy uncertainty at the $\mathrm{Z}$ pole of $\delta \mathrm{E}_{\mathrm{cm}} \leqslant 0.8 \mathrm{MeV}$ was achieved corresponding to a relative accuracy of $\approx 1 \times 10^{-5}$. This impressive result allowed the identification of systematic effects as earth tides and electrical currents from the high speed train TGV passing by near the ring. Since resonant depolarization gives an accurate value only at the time of measurement, energy fluctuations caused by temperature effects, orbit corrections and other stress effects had to be tracked and included in a model of corrections.

In a real beam, the energy of individual particles oscillates around the mean energy. Therefore, the measurements are averaged over the corresponding centre-of-mass energy range, $E_{\mathrm{cm}}=E_{\mathrm{cm}}^{0} \pm \delta E_{\mathrm{cm}}^{\mathrm{spread}}$. Assuming a Gaussian shape of the energy distribution, the cross sections, $\sigma\left(E_{\mathrm{cm}}^{2}\right)$, receive a correction. At LEP-I typical values of the centre-of-mass energy spread were giving additional rise to the uncertainty of the precision observables.

From 1995 to 2000, in the LEP-II phase, the energy was increased successively from 130 to $208 \mathrm{GeV}$. However, the transverse polarization did not build up at LEP for beam energies above $60 \mathrm{GeV}$. The problem was the energy spread which rises quadratically with the beam energy, $E_{\text {beam }}^{2}$, and reached almost the same magnitude as the $440 \mathrm{MeV}$ energy spacing between two consecutive linear spin resonances. With higher LEP-II energies particles in the tails of the beam energy distribution experienced strong depolarizing forces and the required polarization could not build up. The energy measurement was performed using 16 NMR (nuclear magnetic resonance) probes installed in the LEP dipoles for continuous measurement of the bending magnetic field. The relation between the fields measured by the probes and the beam energy was defined in a NMR model, which was calibrated against precise measurements of the average beam energy between 41 and $60 \mathrm{GeV}$ made using the resonant depolarization technique. With this procedure an accuracy of $20 \mathrm{MeV}$ was reached at beam energies of $90 \mathrm{GeV}$.

\subsection{Polarization measurement at $S L C$}

In contrast to LEP, the SLC provided collisions of polarized electrons with unpolarized positrons at the $\mathrm{Z}$ pole. The longitudinally polarized electrons were generated by photoemission from a GaAs cathode using circularly polarized light produced in a Ti-sapphire laser. A high degree of polarization of almost $80 \%$ was achieved using a 'strained lattice' GaAs photocathode, where the mechanical strain removes a degeneracy in the valence band. The helicity of the electrons was chosen randomly pulse-to-pulse by controlling the circular polarization of the laser. In the years 1993-1998 the average polarization of the electron beam reached $\approx 75 \%$. The measurement of left-right asymmetries is less demanding concerning the knowledge of the absolute luminosity. However, the precise determination of the average luminosity-weighted polarization, $\left\langle\mathcal{P}_{\mathrm{e}}^{\text {lum }}\right\rangle$, is essential,

$$
A_{\mathrm{LR}}=\frac{\sigma_{\mathrm{L}}-\sigma_{\mathrm{R}}}{\sigma_{\mathrm{L}}+\sigma_{\mathrm{R}}}=\frac{N_{\mathrm{L}}-N_{\mathrm{R}}}{N_{\mathrm{L}}+N_{\mathrm{R}}} \frac{1}{\left\langle\mathcal{P}_{\mathrm{e}}^{\operatorname{lum}}\right\rangle},
$$

where $N_{\mathrm{L}, \mathrm{R}}$ are the numbers of detected events for left- or righthanded electron beam polarization, corrected for acceptance and background. The polarization was measured using a Compton polarimeter exploiting the Compton process e $\gamma \rightarrow$ $\mathrm{e} \gamma$, the cross section of which depends on the polarization of electrons and photons. The polarimeter could be operated continually while beams were in collision. Downstream of the $\mathrm{e}^{+} \mathrm{e}^{-}$interaction point the circularly polarized laser beam was brought into collision with the outgoing electron beam to measure the polarization. However, the polarization measured at the Compton interaction point was different from that at the $\mathrm{e}^{+} \mathrm{e}^{-}$collision point mainly for the following reasons: (1) the small beam sizes at the interaction point induce beam-beam interactions that change the polarization before it could be measured in the polarimeter. (2) Due to the energy dispersion of the electrons in the bunch, the spin precession in the arcs at the SLC was different for off-energy electrons resulting in reduced longitudinal polarization. These off-energy electrons did not focus to the small interaction point and contributed less to the luminosity, but they contributed as much as onenergy electrons to the beam polarization measurement. Both effects were evaluated in dedicated beam transport studies and taken into account to determine the averaged luminosityweighted polarization. An impressive overall uncertainty on the luminosity-weighted polarization amounting to $0.5-0.6 \%$ for the years 1994-1998 was achieved, the details can be found in $[42,43]$. The zero polarization of the positron beam was confirmed exploring the polarization dependent asymmetries in Bhabha scattering using a Møller(Bhabha) polarimeter [44].

\subsection{Measurements at future $e^{+} e^{-}$colliders}

The LHC results will allow a first look at Terascale physics. Whatever the outcome will be, in all cases precision measurements at high energies will be essential to a full understanding of the phenomena. Precision experiments at high-energy $\mathrm{e}^{+} \mathrm{e}^{-}$colliders will distinguish among patterns of new physics scenarios providing a telescopic view of physics even beyond the LHC energy reach. 
Future $\mathrm{e}^{+} \mathrm{e}^{-}$accelerators beyond LEP-II energies must be linear to avoid the enormous energy loss by synchrotron radiation. The high luminosity at high energies can only be achieved if intense beams of extremely small cross sections collide. Projects for future linear colliders are the International Linear Collider (ILC) [45] to begin operation at 200-500 GeV with the potential to upgrade to $1 \mathrm{TeV}$ thereafter, and the Compact Linear Collider (CLIC) $[46,47]$ for multi-TeV energies. It is desired that also a high-luminosity run at the $\mathrm{Z}$ pole will be possible (see section 7.10).

The precision measurements planned at these colliders will require huge efforts to precisely measure the energy, luminosity and polarization. The luminosity will be measured using Bhabha scattering, and the beam polarization will be determined using Compton polarimetry. A precision at the per-mille level is expected for these measurements [45], comparable or even better than the precision reached at LEP and SLC. But it will be impossible to apply the method of resonant depolarization to measure the beam energy. Current studies [45] show that the precision for the energy measurement at future linear colliders will be at the level $10^{-4}$, substantially below the precision reached at LEP-I.

The measurements at linear future colliders will highly benefit from polarization of both beams, electrons and positrons [48]. A well-known initial state provides additional sensitivity to difficult signatures and increases the precision due to additional constraints. The corresponding design to produce polarized beams and to measure the polarization with excellent accuracy are currently under study (see references [45, 49] and references therein).

\section{Precision measurements at the $\mathrm{Z}$ resonance}

The LEP experiments, ALEPH, DELPHI, L3 and OPAL, collected in total 15.5 million $\mathrm{Z}$ decays to inclusive hadronic final states and 1.7 million $\mathrm{Z}$ decays to the leptonic final states at centre-of-mass energies between 88 and $94 \mathrm{GeV}$. These data have been analysed to measure the $\mathrm{Z}$ lineshape and the leptonic forward-backward charge asymmetries. Special treatment and procedures allowed the selection of exclusive $q \bar{q}$ final states and to measure the widths ratio $R_{\mathrm{q}}$ as well as the forwardbackward charge asymmetries for quark final states.

Complementarily, 0.55 million $\mathrm{Z}$ events were collected in collisions of polarized electrons with unpolarized positrons with the SLD detector at SLC affording the measurements of left-right asymmetries as well as forward-backward polarization asymmetries for leptonic and exclusive $q \bar{q}$ final states.

\subsection{Measurement of the $Z$ lineshape}

Measurements of the total cross sections were performed for the leptonic final states, $\mathrm{e}^{+} \mathrm{e}^{-}, \mu^{+} \mu^{-}$and $\tau^{+} \tau^{-}$, and for the inclusive hadronic final state arising from $q \bar{q}$ production. The final states were separated based on their different topologies; hadronic events are characterized by high-multiplicity jets arising from quark fragmentation, and they deposit large visible energy in the detector whereas leptonic events feature low-multiplicity tracks in the detector. Electrons and muons are selected as two high-energy tracks oriented back-toback; electrons deposit their energy in the electromagnetic calorimeter, muons traverse all subdetectors as minimum ionizing particles, and their momenta were measured using the inner and outer tracking systems. Tau leptons decay near the interaction point and are identified by low-multiplicity signatures different from di-electron, di-muon and from the high-multiplicity signature of quark jets in hadronic events and taking into account the missing energy due to neutrinos in the $\tau$ lepton decay chain.

Due to the advanced detectors with almost $4 \pi$ coverage, backgrounds in the selected data samples were typically at the $1 \%$ level, and the event selection efficiencies ranged from $70 \%$ for $\tau^{+} \tau^{-}$final states up to more than $99 \%$ for the inclusive hadronic final state. The large size of the data sample and the high event selection efficiency imply very small statistical uncertainties. The cross section uncertainties are at the level of $0.05 \%$ for the inclusive hadronic channel and about $0.25 \%$ for the lepton channels. The systematic uncertainties, mainly due to event selection, luminosity and energy measurement as well as theoretical uncertainties, need to match this precision. The systematic errors due to event selection efficiency given by each of the LEP experiments are typically $0.04 \%$ to $0.1 \%$ for the inclusive hadronic final states, and are slightly larger for the leptonic final states up to $0.7 \%$ especially for tau pairs. The uncertainty of the luminosity measurement (see section 4.1) is below the statistical errors. The uncertainties from the energy measurement, $\delta E_{\mathrm{cm}} \leqslant 0.8 \mathrm{MeV}$ and the beam energy spread which was at LEP-I around $50 \mathrm{MeV}$ have been included in the analyses. The effect of initial state radiation of photons reduces the effective centre-of-mass energy and is described at the per-mille level and better by the precision programs ZFITTER [23] and TOPAZ0 [24]. Deconvoluting the QED radiative corrections the improved Born cross section has been deduced, and correcting for the contributions from photon exchange and the $\gamma-Z$ interference, the pure $Z$ resonance has been separated. The correspondence between measurements, pure $\mathrm{Z}$ boson resonance and pseudo-observables is depicted in the left plot of figure 6 for the inclusive hadronic cross section. This figure demonstrates impressively the influence of initial state radiation.

\subsection{Measurement of forward-backward charge asymmetries}

The measurement of the differential cross section requires the charge discrimination of the final state fermions and anti-fermions. For the $\mu^{+} \mu^{-}$final state this can be done directly, $\mathrm{e}^{+} \mathrm{e}^{-}$final states have to be corrected for the $t$-channel contributions. The charge of the final state $\tau$ leptons is derived from their decay products.

The differential cross section for unpolarized beams can be described using the forward-backward charge asymmetry,

$$
\frac{\mathrm{d} \sigma}{\mathrm{d} \cos \theta} \propto 1+\cos ^{2} \theta+\frac{8}{3} A_{\mathrm{FB}}^{\mathrm{meas}}(s) \cos \theta,
$$

where $A_{\mathrm{FB}}^{\text {meas }}(s)$ is the measured forward-backward asymmetry that includes initial state radiation. The LEP experiments 

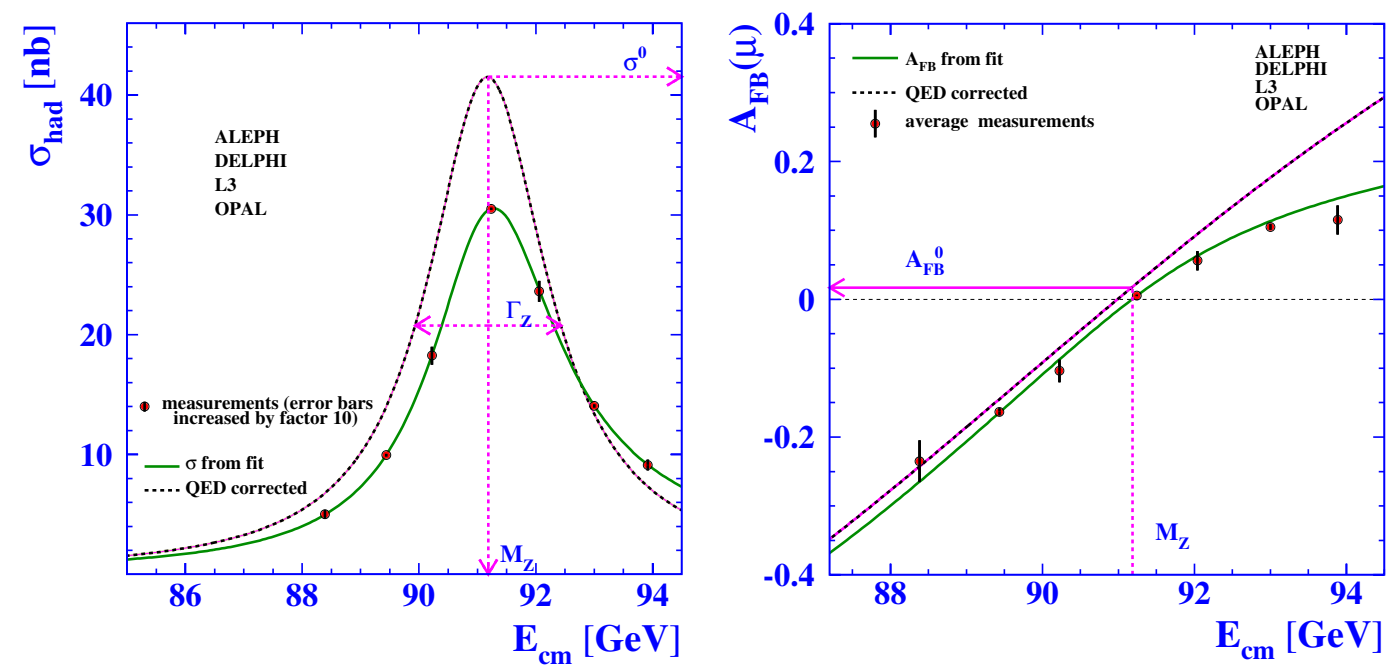

Figure 6. Left: average over measurements of the total inclusive hadronic cross section of the process $\mathrm{e}^{+} \mathrm{e}^{-} \rightarrow \mathrm{q} \overline{\mathrm{q}}$. Right: average over measurements of the forward-backward asymmetry $A_{\mathrm{FB}}^{\mu}$ in muon production, $\mathrm{e}^{+} \mathrm{e}^{-} \rightarrow \mu^{+} \mu^{-}$. The full lines represent the result of a fit including QED radiative corrections to the measurements. Unfolding for QED radiative effects yields the dashed curves, corresponding to the parameters described in section 3.5. See also [32]. (Reprinted with permission from [32]. Copyright 2006 Elsevier.)

measured the leptonic forward-backward asymmetries for $\mathrm{e}^{+} \mathrm{e}^{-}, \mu^{+} \mu^{-}, \tau^{+} \tau^{-}$final states. In the case of the Bhabha process, the contributions from boson exchange in the $t$ channel have been subtracted to extract the pole asymmetry $A_{\mathrm{FB}}^{0, \mathrm{e}}$.

The experimental systematic errors are between two and five times smaller than the statistical uncertainties. The improved Born forward-backward asymmetries have been derived by deconvoluting the QED radiative corrections. The pole asymmetry is given for $\sqrt{s}=m_{\mathrm{Z}}$ and correcting for small contributions from photon exchange. This procedure is demonstrated in the right plot of figure 6 for the asymmetry measurements of $\mu^{+} \mu^{-}$final states performed at LEP. The results of the measurements are shown as a function of $\sqrt{s}$ as well as the asymmetries after unfolding for QED radiative effects. The corresponding $\mathrm{Z}$ pole parameters are described in section 3.5 .

\subsection{The parameters of the $Z$ lineshape}

It would be an ideal solution to combine all cross sections and leptonic forward-backward asymmetries measured by each of the four LEP experiments. But their correlations are very complex and it is difficult to support a clear combination procedure. A more successful approach is the combination of the pseudo-observables, $m_{\mathrm{Z}}, \Gamma_{\mathrm{Z}}, \sigma_{\mathrm{had}}^{0}, R_{\ell}^{0}$ and $A_{\mathrm{FB}}^{0, \ell}$ $(\ell=\mathrm{e}, \mu, \tau)$, after they have been provided by each of the experiments. By assuming universality of the charged leptons in neutral current reactions these nine pseudo-observables reduce to five. The combined LEP results for the pseudoobservables are summarized in table 1 . They comprise the information on the $\mathrm{Z}$ boson measured at the $\mathrm{Z}$ boson resonance.

\subsection{Tau polarization at LEP}

As introduced in section 3.4, $\mathrm{e}^{+} \mathrm{e}^{-}$interactions with unpolarized beams yield a net final state fermion polarization $\mathcal{P}_{\mathrm{f}}$ which can be described in terms of the coupling
Table 1. Combined results from LEP measurements at the $\mathrm{Z}$ resonance, with and without the assumption of lepton universality [32].

\begin{tabular}{lcc}
\hline Parameter & Without lepton universality & With lepton universality \\
\hline$m_{\mathrm{Z}}(\mathrm{GeV})$ & $91.1876 \pm 0.0021$ & $91.1875 \pm 0.0021$ \\
$\Gamma_{\mathrm{Z}}(\mathrm{GeV})$ & $2.4952 \pm 0.0023$ & $2.4952 \pm 0.0023$ \\
$\sigma_{\mathrm{had}}^{0}(\mathrm{nb})$ & $41.541 \pm 0.037$ & $41.540 \pm 0.037$ \\
$R_{\mathrm{e}}^{0}$ & $20.804 \pm 0.050$ & \\
$R_{\mu}^{0}$ & $20.785 \pm 0.033$ & \\
$R_{\tau}^{0}$ & $20.764 \pm 0.045$ & \\
$R_{\ell}^{0}$ & & \\
$A_{\mathrm{FB}}^{0, \mathrm{e}}$ & $0.0145 \pm 0.0025$ & \\
$A_{\mathrm{FB}}^{0, \mu}$ & $0.0169 \pm 0.0013$ & \\
$A_{\mathrm{FB}}^{0, \tau}$ & $0.0188 \pm 0.0017$ & \\
$A_{\mathrm{FB}}^{0, \ell}$ & & $0.0171 \pm 0.0010$ \\
\hline
\end{tabular}

parameters, $\mathcal{A}_{\mathrm{f}}$. Taking into account only the contribution from the $\mathrm{Z}$ boson exchange, one finds for the angular distribution of $\mathcal{P}_{\mathrm{f}}$ for $\sqrt{s}=m_{\mathrm{Z}}$,

$$
\mathcal{P}_{\mathrm{f}}(\cos \theta)=-\frac{\mathcal{A}_{\mathrm{f}}\left(1+\cos ^{2} \theta\right)+2 \mathcal{A}_{\mathrm{e}} \cos \theta}{\left(1+\cos ^{2} \theta\right)+2 \mathcal{A}_{\mathrm{f}} \mathcal{A}_{\mathrm{e}} \cos \theta} .
$$

When averaged over all scattering angles $\theta$ the pole polarization $\left\langle\mathcal{P}_{\mathrm{f}}\right\rangle=-\mathcal{A}_{\mathrm{f}}$ is measured (see equation (76)), averaging over the forward and backward hemispheres allows the measurement of $A_{\mathrm{FB}}^{\mathrm{pol}, 0}$ (equation (77)). Only the process $\mathrm{e}^{+} \mathrm{e}^{-} \rightarrow \tau^{+} \tau^{-}$affords access to the helicity of the final state lepton. The parity-violating coupling of the exchanged $\mathrm{Z}$ boson to initial state electrons and final state $\tau$ leptons affects the distribution of the decay angles in the rest frame of the $\tau$ lepton. This translates into an energy spectrum of the decay products in the laboratory (detector) system that depends on the average helicity of the $\tau$ lepton.

All LEP experiments have analysed the most important $\tau$ decay modes with high branching fractions [50-53]: $\tau \rightarrow \pi \nu$, 


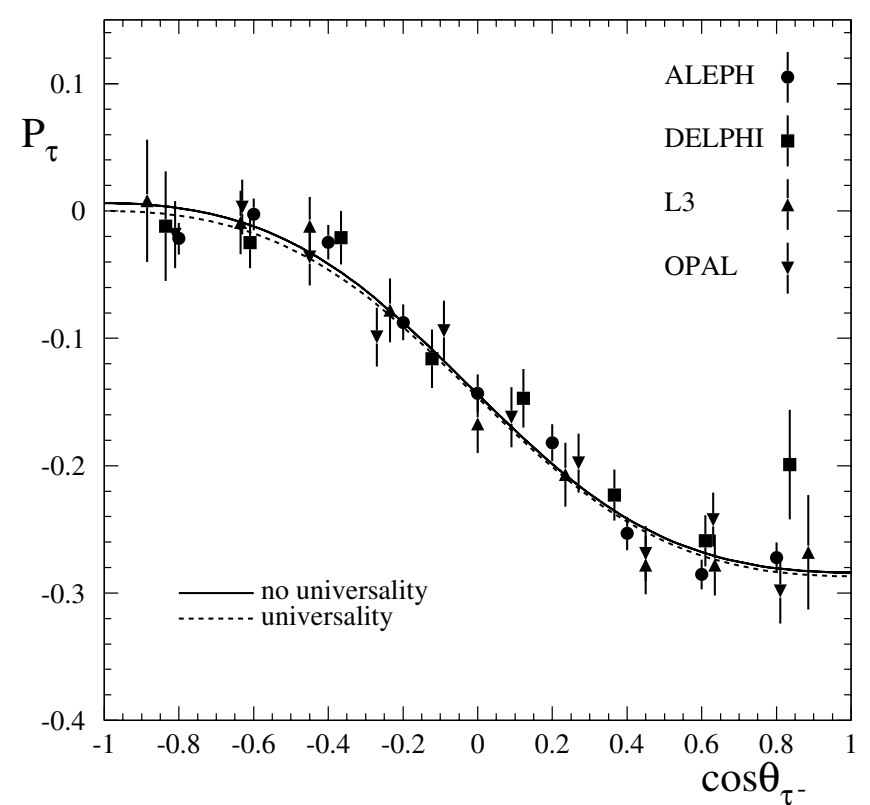

Figure 7. The $\tau$ polarization as a function of $\cos \theta_{\tau^{-}}$as measured by each of the LEP experiments [32]. Only the statistical errors are shown. The solid curve overlays equation (92) for the LEP values of $\mathcal{A}_{\tau}$ and $\mathcal{A}_{\mathrm{e}}$, and the dashed curve for the LEP value of $\mathcal{A}_{\ell}$ under the assumption of lepton universality. (Reprinted with permission from [32]. Copyright 2006 Elsevier.)

$\tau \rightarrow \rho \nu, \tau \rightarrow \mathrm{a}_{1} \nu, \tau \rightarrow \mathrm{e} \nu \bar{\nu}$ and $\tau \rightarrow \mu \nu \bar{\nu}$, and extracted the $\tau$ polarization. Each of the most sensitive channels, $\rho \nu$ and $\pi v$, contributes with a weight of about $40 \%$ to the average of the measured $\tau$ polarization.

The measurement of $\mathcal{P}_{\mathrm{f}}(\cos \theta)$ allows the determination of both, $\mathcal{A}_{\mathrm{e}}$ and $\mathcal{A}_{\tau}$, providing a test of universality of the $\mathrm{Z}$ boson couplings to electrons and $\tau$ leptons. Figure 7 shows the $\tau$ polarization measured as a function of $\cos \theta_{\tau^{-}}$by the individual experiments. Based on equation (92) values of $\mathcal{A}_{\tau}$ and $\mathcal{A}_{\mathrm{e}}$ have been derived by each experiment and then combined $[32,50-53]$. Taking into account the systematic uncertainties from QED radiative corrections and extrapolations to the $\mathrm{Z}$ pole, and branching fractions from hadronic $\tau$ decay models, the combined LEP results for $\mathcal{A}_{\mathrm{e}}$ and $\mathcal{A}_{\tau}$ are

$$
\begin{aligned}
& \mathcal{A}_{\tau}=0.1439 \pm 0.0043, \\
& \mathcal{A}_{\mathrm{e}}=0.1498 \pm 0.0049 .
\end{aligned}
$$

The errors of $\mathcal{A}_{\mathrm{e}}$ and $\mathcal{A}_{\tau}$ are dominated by statistical uncertainties. The larger error of $\mathcal{A}_{\mathrm{e}}$ is related to the measurement of the forward-backward asymmetry of the $\tau$ polarization; it requires the charge discrimination between the final state $\tau^{+}$and $\tau^{-}$resulting in a lower selection efficiency. The values for $\mathcal{A}_{\mathrm{e}}$ and $\mathcal{A}_{\tau}$ are compatible with lepton universality and can be combined to give the leptonic asymmetry parameter

$$
\mathcal{A}_{\ell}=0.1465 \pm 0.0032 .
$$

The corresponding graph for the $\tau$ polarization, $\mathcal{P}_{\tau}\left(\cos \theta_{\tau^{-}}\right)$, is overlaid in figure 7 using the combined LEP values of $\mathcal{A}_{\tau}$ and $\mathcal{A}_{\mathrm{e}}$ as well as $\mathcal{A}_{\ell}$ f or the assumption of lepton universality.

\subsection{Polarized asymmetries at SLC}

The SLC provided longitudinally polarized electrons for $\mathrm{e}^{+} \mathrm{e}^{-}$ collisions at the $\mathrm{Z}$ pole, the centre-of-mass energy with the highest annihilation cross section. Routinely a polarization between $73 \%$ and $77 \%$ was achieved. The positron beam was unpolarized.

Left-right asymmetry. The measurement of the left-right asymmetry at the $\mathrm{Z}$ pole allows the direct determination of the coupling parameter for electrons. It is an important advantage that each of the fermion-pair final states can be used to determine $A_{\mathrm{LR}}^{0}=\mathcal{A}_{\mathrm{e}}$, except the Bhabha process with nonresonant $t$-channel contributions. Thus, the SLD collaboration performed the $A_{\mathrm{LR}}$ measurement using the high-statistics inclusive hadronic channel, $\mathrm{e}^{+} \mathrm{e}^{-} \rightarrow \mathrm{q} \overline{\mathrm{q}}$ [54]. The left-right asymmetries measured at SLC have been converted into $A_{\mathrm{LR}}^{0}=$ $\mathcal{A}_{\mathrm{e}}$ by including the luminosity-weighted polarization, $\left\langle\mathcal{P}_{\mathrm{e}}\right\rangle$ (see section 4.3),

$$
A_{\mathrm{LR}}=\frac{N_{\mathrm{L}}-N_{\mathrm{R}}}{N_{\mathrm{L}}+N_{\mathrm{R}}} \frac{1}{\left\langle\mathcal{P}_{\mathrm{e}}\right\rangle},
$$

and correcting for the contributions from initial state radiation and the $\gamma-\mathrm{Z}$ interference terms,

$$
A_{\mathrm{LR}}^{0} \equiv A_{\mathrm{LR}}+\Delta A_{\mathrm{LR}}
$$

Expression (94) applies only for the ideal case without systematic errors but it is a good approximation. $A_{\mathrm{LR}}$ is a robust quantity, since most systematic effects, such as those affecting efficiency or acceptance, cancel out. The largest systematic uncertainty arises from the polarization measurement which has been performed with an accuracy of $\leqslant 0.65 \%$ using a Compton polarimeter (see section 4.3). All sources of systematic errors have been evaluated and taken into account, the resulting systematic error of $A_{\mathrm{LR}}$ is below the statistical uncertainty. The complete SLD data sample yields

$$
A_{\mathrm{LR}}^{0}=0.1514 \pm 0.0022 \text {. }
$$

More details can be found in [32, 54].

Leptonic left-right forward-backward asymmetry. The leftright forward-backward asymmetries, $A_{\mathrm{LRFB}}^{\mathrm{f}}$, have been measured for specific final states $\mathrm{f} \overline{\mathrm{f}}$ at SLC. After the correction for photonic contributions, and taking into account the electron polarization, the asymmetry parameters $\mathcal{A}_{\mathrm{f}}$ have been determined from the relation

$$
A_{\mathrm{LRFB}}^{0, \mathrm{f}}=\frac{3}{4} \mathcal{A}_{\mathrm{f}}
$$

The measurements of the final states $\mathrm{e}^{+} \mathrm{e}^{-}, \mu^{+} \mu^{-}$and $\tau^{+} \tau^{-}$at SLC have been used to determine the asymmetry parameters $\mathcal{A}_{\ell}$ for the three charged lepton species; the event selection and charge discrimination and the analysis are described in $[55,56]$. In the case of Bhabha scattering, the additional contributions due to boson exchange in $t$-channel scattering have been taken into account. 
Leptonic asymmetry parameters from SLC. The combined results of the $A_{\mathrm{LR}}^{0}$ and $A_{\mathrm{LRFB}}^{0, \ell}$ measurements are

$$
\begin{gathered}
\mathcal{A}_{\mathrm{e}}=0.1516 \pm 0.0021, \\
\mathcal{A}_{\mu}=0.142 \pm 0.015, \\
\mathcal{A}_{\tau}=0.136 \pm 0.015 .
\end{gathered}
$$

The agreement of these values supports lepton universality, resulting in

$$
\mathcal{A}_{\ell}=0.1513 \pm 0.0021 \text {. }
$$

The impressive accuracy for $\mathcal{A}_{\mathrm{e}}$ is due to the precise $A_{\mathrm{LR}}^{0}$ measurement which dominates the analysis.

\subsection{Heavy quark flavours at LEP and SLC}

The $\mathrm{Z}$ lineshape analyses in the inclusive hadronic channel $\mathrm{e}^{+} \mathrm{e}^{-} \rightarrow$ hadrons do not allow the examination of particular $\mathrm{q} \overline{\mathrm{q}}$ final states separately. To extract the $\mathrm{Z}$ boson couplings to individual quark flavours these specific final hadronic states have to be selected by sophisticated tagging methods. This is possible because of three main features: (1) b- and c-quarks decay weakly, therefore the hadrons have non-zero lifetimes and their decay vertex can be separated from the primary vertex using a precise vertex detector, (2) b- and c-quarks decay semileptonically, electron and muon can be identified, (3) c hadrons can be reconstructed via their decay products.

For the heavy quark flavours, similar pseudo-observables as for leptonic final states can be measured at the $\mathrm{Z}$ pole,

$$
\begin{gathered}
R_{\mathrm{q}}^{0}=\frac{\Gamma_{\mathrm{q} \overline{\mathrm{q}}}}{\Gamma_{\mathrm{had}}}, \\
A_{\mathrm{FB}}^{0, \mathrm{q}}=\frac{3}{4} \mathcal{A}_{\mathrm{e}} \mathcal{A}_{\mathrm{q}}, \\
A_{\mathrm{LRFB}}^{0, \mathrm{q}}=\frac{3}{4} \mathcal{A}_{\mathrm{q}} .
\end{gathered}
$$

For the $\mathrm{b} \overline{\mathrm{b}}$ and $\mathrm{c} \overline{\mathrm{c}}$ final states, $R_{\mathrm{q}}^{0}$ and $A_{\mathrm{FB}}^{0, \mathrm{q}}$ have been measured at LEP and $A_{\mathrm{LRFB}}^{0, \mathrm{q}}$ at SLD. In addition, the collaborations DELPHI [57] and SLD [58] measured $A_{\mathrm{FB}}^{0, \mathrm{q}}$ and $\mathcal{A}_{\mathrm{q}}$ for $\mathrm{q}=\mathrm{s}$; and OPAL [59] determined $A_{\mathrm{FB}}^{0, \mathrm{q}}$ and the branching fractions for $\mathrm{Z}$ boson decays into light quarks, for $\mathrm{q}=\mathrm{u}, \mathrm{d}$, $\mathrm{s}$. However, these measurements are much less precise than those for $q=b, c$. A detailed description of the selection and combination procedures including the treatment of all uncertainties can be found in [32] and in the report [60]. Here, only the principle of heavy flavour analysis is sketched.

Measurements of partial width. The measurement of the heavy quark partial widths, $R_{\mathrm{b}}^{0}=\Gamma_{\mathrm{b} \overline{\mathrm{b}}} / \Gamma_{\text {had }}$ and $R_{\mathrm{c}}^{0}=$ $\Gamma_{\mathrm{c} \overline{\mathrm{c}}} / \Gamma_{\text {had }}$, requires the identification of the underlying quark flavour in hadronic events. Among the $q \bar{q}$ final states, those containing heavy quarks can be identified with a certain efficiency tuned to be high for b- and c-quarks but low for uds-quarks. The heavy quark final states can be tagged by the relatively large invariant masses of the decay products, by their semileptonic decays or by their long lifetimes leading to secondary vertices. The latter can either be reconstructed directly or tagged by high impact parameters measuring whether a track originates from the decay of a long-lived particle. At SLC the small beam pipe enabled excellent b-tagging performance, i.e. high efficiency with high purity of the selected data sample. The heavy quarks can be identified in only one or in both hemispheres, so the fraction $f_{\mathrm{s}}$ or $f_{\mathrm{d}}$ of single- or double-tagged $\mathrm{Z} \rightarrow \mathrm{b} \overline{\mathrm{b}}$ events can be expressed by

$$
\begin{gathered}
f_{\mathrm{s}}=\varepsilon_{\mathrm{b}} R_{\mathrm{b}}+\varepsilon_{\mathrm{c}} R_{\mathrm{c}}+\varepsilon_{\mathrm{uds}}\left(1-R_{\mathrm{b}}-R_{\mathrm{c}}\right), \\
f_{\mathrm{d}}=\varepsilon_{\mathrm{b}}^{(d)} R_{\mathrm{b}}+\varepsilon_{\mathrm{c}}^{(d)} R_{\mathrm{c}}+\varepsilon_{\mathrm{uds}}^{(d)}\left(1-R_{\mathrm{b}}-R_{\mathrm{c}}\right),
\end{gathered}
$$

with the tagging efficiency $\varepsilon_{\mathrm{f}}$ for quark flavour $\mathrm{f}$. The doubletagging efficiency $\varepsilon_{\mathrm{f}}^{(\mathrm{d})}$ is

$$
\varepsilon_{\mathrm{f}}^{(\mathrm{d})}=\left(1+\mathcal{C}_{\mathrm{f}}\right) \varepsilon_{\mathrm{f}}^{2}
$$

taking into account that the tagging efficiencies between the hemispheres may be correlated, $\mathcal{C}_{\mathrm{f}} \neq 0$. Without correlation and neglecting all backgrounds, the width parameter $R_{\mathrm{b}}$ becomes a simple expression,

$$
R_{\mathrm{b}}=f_{\mathrm{s}}^{2} / f_{\mathrm{d}} \approx N_{\mathrm{t}}^{2} /\left(4 N_{\mathrm{tt}} N_{\mathrm{had}}\right)
$$

with the numbers $N_{\mathrm{t}}$ and $N_{\mathrm{tt}}$ of single and double tagged hadronic events with b-quarks. Hence $R_{\mathrm{b}}$ is independent of the tagging efficiencies and can be determined directly from data. However, the neglected terms are not small. The b-quark sample contains background from c-quarks. Corrections dependent on the background tagging efficiencies $\varepsilon_{\mathrm{c}}, \varepsilon_{\text {uds }}$ and hemisphere correlations must be applied, they have to be determined by Monte Carlo simulations and calibrated with data. In multi-tag methods, different tagging algorithms are used simultaneously to improve the b-tagging performance. The high purity increases the precision and reduces the sensitivity to the background efficiencies and hemisphere correlations.

The tagging of c-quarks is more complicated, and especially at LEP due to the larger beam pipe, less efficient and less pure than for b-quarks. Due to the cascade decay, $\mathrm{b} \rightarrow \mathrm{c} \rightarrow \mathrm{X}$, the reconstruction of $\mathrm{D}$ mesons enriches also the b-quark content and the results for $R_{\mathrm{b}}$. To obtain an optimal result for $R_{\mathrm{c}}$ various methods are employed: doubletag measurements, the counting of weakly decaying charmed hadrons (charm counting) and the detection of the leptonic decay products in the $\mathrm{D}$ meson decay chain (lepton-tag). More details on the various heavy flavour measurements performed with the experiments at LEP and SLC are given in [32] and the references therein.

Asymmetry measurements. The measurement of forwardbackward asymmetries requires the tagging of the quark flavour and the separation of quark and antiquark; a charge has to be assigned to the quark jets or hemispheres of the event. Various charge tagging methods have been employed by the experiments at LEP and SLC. They exploit the correlation between the charges of the decaying heavy quark and the 
particles in the decay chain and rely mainly on leptons, $\mathrm{D}$ mesons, jet charge, vertex charge and kaons. In every $A_{\mathrm{FB}}^{\mathrm{q} \overline{\mathrm{q}}}$ analysis the measured forward-backward asymmetry is given by

$$
A_{\mathrm{FB}}^{\mathrm{meas}}=\sum_{\mathrm{q}}\left(2 \omega_{\mathrm{q}}-1\right) \eta_{\mathrm{q}} A_{\mathrm{FB}}^{\mathrm{q} \overline{\mathrm{q}}},
$$

where $\eta_{\mathrm{q}}$ is the fraction of $\mathrm{q} \overline{\mathrm{q}}$ events in the data sample, and $\omega_{\mathrm{q}}$ is the probability to tag the quark charge correctly; the sum is taken over all quark flavours, u, d, s, c, b. It should be noted that some tagging methods tag the charge but not the flavour. In such cases the term $\left(2 \omega_{\mathrm{q}}-1\right)$ comes close to -1 for c-quarks if it is constructed to be positive for b-quarks. Similar flavour composition and quark charge tag factors apply for $A_{\mathrm{LRFB}}^{\mathrm{q} \overline{\mathrm{q}}}$ analyses.

Corrections and systematic uncertainties. The analyses of heavy quark flavour final states depend on assumptions about fragmentation and decay of the hadrons that have to be taken into account for the extraction of the $\mathrm{Z}$ pole parameters $R_{\mathrm{b}}^{0}, R_{\mathrm{c}}^{0}, A_{\mathrm{FB}}^{0, \mathrm{~b}}, A_{\mathrm{FB}}^{0, \mathrm{c}}, A_{\mathrm{LRFB}}^{\mathrm{b}}$ and $A_{\mathrm{LRFB}}^{\mathrm{c}}$ and for the combination of the results of all experiments.

Corrections to $R_{\mathrm{b}}$ and $R_{\mathrm{c}}$. Small corrections are applied to the raw measurements of the production cross section ratio, $R_{\mathrm{q}}=\sigma_{\mathrm{q} \overline{\mathrm{q}}} / \sigma_{\text {had }}$. To obtain the partial widths ratio, $R_{\mathrm{q}}^{0}=$ $\Gamma_{\mathrm{q} \overline{\mathrm{q}}} / \Gamma_{\text {had }}$ one has to correct for the small contributions from photon exchange and $\gamma-Z$ interference.

Corrections to the asymmetries. The largest corrections for the asymmetries are those for QCD effects. In contrast to the $R_{\mathrm{q}}^{0}$ measurement they are by construction not absorbed in the definition of the electroweak asymmetry parameters. The dominant QCD corrections arise from gluon radiation from the final state quarks. The QCD corrections are independent of the beam polarization and are the same for forwardbackward and left-right-forward-backward asymmetries. The QCD corrections to the asymmetry are parametrized with $\left(A_{\mathrm{FB}}^{\mathrm{q} \overline{\mathrm{q}}}\right)_{\text {meas }}=\left(1-C_{\mathrm{QCD}}\right)\left(A_{\mathrm{FB}}^{\mathrm{q} \overline{\mathrm{q}}}\right)_{\text {noQCD}}$; theoretical calculations of $C_{\mathrm{QCD}}$ depend on whether the jet axis or thrust axis is used to determine the polar angle in the analysis. The procedure to implement the QCD corrections is non-trivial; for the details see [61].

Combination of heavy flavour results. The combination of heavy flavour asymmetry results at LEP has been carried out in two steps: first, all forward-backward asymmetry measurements around the $\mathrm{Z}$ peak were averaged using $\chi^{2}$ minimization and corrected resulting in forward-backward asymmetries at three common centre-of-mass energies ('peak' and 'peak $\pm 2 \mathrm{GeV}$ ') as depicted in figure 8 . In a second step the off-peak asymmetry measurements were transferred to the 'peak' centre-of-mass energy. The sample of 'peak' asymmetries has been corrected to the $\mathrm{Z}$ pole forwardbackward asymmetries, $A_{\mathrm{FB}}^{0, \mathrm{~b}}$ and $A_{\mathrm{FB}}^{0, \mathrm{c}}$, using ZFITTER. These results have been combined with the $R_{\mathrm{b}}^{0}$ and $R_{\mathrm{c}}^{0}$ measurements as well as the $\mathcal{A}_{\mathrm{b}}$ and $\mathcal{A}_{\mathrm{c}}$ measurements at SLD. The combination procedure includes parameters correlated with

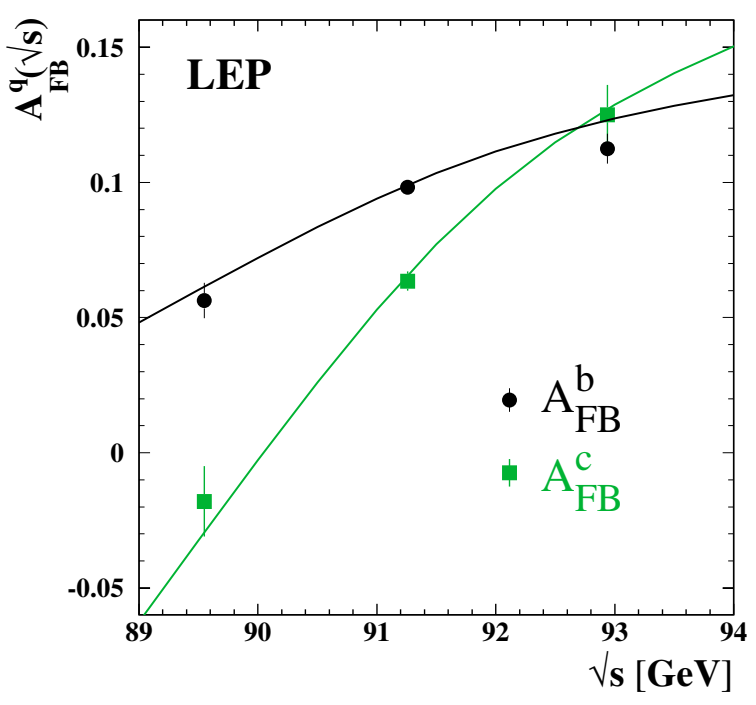

Figure 8. Energy dependence of the combined heavy flavour asymmetries.

Table 2. The results of the combination of LEP and SLD heavy flavour data [32].

\begin{tabular}{lc}
\hline Observable & Result \\
\hline$R_{\mathrm{b}}^{0}$ & $0.21629 \pm 0.00066$ \\
$R_{\mathrm{c}}^{0}$ & $0.1721 \pm 0.0030$ \\
$A_{\mathrm{FB}}^{0, \mathrm{~b}}$ & $0.0992 \pm 0.0016$ \\
$A_{\mathrm{FB}}^{0, \mathrm{c}}$ & $0.0707 \pm 0.0035$ \\
$\mathcal{A}_{\mathrm{b}}$ & $0.923 \pm 0.020$ \\
$\mathcal{A}_{\mathrm{c}}$ & $0.670 \pm 0.027$ \\
\hline
\end{tabular}

$R_{\mathrm{q}}^{0}, A_{\mathrm{FB}}^{0, \mathrm{q}}$ and $\mathcal{A}_{\mathrm{q}}$ which have been also measured by the individual experiments; the details can be found in [32]. Table 2 summarizes the results of heavy flavour measurements at the $\mathrm{Z}$ pole. An impressive accuracy of $0.3 \%$ for the $R_{\mathrm{b}}^{0}$ determination has been achieved.

\subsection{Inclusive hadronic charge asymmetry}

In addition to the inclusive hadronic $\mathrm{Z}$ pole cross section, $\sigma_{\text {had }}^{0}$, also the inclusive hadronic forward-backward charge asymmetry, $\left\langle Q_{\mathrm{FB}}\right\rangle$, averaged over all quark flavours, u,d,s,c,b, has been measured. Without separating the individual quark flavours, the charges of jets or hemispheres are assigned to the primary quark-antiquark pair

$$
\left\langle Q_{\mathrm{FB}}\right\rangle=\sum_{\mathrm{q} \neq \mathrm{t}} R_{\mathrm{q}} A_{\mathrm{FB}}^{\mathrm{q} \overline{\mathrm{q}}} \operatorname{sign}\left(Q_{\mathrm{q}}\right),
$$

where $Q_{\mathrm{q}}$ is the electric charge of quark flavour $\mathrm{q}$. Corresponding to the charges of up-type and down-type quarks, the individual flavour asymmetries, $A_{\mathrm{FB}}^{\mathrm{q} \overline{\mathrm{q}}}$, contribute with opposite signs to the hadronic forward-backward charge asymmetry. The crucial point of the $\left\langle Q_{\mathrm{FB}}\right\rangle$ measurement is the charge separation. The difference of the measured charges of the two hadronic jets reflects detector effects such as acceptance and resolution. Thus, the values for hadronic charge asymmetries measured by the individual LEP experiments cannot be combined directly. However, in the 
Standard Model the parameters $R_{\mathrm{q}}$ and $A_{\mathrm{FB}}^{\mathrm{q} \overline{\mathrm{q}}}$ can be expressed as functions of the effective weak mixing angle. Each of the LEP experiments has interpreted its $\left\langle Q_{\mathrm{FB}}\right\rangle$ measurement as value of $\sin ^{2} \theta_{\mathrm{eff}}^{\text {lept }}$ resulting in the LEP average

$$
\sin ^{2} \theta_{\mathrm{eff}}^{\text {lept }}=0.2324 \pm 0.0007 \pm 0.0010
$$

The measurement of $\left\langle Q_{\mathrm{FB}}\right\rangle$ is dominated by systematic errors. It must be emphasized that this result (equation (107)) has been obtained in the context of the Standard Model, its further use in comparisons with alternative physics models has to be considered with care.

\section{Parameters of the $Z$ boson}

The results presented in section 5 have been derived in largely model-independent analyses and are quoted in terms of experimentally motivated pseudo-observables. The $\mathrm{Z}$ boson parameters such as partial decay widths, effective vector and axial-vector coupling constants, or the effective electroweak mixing angle, can easily be derived from pseudo-observables through parameter transformations. Thus it is tested whether the $\mathrm{Z}$ boson properties are in agreement with that predicted by the Standard Model.

\subsection{The $Z$ boson mass}

The $\mathrm{Z}$ boson mass, $m_{\mathrm{Z}}=91.187 \pm 0.002 \mathrm{GeV}$, is one of the most precisely measured physical quantities. The impressive accuracy could only be achieved performing a scan of the $\mathrm{Z}$ boson resonance and the very precise measurement of the centre-of-mass energy using the method of resonant beam depolarization (see section 4). Since this method is only practicable at storage rings, the outstanding relative precision of $10^{-5}$ for the $\mathrm{Z}$ boson mass measurement will not be improved in future experiments running at the $\mathrm{Z}$ boson resonance at a linear $\mathrm{e}^{+} \mathrm{e}^{-}$collider.

In view of this excellent precision it is important to point out the role of the hadronic $\gamma-\mathrm{Z}$ interference contribution in the determination of the $\mathrm{Z}$ boson mass. According to equation (49) the contributions $\sigma_{\mathrm{Z}}, \sigma_{\gamma}$, and $\sigma_{\gamma \mathrm{Z}}$ are expressed as functions of the electromagnetic and the weak couplings. The contribution $\sigma_{\gamma}$ is determined by QED and small at the $\mathrm{Z}$ boson resonance. Also the $\gamma-\mathrm{Z}$ interference term renders only a small contribution at the $\mathrm{Z}$ boson resonance. In the case of leptonic final states, $\sigma_{\gamma z}$ is constrained by the asymmetry measurements yielding $\mathrm{Z}$ boson couplings to leptons. But exclusive asymmetry measurements do not exist for all quark flavours in the hadronic final states. Instead, the hadronic interference term can be expressed using the coupling-dependent parameter $j_{\text {tot }}^{\text {had }}$ [62-64],

$$
j_{\mathrm{tot}}^{\mathrm{had}}=\frac{G_{\mathrm{F}} m_{\mathrm{Z}}^{2}}{\sqrt{2} \pi \alpha\left(m_{\mathrm{Z}}^{2}\right)} Q_{\mathrm{e}} g_{\mathrm{Ve}} \cdot 3 \sum_{\mathrm{q} \neq \mathrm{t}} Q_{\mathrm{q}} g_{\mathrm{Vq}},
$$

taking into account inclusively the $\mathrm{Z}$ boson couplings to quarks. In the Standard Model the parameter $j_{\text {tot }}^{\text {had }}$ is $0.21 \pm 0.01$. Performing fits within the framework of the Standard Model the hadronic interference term can be fixed to that value. However, in model-independent analyses it should rather be a free parameter. In the fits presented in section $5, j_{\text {tot }}^{\text {had }}$ was fixed and it raises the question whether this procedure introduces a bias or reduces the uncertainties of the $\mathrm{Z}$ boson parameters incorrectly. The hadronic $\gamma-Z$ interference term is strongly anti-correlated with $m_{\mathrm{Z}}$, and taking it as a free parameter in a model-independent fit to the $\mathrm{Z}$ pole data enlarges the error on the $\mathrm{Z}$ boson mass by a factor of almost three [32]. The $\mathrm{Z}$ boson mass depends on $j_{\text {tot }}^{\text {had }}$,

$$
\frac{\mathrm{d} m_{\mathrm{Z}}}{\mathrm{d} j_{\mathrm{tot}}^{\mathrm{had}}}=-1.6 \mathrm{MeV} / 0.1
$$

The change in all other parameters is below $20 \%$ of their combined errors for a change of 0.1 in $j_{\text {tot }}^{\text {had }}$. Improved constraints on the hadronic interference term are given by including the measurements of hadronic cross sections far away the $\mathrm{Z}$ pole, done by the TRISTAN collaborations at KEK, TOPAZ [65] and VENUS [66] at $\sqrt{s}=58 \mathrm{GeV}$. Measurements at LEP-II [67] also constrain $j_{\text {tot }}^{\text {had }}$ and test in addition modifications of this term arising from new physics such as additional neutral gauge bosons, $Z^{\prime}$. Taking into account all available experimental constraints, the error on $j_{\text {tot }}^{\text {had }}$ is about \pm 0.1 while its central value is in good agreement with the Standard Model expectation. Thus, the uncertainties on $m_{\mathrm{Z}}$ are independent of the Standard Model assumption in the hadronic channel and no additional error has to be assigned to the $\mathrm{Z}$ boson mass.

\subsection{The Z boson partial widths}

Simultaneously with the $\mathrm{Z}$ boson mass the total $\mathrm{Z}$ boson width, $\Gamma_{\mathrm{Z}}$, and the partial widths, $\Gamma_{\mathrm{f} \overline{\mathrm{f}}}$, have been determined. The values for the partial $\mathrm{Z}$ boson widths are derived from the pseudo-observables $\sigma_{\text {had }}^{0}, R_{\mathrm{e}}^{0}, R_{\mu}^{0}, R_{\tau}^{0}$ assuming massless fermions. The partial width ratios,

$$
\begin{gathered}
\frac{\Gamma_{\mu \mu}}{\Gamma_{\mathrm{ee}}}=\frac{B\left(\mathrm{Z} \rightarrow \mu^{+} \mu^{-}\right)}{B\left(\mathrm{Z} \rightarrow \mathrm{e}^{+} \mathrm{e}^{-}\right)}=1.0009 \pm 0.0028, \\
\frac{\Gamma_{\tau \tau}}{\Gamma_{\mathrm{ee}}}=\frac{B\left(\mathrm{Z} \rightarrow \tau^{+} \tau^{-}\right)}{B\left(\mathrm{Z} \rightarrow \mathrm{e}^{+} \mathrm{e}^{-}\right)}=1.0019 \pm 0.0032
\end{gathered}
$$

are in good agreement with lepton universality. The results for the partial $\mathrm{Z}$ boson decay widths including also the heavy flavour results are summarized in table 3 [32] assuming lepton universality. The width $\Gamma_{\ell \ell}$ is that of a single charged massless lepton. The $\mathrm{Z}$ boson decay width to invisible particles is calculated as the difference of total and all other partial widths.

\subsection{The number of light neutrinos}

The decay $\mathrm{Z} \rightarrow \nu \bar{v}$ cannot be observed directly in the detector. But its appearance shows up as invisible width, $\Gamma_{\text {inv }}$,

$$
\Gamma_{\mathrm{inv}}=\Gamma_{\mathrm{Z}}-\sum_{\mathrm{e}, \mu, \tau, \mathrm{q}} \Gamma_{\mathrm{f} \overline{\mathrm{f}}} .
$$


Table 3. Partial $Z^{0}$ decay widths, derived from the results of lineshape measurements [32] assuming lepton universality.

\begin{tabular}{lc}
\hline$\Gamma_{\mathrm{f} \overline{\mathrm{f}}}$ & Average $(\mathrm{MeV})$ \\
\hline$\Gamma_{\mathrm{had}}$ & $1744.4 \pm 2.0$ \\
$\Gamma_{\ell \ell}$ & $83.985 \pm 0.086$ \\
$\Gamma_{\mathrm{b} \overline{\mathrm{b}}}$ & $377.3 \pm 1.2$ \\
$\Gamma_{\mathrm{c} \overline{\mathrm{c}}}$ & $300.2 \pm 5.2$ \\
$\Gamma_{\mathrm{inv}}$ & $499.0 \pm 1.5$ \\
\hline
\end{tabular}

The invisible width is used to determine the number of light neutrino species by assuming $\Gamma_{\text {inv }}=N_{v} \cdot \Gamma_{\nu \bar{v}}$. With

$$
N_{v}=\frac{\Gamma_{\text {inv }}}{\Gamma_{\ell \ell}}\left(\frac{\Gamma_{\ell \ell}}{\Gamma_{\nu \bar{v}}}\right)_{\mathrm{SM}}=2.9840 \pm 0.0082
$$

the number of light neutrino flavours is confirmed to be three [32]. The measurement of $\Gamma_{\text {inv }}$ is two standard deviations below the expectations from the Standard Model for three light neutrino families. The difference between measured invisible width and its predicted value is

$$
\Delta \Gamma_{\mathrm{inv}}=-2.7_{-1.5}^{+1.8} \mathrm{MeV},
$$

corresponding to a limit $\Delta \Gamma_{\text {inv }}<2.0 \mathrm{MeV}$ at the $95 \%$ confidence level for a non-standard contributions to the invisible width. The measured $\Gamma_{\text {inv }}$ could also be a hint of potentially small $\mathrm{Z} v \bar{v}$ couplings in comparison with the Standard Model expectation.

\subsection{Effective couplings of the neutral weak current}

To obtain the effective vector and axial-vector coupling constants, $g_{\text {Af }}$ and $g_{\mathrm{Vf}}$, the combination of all measurements is essential. As introduced in section 3.1, the partial widths and the width parameters, $R_{\mathrm{f}}$, depend on the sum of couplings, $g_{\mathrm{Af}}^{2}+g_{\mathrm{Vf}}^{2}$, whereas the asymmetry parameters, $\mathcal{A}_{\mathrm{f}}$, depend on the ratio, $g_{\mathrm{Vf}} / g_{\mathrm{Af}}$. Thus, the width parameters derived from cross section measurements, together with the combination of all asymmetry parameters provide quantities to disentangle $g_{\text {Af }}$ and $g_{\mathrm{Vf}}$.

Asymmetry parameters. The LEP-I and SLD results for the leptonic asymmetry parameters have been combined to obtain simultaneously results for $\mathcal{A}_{\mathrm{e}}$ and $\mathcal{A}_{\mathrm{f}}$. Besides the measurements of $\mathcal{A}_{\mathrm{e}}, \mathcal{A}_{\tau}$ from $\tau$ polarization at LEP-I and $A_{\mathrm{LR}}^{0}$ and $A_{\mathrm{LRFB}}^{0}$ at SLD, also the LEP results from leptonic forwardbackward asymmetry measurements have been included. The asymmetry parameters for the charged lepton species are summarized in the upper part of table 4, they agree well and support lepton universality. With this assumption the combined value is

$$
\mathcal{A}_{\ell}(\mathrm{LEP}-\mathrm{I}+\mathrm{SLC})=0.1501 \pm 0.0016 \text {. }
$$

The impressive accuracy of the $A_{\mathrm{LR}}^{0}$ measurement dominates the analysis and causes the small uncertainties on $\mathcal{A}_{\mathrm{e}}$ and $\mathcal{A}_{\ell}$. The results for the quark asymmetry parameters $\mathcal{A}_{\mathrm{b}}$ and $\mathcal{A}_{\mathrm{c}}$ from SLD and LEP measurements are summarized in the lower part of table 4 . The averaged leptonic asymmetry parameter $\mathcal{A}_{\ell}$ (115) has been used to derive $\mathcal{A}_{\mathrm{q}}$ from the LEP heavy flavour pole forward-backward asymmetries. Each ratio $\mathcal{A}_{\mathrm{q}}=(4 / 3) A_{\mathrm{FB}}^{0, \mathrm{q}} / \mathcal{A}_{\ell}$ determines $\mathcal{A}_{\mathrm{q}}$ with a precision comparable to that of the direct measurements but the resulting values are slightly below. This is due to the larger value of $\mathcal{A}_{\ell}$ dominated by the SLD measurements; the ratio $A_{\mathrm{FB}}^{0, \mathrm{~b}} / A_{\mathrm{FB}}^{0, \mathrm{c}}$ measured at LEP agrees well with the ratio $\mathcal{A}_{\mathrm{b}} / \mathcal{A}_{\mathrm{c}}$ measured at SLD.

The mutual consistency of the heavy flavour measurements at LEP and SLC is illustrated in figure 9. Bands of \pm 1 standard deviation width are shown in the $\left(\mathcal{A}_{\ell}, \mathcal{A}_{\mathrm{q}}\right)$ plane for the measurements of $\mathcal{A}_{\ell}, \mathcal{A}_{\mathrm{q}}$ and $A_{\mathrm{FB}}^{0, \mathrm{q}}=(3 / 4) \mathcal{A}_{\mathrm{e}} \mathcal{A}_{\mathrm{q}}$. The $68 \%, 95 \%$ and $99.5 \%$ confidence level contours for the two asymmetry parameters resulting from the joint analysis (table 4 , last row) are also depicted. Since $A_{\mathrm{FB}}^{0, \mathrm{q}}$ and $\mathcal{A}_{\ell}$ are measured with small errors, the joint analysis improves primarily the error on $\mathcal{A}_{\mathrm{q}}$ and tends to pull the asymmetry parameter $\mathcal{A}_{\ell}$ to smaller values. For comparison, also the Standard Model expectations for $\mathcal{A}_{\mathrm{q}}$ and $\mathcal{A}_{\ell}$ are depicted in figure $9 ; \mathcal{A}_{\mathrm{b}}$ from the $A_{\mathrm{FB}}^{0, \mathrm{~b}}$ measurement is three standard deviations below. It should be noted that although substantially higher statistics of $\mathrm{Z}$ boson events at LEP, the precision of the $\mathcal{A}_{\mathrm{f}}$ measurements at SLC is competitive or higher due to the direct measurement of the asymmetry parameters and the high polarization provided by SLC.

Effective $Z$ boson coupling to leptons and quarks. The asymmetry parameters $\mathcal{A}_{\mathrm{f}}$ depend on the ratio $g_{\mathrm{Vf}} / g_{\mathrm{Af}}$ of the effective vector and axial-vector coupling constants, the relative sign of the couplings $g_{\mathrm{Vf}}$ and $g_{\mathrm{Af}}$ is determined by $\mathcal{A}_{\mathrm{f}}$. The partial $\mathrm{Z}$ boson widths depend on the sum of the squares of these couplings. Both, partial widths and $\mathcal{A}_{\mathrm{f}}$ are invariant under the exchange $g_{\mathrm{Af}} \leftrightarrow g_{\mathrm{Vf}}$. This ambiguity can be resolved if the energy dependence of the forwardbackward asymmetries measured at LEP is exploited and both, partial widths and s-dependent asymmetry measurements are simultaneously included in the analysis. The sign of the couplings is defined by the convention $g_{\mathrm{Ae}}<0$.

The $\mathrm{Z}$ pole asymmetry data measured at LEP and SLD have been used to derive values for the effective couplings, $g_{\mathrm{Vf}}$ and $g_{\mathrm{Af}}$, of the charged leptons and heavy quarks. The results for the leptonic couplings are depicted in figure 10. The contours in the $\left(g_{\mathrm{A} \ell}, g_{\mathrm{V} \ell}\right)$ plane illustrate the consistency among the lepton flavours and show also the agreement with the Standard Model expectations. The averaged leptonic axial-vector coupling, $g_{\mathrm{A} \ell}=-0.50123 \pm 0.00026$, deviates from the Born level expectation $g_{\mathrm{A}}^{\text {tree }}=0.5$ by 4.8 standard deviations and indicates the presence of electroweak corrections.

Including the measurements of heavy quark final states, the effective couplings of the $\mathrm{Z}$ boson to leptons and quarks have been derived simultaneously assuming lepton universality. The resulting contours in the $\left(g_{\mathrm{Aq}}, g_{\mathrm{Vq}}\right)$ plane are shown in figure 11, together with the Standard Model expectations. The difference between measured b-quark couplings and predictions are due to the values for the leptonic couplings as discussed above for the $\mathcal{A}_{\mathrm{b}}-\mathcal{A}_{\ell}$ contours. 
Table 4. Results on the asymmetry parameters [32]. The leptonic asymmetry parameters are summarized in the upper part of the table. The results for the individual measurements as well as the combination are shown. In the lower part the quark asymmetry parameters are given. The results for $\mathcal{A}_{\mathrm{q}}$ have been derived using the averaged $\mathcal{A}_{\ell}$.

\begin{tabular}{lllcl}
\hline Parameter & $A_{\mathrm{FB}}^{0, \mathrm{f}}$ & $\mathcal{P}_{\tau}$ & $A_{\mathrm{LR}}^{0}, A_{\mathrm{LRFB}}^{0}$ & Average \\
\hline $\mathcal{A}_{\mathrm{e}}$ & $0.139 \pm 0.012$ & $0.1498 \pm 0.0048$ & $0.1516 \pm 0.0021$ & $0.1514 \pm 0.0019$ \\
$\mathcal{A}_{\mu}$ & $0.162 \pm 0.019$ & & $0.142 \pm 0.015$ & $0.1456 \pm 0.0091$ \\
$\mathcal{A}_{\tau}$ & $0.180 \pm 0.023$ & $0.1439 \pm 0.0043$ & $0.136 \pm 0.015$ & $0.1449 \pm 0.0040$ \\
$\mathcal{A}_{\ell}$ & & & & $0.1501 \pm 0.0016$ \\
$\mathcal{A}_{\mathrm{b}}$ & $0.881 \pm 0.017$ & & $0.923 \pm 0.020$ & $0.899 \pm 0.013$ \\
$\mathcal{A}_{\mathrm{c}}$ & $0.628 \pm 0.032$ & & $0.670 \pm 0.027$ & $0.654 \pm 0.021$ \\
\hline
\end{tabular}
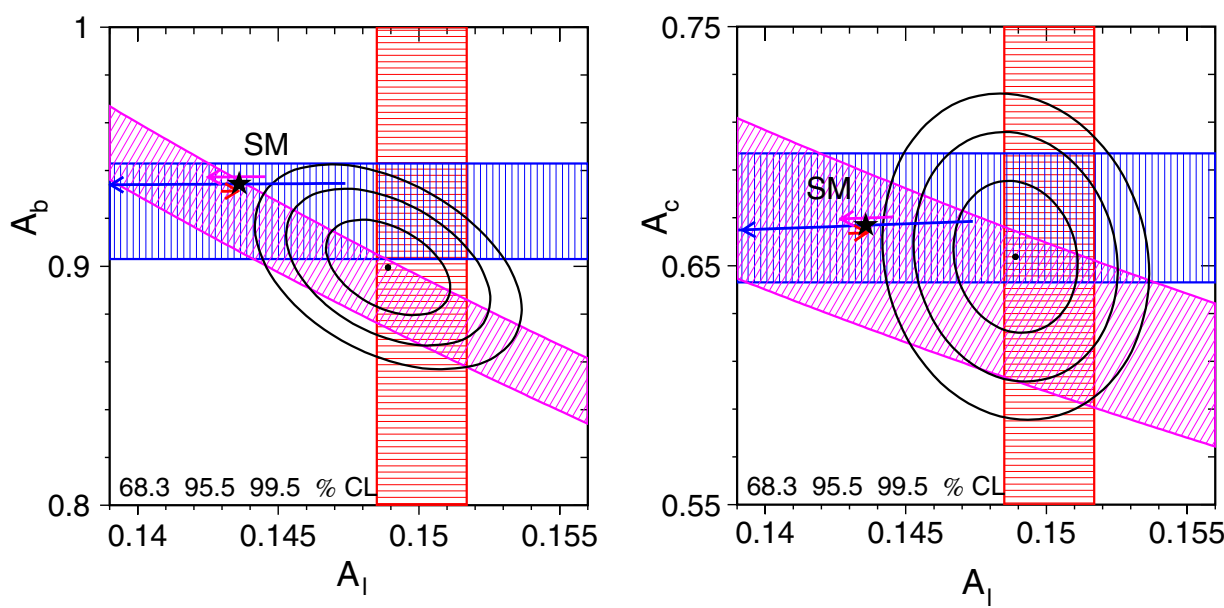

Figure 9. Comparison of the measurements of $\mathcal{A}_{\ell}, \mathcal{A}_{\mathrm{q}}$ and $A_{\mathrm{FB}}^{0, \mathrm{q}}$ for (left) b-quarks, and (right) c-quarks, assuming lepton universality [32]. Bands of \pm 1 standard deviation width in the $\left(\mathcal{A}_{\ell}, \mathcal{A}_{\mathrm{q}}\right)$ plane are shown for the measurements of $\mathcal{A}_{\ell}$ (vertical band), $\mathcal{A}_{\mathrm{q}}$ (horizontal band) and $A_{\mathrm{FB}}^{0, \mathrm{q}}=(3 / 4) \mathcal{A}_{\mathrm{e}} \mathcal{A}_{\mathrm{q}}$ (diagonal band). Also shown are the $68 \%, 95 \%$ and $99.5 \%$ confidence level contours for the two asymmetry parameters resulting from the joint analysis (table 4). The arrows pointing to the right and to the left show the variation in the SM prediction for varying $\Delta \alpha_{\text {had }}^{(5)}\left(m_{\mathrm{Z}}^{2}\right)$ in the range $0.02758 \pm 0.00035$ (arrow displaced vertically upwards), $m_{\mathrm{H}}$ in the range of $300_{-186}^{+700} \mathrm{GeV}$, and $m_{\mathrm{t}}$ in the range $173.3 \pm 1.1 \mathrm{GeV}$ (arrow displaced vertically downwards). All arrows point in the direction of increasing values of these parameters.

\subsection{The effective weak mixing angle}

The effective Zf $\bar{f}$ couplings are related to the effective electroweak mixing angle. In the Standard Model they are determined by the charge and isospin assignments:

$$
\frac{g_{\mathrm{Vf}}}{g_{\mathrm{Af}}}=1-\frac{2 Q_{\mathrm{f}}}{T_{3}^{\mathrm{f}}} \sin ^{2} \theta_{\text {eff }}^{\mathrm{f}}=1-4\left|Q_{\mathrm{f}}\right| \sin ^{2} \theta_{\text {eff }}^{\mathrm{f}} .
$$

It is obvious that the asymmetry parameters, $\mathcal{A}_{\mathrm{f}}$, are most sensitive to the effective weak mixing angle. Compared with quarks the coupling parameters for leptons depend stronger on the weak mixing angle, and the asymmetry parameters for quarks are rather insensitive to $\sin ^{2} \theta_{\text {eff }}^{\mathrm{f}}$. Hence, the effective weak mixing angle, $\sin ^{2} \theta_{\text {eff }}^{\mathrm{f}}$, has been determined analysing all observables that depend on $\mathcal{A}_{\ell}$ : the results for $A_{\mathrm{FB}}^{0, \ell}, \mathcal{A}_{\ell}\left(\mathcal{P}_{\tau}\right)$ and $\mathcal{A}_{\ell}(\mathrm{SLD})$ have been combined with the heavy flavour results, $A_{\mathrm{FB}}^{0, \mathrm{~b}}, A_{\mathrm{FB}}^{0, \mathrm{c}}$, and with $Q_{\mathrm{FB}}^{\text {had }}$, to derive the leptonic weak mixing angle. Small non-universal flavour corrections, making $\sin ^{2} \theta_{\text {eff }}^{\text {lept }}$ different from $\sin ^{2} \theta_{\text {eff }}^{\mathrm{q}}$, have been taken from the Standard Model. The average is

$$
\sin ^{2} \theta_{\mathrm{eff}}^{\text {lept }}=0.23153 \pm 0.00016,
$$

with a $\chi^{2} /$ dof of $11.8 / 5$, corresponding to a probability of only $3.7 \%$. The reason is the difference between the precise measurement of $\mathcal{A}_{\ell}$ which is dominated by the $A_{\mathrm{LR}}^{0}$ result from SLD and the asymmetry measurement at LEP, $A_{\mathrm{FB}}^{0, \mathrm{~b}}$. The results of these measurements differ in opposite directions from the average and reflect the tension between Standard Model expectation and $\mathcal{A}_{\mathrm{b}}$ discussed above. The individual results for $\sin ^{2} \theta_{\text {eff }}^{\text {lept }}$ and the average are summarized in figure 12 .

\subsection{Sensitivity to radiative corrections beyond QED}

It is a fundamental question whether the $\mathrm{Z}$ pole measurements confirm the existence of electroweak radiative corrections beyond those predicted by the theory of QED. Including only the running of the electromagnetic coupling constant, the expectation for the $\rho$ parameter and the electroweak mixing parameter are given in section 2 , by setting $\Delta \rho=\Delta \kappa=$ $\Delta r_{\mathrm{w}}=0$. This results in

$$
\begin{aligned}
& \rho_{0}=1, \\
& \sin ^{2} \theta_{0}=\frac{1}{2}\left(1-\sqrt{1-4 \frac{\pi \alpha\left(m_{\mathrm{Z}}^{2}\right)}{\sqrt{2} G_{\mathrm{F}} m_{\mathrm{Z}}^{2}}}\right) \\
& =0.23098 \pm 0.00012,
\end{aligned}
$$

where the uncertainty on $\sin ^{2} \theta_{0}$ arises from the uncertainty on $\alpha\left(m_{\mathrm{Z}}^{2}\right)$ mainly due to the hadronic vacuum polarization $\Delta \alpha_{\text {had }}^{(5)}\left(m_{Z}^{2}\right)$ (see section 7.1). 


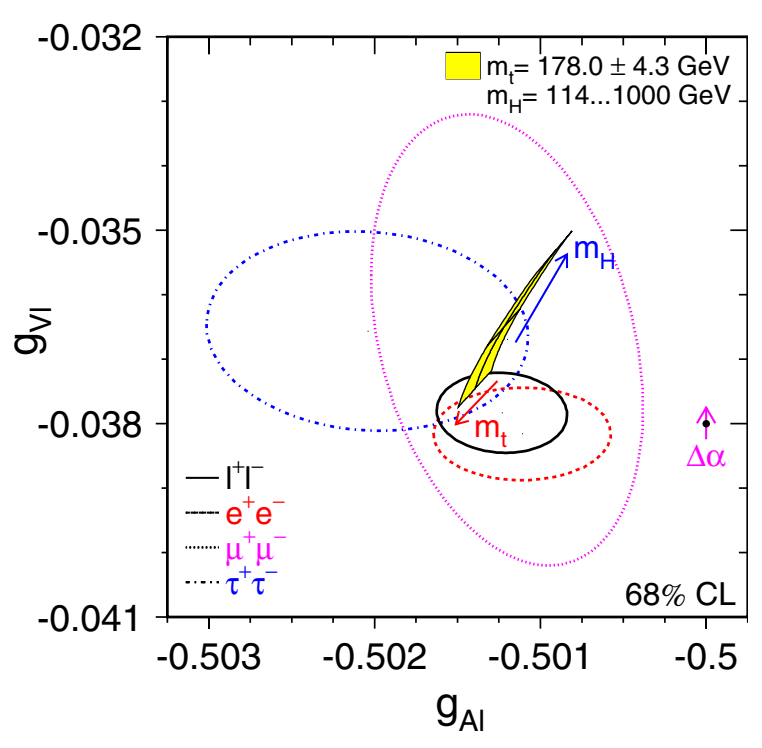

Figure 10. Comparison of the effective vector and axial-vector coupling constants for leptons [32]. The shaded region in the lepton plot shows the predictions within the SM for $m_{\mathrm{t}}=178.0 \pm 4.3 \mathrm{GeV}$ and $m_{\mathrm{H}}=300_{-186}^{+700} \mathrm{GeV}$; varying the hadronic vacuum polarization by $\Delta \alpha_{\text {had }}^{(5)}\left(m_{\mathrm{Z}}^{2}\right)=0.02758 \pm 0.00035$ yields an additional uncertainty on the SM prediction shown by the arrow labelled $\Delta \alpha$. (Reprinted with permission from [32]. Copyright 2006 Elsevier.)

The measured values for $\rho_{\ell}$ and $\sin ^{2} \theta_{\text {eff }}^{\text {lept }}$ are

$$
\begin{gathered}
\rho_{\ell}=1.0050 \pm 0.0010, \\
\sin ^{2} \theta_{\text {eff }}^{\text {lept }}=0.23153 \pm 0.00016,
\end{gathered}
$$

and they differ significantly from the predictions (118) and (119). This result indicates that electroweak corrections beyond QED are needed to describe the measurements on the $\mathrm{Z}$ pole. Figure 13 illustrates this showing in the $\left(\rho_{\ell}, \sin ^{2} \theta_{\text {eff }}^{\text {lept }}\right)$ plane the measured values together with the prediction from a theory based on electroweak Born-level formulae including QED.

\section{Global test of the Standard Model}

The consistency of the Standard Model framework requires that all measurements are accommodated by the same values of the input parameters (46). The LEP and SLD measurements at the $\mathrm{Z}$ pole are now comprised into a sample of pseudoobservables given in tables 1 and 2, and equations (93), (98) and (107). From this sample the five Standard Model parameters, $m_{\mathrm{Z}}, m_{\mathrm{t}}, m_{\mathrm{H}}, \alpha\left(m_{\mathrm{Z}}^{2}\right), \alpha_{\mathrm{S}}\left(m_{\mathrm{Z}}^{2}\right)$, have been determined in a global $\chi^{2}$ fit. The uncertainty for $\alpha\left(m_{\mathrm{Z}}^{2}\right)$ is determined by $\Delta \alpha_{\text {had }}^{(5)}\left(m_{\mathrm{Z}}^{2}\right)$, hence the latter is used instead of $\alpha$ as free parameter in the electroweak fit; its treatment is discussed in the next subsection. The $\mathrm{Z}$ boson mass derived in the modelindependent analyses presented in section 5 is now an input parameter for the calculation of observables based on the Standard Model, the underlying theory.

A comprehensive test of the Standard Model requires that also results from other experiments and their implications on the Standard Model parameters have to be included.
Consistent results provide additional constraints to Standard Model parameters, in particular to the Higgs boson mass. This global test of the Standard Model proceeds in several steps:

(i) The first step is a Standard Model fit to the Z pole data.

(ii) $\mathrm{Z}$ pole data predict the top quark mass. At the Tevatron the top quark mass has been measured directly with the CDF and $\mathrm{D} \emptyset$ detectors; these results constrain the top mass in further fits of Standard Model parameters.

(iii) Results from $\mathrm{Z}$ pole data determine the $\mathrm{W}$ boson mass and width [32]. A comparison with direct $\mathrm{W}$ boson mass measurements at LEP-II and at the Tevatron affords a global test of the Standard Model.

(iv) The electroweak sector of the Standard Model is also probed by observing parity violation at low energies. The effective weak coupling constants are measured and compared with the results at high energies taking into account the running of the coupling constants with $Q^{2}$.

(v) The Standard Model predicts cross sections and asymmetry measurements at energies above the $\mathrm{Z}$ pole. Differences between measurements and expectations could indicate new physics phenomena beyond the Standard Model or exclude them up to a certain level (see section 8.2).

In this section the results for steps (i)-(iv) are presented. The measurement of the top quark mass at the Tevatron, of the $\mathrm{W}$ boson mass and width at LEP and the Tevatron, and the electroweak results from experiments at low energies are used to constrain the electroweak parameters.

\subsection{Hadronic vacuum polarization}

As discussed in section 2.2, the running of the electromagnetic coupling with the momentum transfer, $\alpha(0) \rightarrow \alpha(s)$, due to leptonic and hadronic loop insertions into the photon propagator is described by

$$
\alpha(s)=\frac{\alpha(0)}{1-\Delta \alpha_{\text {lept }}(s)-\Delta \alpha_{\text {top }}(s)-\Delta \alpha_{\text {had }}^{(5)}(s)} .
$$

The contribution $\Delta \alpha_{\text {lept }}$ is known to third order with negligible uncertainty [68]. The top quark contribution, $\Delta \alpha_{\text {top }}$, is small because heavy particles decouple in QED, and $\Delta \alpha_{\text {top }} \simeq$ $-0.00007(1)$ is specified using TOPAZ0 and ZFITTER. However, for the light hadronic part, $\Delta \alpha_{\text {had }}^{(5)}(s)$, perturbative QCD is not applicable and quark masses are no reasonable input parameters. This term is best obtained by analysing lowenergy $\mathrm{e}^{+} \mathrm{e}^{-}$data using a dispersion relation,

$\Delta \alpha_{\text {had }}^{(5)}(s)=-\frac{\alpha(0)}{3 \pi} m_{\mathrm{Z}}^{2} \operatorname{Re} \int_{4 m_{\pi}^{2}}^{\infty} \mathrm{d} s^{\prime} \frac{R^{\gamma}\left(s^{\prime}\right)}{s^{\prime}\left(s^{\prime}-m_{\mathrm{Z}}^{2}-i \epsilon\right)}$

with

$$
R^{\gamma}\left(s^{\prime}\right)=\frac{\sigma\left(\mathrm{e}^{+} \mathrm{e}^{-} \rightarrow \gamma^{\star} \rightarrow \text { hadrons }\right)}{\sigma\left(\mathrm{e}^{+} \mathrm{e}^{-} \rightarrow \gamma^{\star} \rightarrow \mu^{+} \mu^{-}\right)} .
$$

The hadronic vacuum polarization $\Delta \alpha_{\text {had }}^{(5)}\left(m_{\mathrm{Z}}^{2}\right)$ has been determined in dedicated analyses of hadronic cross sections measured at low centre-of-mass energies [69-71], or using also theory constraints introducing some model dependence but leading to higher precision $[72,73]$. The experimentally 

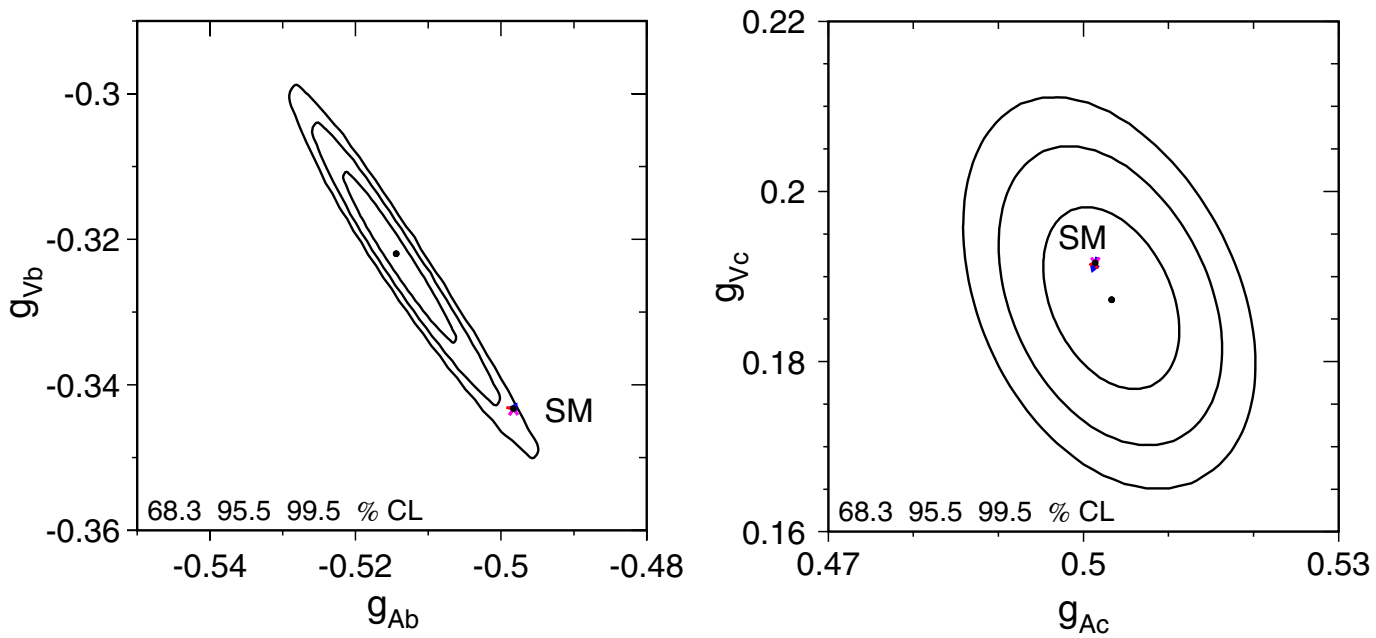

Figure 11. Comparison of the effective vector and axial-vector coupling constants for heavy quarks, using results on leptons and assuming lepton universality [32]. Left: b-quarks. Right: c-quarks. Compared with the experimental uncertainties, the SM predictions for the heavy quarks $b$ and $\mathrm{c}$ have negligible dependence on the SM input parameters. (Reprinted with permission from [32]. Copyright 2006 Elsevier.)

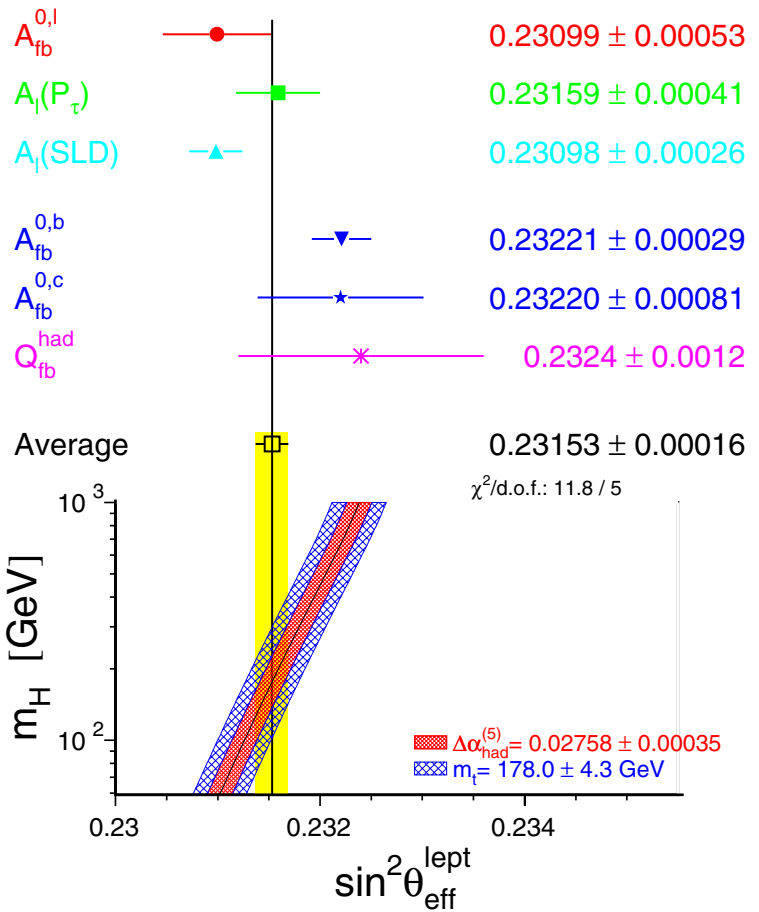

Figure 12. Comparison of the effective electroweak mixing angle $\sin ^{2} \theta_{\text {eff }}^{\text {lept }}$ derived from measurements depending on lepton couplings only and also on quark couplings [32]. Also shown is the SM prediction for $\sin ^{2} \theta_{\text {eff }}^{\text {lept }}$ as a function of $m_{\mathrm{H}}$. The additional uncertainty of the SM prediction is parametric and dominated by the uncertainties in $m_{\mathrm{t}}$ and the hadronic vacuum polarization,

$\Delta \alpha_{\text {had }}^{(5)}\left(m_{\mathrm{Z}}^{2}\right)$, shown as the band. The width of the band is the linear sum of these effects. (Reprinted with permission from [32]. Copyright 2006 Elsevier.)

driven result [71] is

$$
\Delta \alpha_{\text {had }}^{(5)}\left(m_{\mathrm{Z}}^{2}\right)=0.02758 \pm 0.00035,
$$

the more precise theory-driven result [73] gives

$$
\Delta \alpha_{\text {had }}^{(5)}\left(m_{\mathrm{Z}}^{2}\right)=0.02749 \pm 0.00012 .
$$

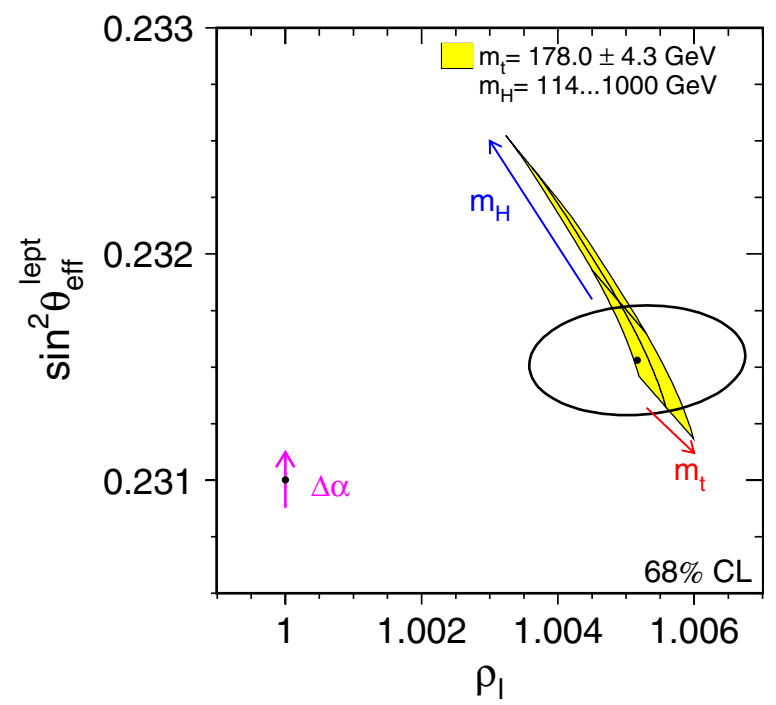

Figure 13. Contour curve of $68 \%$ probability in the $\left(\rho_{\ell}, \sin ^{2} \theta_{\text {eff }}^{\text {lept }}\right)$ plane [32]. The prediction of a theory based on electroweak Born-level formulae and QED with running $\alpha$ is shown as the dot, with the arrow representing the uncertainty due to the hadronic vacuum polarization $\Delta \alpha_{\text {had }}^{(5)}\left(m_{\mathrm{Z}}^{2}\right)$. The same uncertainty also affects the SM prediction, shown as the shaded region drawn for fixed $\Delta \alpha_{\text {had }}^{(5)}\left(m_{\mathrm{Z}}^{2}\right)$ while $m_{\mathrm{t}}$ and $m_{\mathrm{H}}$ are varied in the ranges indicated. (Reprinted with permission from [32]. Copyright 2006 Elsevier.)

For the analyses the experimentally driven value given in equation (125) has been used.

\subsection{Fit to Z pole data}

The relevant Standard Model input parameters, $m_{\mathrm{Z}}, m_{\mathrm{t}}$, $m_{\mathrm{H}}, \Delta \alpha_{\text {had }}^{(5)}\left(m_{\mathrm{Z}}^{2}\right), \alpha_{\mathrm{S}}\left(m_{\mathrm{Z}}^{2}\right)$, have been determined with $\chi^{2}$ minimization using the program MINUIT [74]. The $\chi^{2}$ has been calculated by comparing the measured $\mathrm{Z}$ pole observables ( $m_{\mathrm{Z}}, \Gamma_{\mathrm{Z}}, \sigma_{\text {had }}^{0}, R_{\ell}^{0}, A_{\mathrm{FB}}^{0, \ell}, \mathcal{A}_{\ell}, R_{\mathrm{q}}, A_{\mathrm{FB}}^{0, \mathrm{q}}, A_{\mathrm{FB}}^{0, \mathrm{c}}, \mathcal{A}_{\mathrm{q}}$ ) including their errors and correlations with the predictions in the framework of the Standard Model. 
Table 5. Results [32] for the five SM input parameters derived from a fit to the $\mathrm{Z}$ pole results and the hadronic vacuum polarization, $\Delta \alpha_{\text {had }}^{(5)}\left(m_{\mathrm{Z}}^{2}\right)$. The results on $m_{\mathrm{H}}$ are also shown.

\begin{tabular}{lccrlrl}
\hline & & \multicolumn{5}{c}{ Correlations } \\
\cline { 3 - 7 } Parameter & \multicolumn{1}{c}{ Value } & $\Delta \alpha_{\text {had }}^{(5)}\left(m_{\mathrm{Z}}^{2}\right)$ & $\alpha_{\mathrm{S}}\left(m_{\mathrm{Z}}^{2}\right)$ & $m_{\mathrm{Z}}$ & $m_{\mathrm{t}}$ & $\log _{10} \frac{m_{\mathrm{H}}}{\mathrm{GeV}}$ \\
\hline$\Delta \alpha_{\mathrm{had}}^{(5)}\left(m_{\mathrm{Z}}^{2}\right)$ & $0.02759 \pm 0.00035$ & 1.00 & & & & \\
$\alpha_{\mathrm{S}}\left(m_{\mathrm{Z}}^{2}\right)$ & $0.1190 \pm 0.0027$ & -0.04 & 1.00 & & & \\
$m_{\mathrm{Z}}(\mathrm{GeV})$ & $91.1874 \pm 0.0021$ & -0.01 & -0.03 & 1.00 & & \\
$m_{\mathrm{t}}(\mathrm{GeV})$ & $173 \pm_{10}^{13}$ & -0.03 & 0.19 & -0.07 & 1.00 & \\
$\log _{10} \frac{m_{\mathrm{H}}}{\mathrm{GeV}}$ & $2.05 \pm_{0.34}^{0.43}$ & -0.29 & 0.25 & -0.02 & 0.89 & 1.00 \\
$m_{\mathrm{H}}(\mathrm{GeV})$ & $111 \pm_{60}^{190}$ & -0.29 & 0.25 & -0.02 & 0.89 & 1.00 \\
\hline
\end{tabular}

In contrast to the procedure in section 5.3 deriving the $\mathrm{Z}$ boson mass in a model-independent manner by a fit to the $\mathrm{Z}$ lineshape data, $m_{\mathrm{Z}}$ is here an input parameter that enters all observables directly and also via electroweak loop corrections. The top quark and Higgs boson masses also contribute to the loop corrections (see section 2.1) and have been derived from the fit assuming the validity of the Standard Model. The fit results and their correlations are summarized in table 5. It should be noted that the $\chi^{2}$ for the fit is $16 / 10$ corresponding to a p-value of $9.9 \%$. The data measured at the $\mathrm{Z}$ pole have only low sensitivity to the hadronic vacuum polarization, $\Delta \alpha_{\text {had }}^{(5)}\left(m_{Z}^{2}\right)$. But this parameter is largely correlated with the Higgs boson mass and therefore important for the determination of $m_{\mathrm{H}}$. Although the Higgs boson mass enters the electroweak corrections only logarithmically, the excellent precision of the $\mathrm{Z}$ pole data yields an impressive constraint on $m_{\mathrm{H}}$. However, the fit results for the Higgs boson mass and the top quark mass are highly correlated as can be seen in table 5. The electroweak radiative corrections depend on both top quark mass and Higgs boson mass as described in section 2.2. Hence, constraining $m_{\mathrm{t}}$ in further fits with the value resulting from direct measurements of the top quark mass, also the Higgs boson mass is specified. The fit result for the strong coupling constant, $\alpha_{\mathrm{S}}\left(m_{\mathrm{Z}}^{2}\right)$, is in good agreement with other determinations $[9,75,76]$. It is mainly determined by the ratio $R_{\ell}^{0}=\Gamma_{\text {had }} / \Gamma_{\ell \ell}$ which is sensitive to QCD corrections in the hadronic width described by the factor $R_{\mathrm{A}, \mathrm{QCD}}$ given in equation (60).

The Standard Model parameters obtained from measurements at the $\mathrm{Z}$ pole determine a value for the $\mathrm{W}$ boson mass

$$
m_{\mathrm{W}}=80.363 \pm 0.032 \mathrm{GeV} .
$$

Performing a consistency test of the Standard Model, this W boson mass as well as the top quark mass have to be compared with the result from direct measurements.

\subsection{W boson production at LEP-II}

The production of $\mathrm{W}$ boson pairs, $\mathrm{e}^{+} \mathrm{e}^{-} \rightarrow \mathrm{W}^{+} \mathrm{W}^{-}$, has been studied by the LEP experiments since 1996 when the kinematic threshold of $\sqrt{s}=2 m_{\mathrm{W}}$ was reached. Above the threshold, on-shell $\mathrm{W}$ boson pairs are created; the Feynman diagrams contributing to this process in the Born approximation are shown in figure 14. The couplings among the gauge bosons are given by the structure of the gauge group. Thus, this gauge structure can be directly determined by the measurement of gauge boson interaction in W-pair production. Of special interest are the $s$-channel diagrams which are sensitive to the triple-gauge-boson vertex of interaction between neutral and charged gauge bosons of electroweak interactions. Without gauge interactions, $\mathrm{W}^{+} \mathrm{W}^{-}$pairs are produced in $\mathrm{e}^{+} \mathrm{e}^{-}$ collisions via neutrino $t$-channel exchange (see left plot in figure 14). This mechanism violates unitarity at high energies and is regulated by the photon and $\mathrm{Z}$ boson $s$-channel exchange processes. To restore unitarity, the exact values of the self-couplings, as predicted by the $\mathrm{SU}(2)_{\mathrm{L}} \times \mathrm{U}(1)_{\mathrm{Y}}$ gauge structure, are needed; small deviations lead to large variations of the cross section at high energies. For this reason, the process $\mathrm{e}^{+} \mathrm{e}^{-} \rightarrow \mathrm{W}^{+} \mathrm{W}^{-}$is very sensitive to the triple gauge boson couplings.

W boson pairs are detected by their decays to four-fermion final states: via charged weak currents the $\mathrm{W}$ bosons decay to leptons or hadrons. For the event selection of $\mathrm{W}$ boson pairs three different topologies have been distinguished: pure leptonic final states, $\mathrm{W}^{+} \mathrm{W}^{-} \rightarrow \ell \bar{v}_{\ell} \ell \bar{v}_{\ell}$, which contribute about $11 \%$, semileptonic $\mathrm{W}^{+} \mathrm{W}^{-} \rightarrow \mathrm{q} \overline{\mathrm{q}} \ell v_{\ell}(44 \%)$ and fully hadronic final states, $\mathrm{W}^{+} \mathrm{W}^{-} \rightarrow \mathrm{q} \overline{\mathrm{q}} \mathrm{q} \overline{\mathrm{q}}(45 \%)$. The leptonic final states can be identified with high efficiency and low background contributions from other processes but the separation of fully hadronic events from background due to QCD four-jet production is more complex.

The $\mathrm{W}$ boson measurements performed during the LEP-II phase ranged in centre-of-mass energies from $161 \mathrm{GeV}$ up to $209 \mathrm{GeV}$ and each of the LEP experiments collected about $700 \mathrm{fb}^{-1}$ of integrated luminosity. In total, this corresponds to a data sample of approximately $40000 \mathrm{~W}$ boson pairs produced at LEP-II. The average cross section measured at LEP-II as a function of the centre-of-mass energy is shown in figure 15 . The measurements agree with the Standard Model predictions and rule out hypotheses of non-existing ZWW vertex or $t$-channel exchange of electron neutrinos only. Thus, the existence of triple gauge boson couplings has been confirmed experimentally. The $\mathrm{W}^{+} \mathrm{W}^{-}$cross sections measured for each final state allow a simultaneous determination of total cross section and branching fractions of the $\mathrm{W}$ boson decay.

Determination of $W$ boson mass and width at LEP-II. The first measurements of the $\mathrm{W}$ boson mass at LEP-II were 


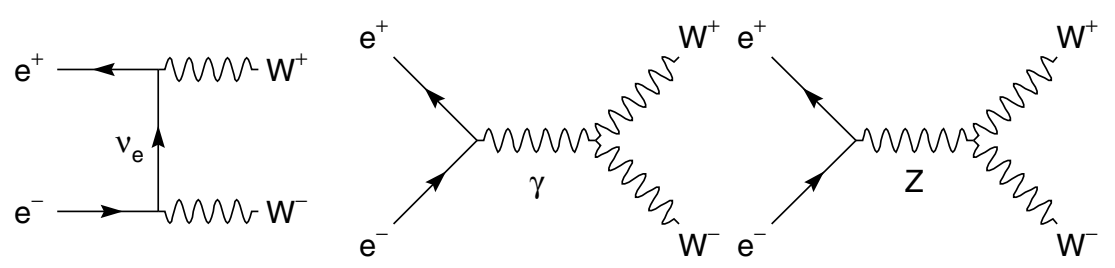

Figure 14. Feynman diagrams of the process $\mathrm{e}^{+} \mathrm{e}^{-} \rightarrow \mathrm{W}^{+} \mathrm{W}^{-}$in lowest order.

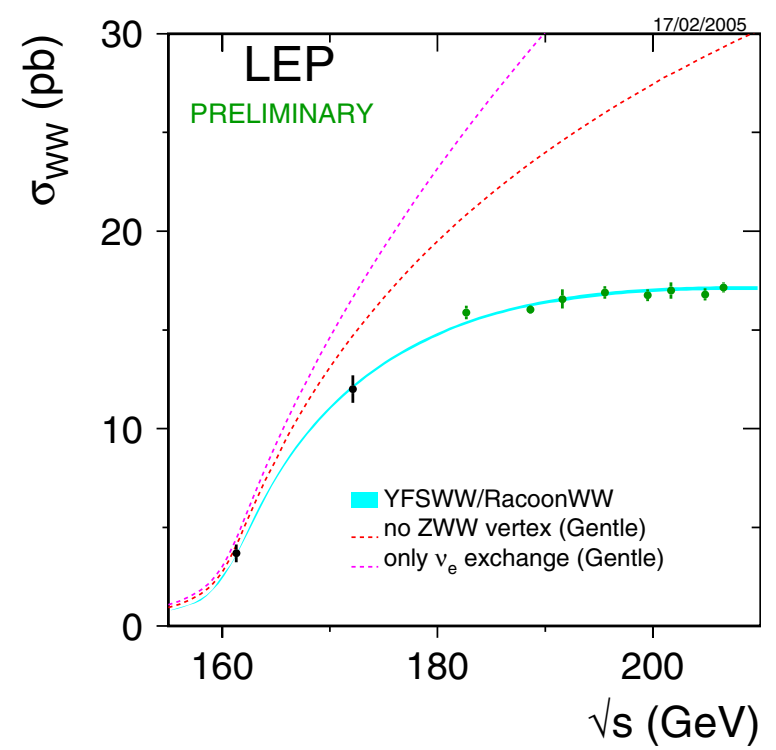

Figure 15. The $\mathrm{W}^{+} \mathrm{W}^{-}$pair cross sections measured at LEP-II compared with predictions for theories without the $\gamma \mathrm{WW}$ and ZWW vertices.

performed at the $\mathrm{W}$-pair production threshold, $\sqrt{s} \simeq 161 \mathrm{GeV}$, see [82-85] where the $\mathrm{W}$-pair cross section as a function of centre-of-mass energy depends strongly on the $\mathrm{W}$ boson mass. But the main part of the W-pair events was collected at higher energies where the cross section is less dependent on $m_{\mathrm{W}}$; so the $\mathrm{W}$ boson mass has been determined directly by reconstruction of $\mathrm{W}$-pair events. The signature of $\mathrm{W}$-pairs decaying to four fermions was reconstructed in the detector, energy and direction of the fermions were paired to rebuild the $\mathrm{W}$ bosons: the invariant mass of the fermion system corresponds to the invariant mass of the $\mathrm{W}$ boson. Imposing energy and momentum conservation as well as equal masses for the $\mathrm{W}$ bosons in a kinematic fit for each event improves the mass resolution substantially. The energy scale for the $\mathrm{W}$ boson is determined by the beam energy measurement. As already discussed in section 4.2, at LEP-II the beam energy was derived from dipole fields measured with NMR probes, and the calibration was performed using the resonant depolarization method in special runs at the $\mathrm{Z}$ pole. This procedure has yielded an uncertainty of $\Delta m_{\mathrm{W}}= \pm 17 \mathrm{MeV}$ common to all LEP experiments. The spectra of reconstructed invariant masses show the peak of the $\mathrm{W}$ boson mass around $80 \mathrm{GeV}$. The mass and total decay width of the $\mathrm{W}$ boson have been extracted by analysing these distributions assuming a BreitWigner form with $s$-dependent decay width and taking into account the detector resolution.
A particular problem of $\mathrm{W}$ boson mass measurements arises for pure hadronic final states, $\mathrm{W}^{+} \mathrm{W}^{-} \rightarrow \mathrm{q} \overline{\mathrm{q}} \mathrm{q} \overline{\mathrm{q}}$, between the decay products of the two different $\mathrm{W}$ bosons so-called final state interactions (FSI) are possible: colour reconnection (CR) and Bose-Einstein correlations (BEC).

Colour reconnection. The typical distance between the decay points of the two $\mathrm{W}$ bosons, approximately $0.1 \mathrm{fm}$, is much smaller than the hadronization scale of the quarks, which is $1 \mathrm{fm}$. So, gluons are exchanged between quarks from different $\mathrm{W}$ bosons implying a momentum transfer and hence, a bias in the measurement of the invariant mass. In particular, soft gluons with energies $E_{\mathrm{g}}<\Gamma_{W}$ may have a large impact on the reconstructed $\mathrm{W}$ boson mass. This colour reconnection has been investigated by comparing the charged particle multiplicity in $\mathrm{W}^{+} \mathrm{W}^{-} \rightarrow \mathrm{q} \overline{\mathrm{q}} \mathrm{q} \overline{\mathrm{q}}$ and $\mathrm{W}^{+} \mathrm{W}^{-} \rightarrow \mathrm{q} \overline{\mathrm{q}} \ell \nu_{\ell}$ events and by comparing the particle flow in $\mathrm{W}^{+} \mathrm{W}^{-} \rightarrow \mathrm{q} \overline{\mathrm{q}} \mathrm{q} \overline{\mathrm{q}}$ between jets from the same and from different $\mathrm{W}$ bosons. The resulting systematic uncertainty for the $\mathrm{W}$ boson mass determination is $7 \mathrm{MeV}$ [86].

Bose-Einstein correlations. Due to Bose-Einstein statistics identical mesons from quark fragmentation tend to occupy the same place in momentum space. These effects are established in hadronic $\mathrm{Z}$ and $\mathrm{W}$ boson decays but they have not yet been confirmed between mesons stemming from different $\mathrm{W}$ bosons in $\mathrm{e}^{+} \mathrm{e}^{-} \rightarrow \mathrm{W}^{+} \mathrm{W}^{-}$. The uncertainty associated with imperfectly known correlations between mesons from different $\mathrm{W}$ bosons may lead to a bias in the determination of the $\mathrm{W}$ boson mass. A common systematic uncertainty of $35 \mathrm{MeV}$ [86] has been taken into account.

Combined results for W boson mass and width at LEP-II. The combination of all results for mass and total decay width of the W boson obtained by the four LEP experiments including also the threshold measurements [82,87-89] yields [90]

$$
\begin{gathered}
m_{\mathrm{W}}=80.376 \pm 0.033 \mathrm{GeV}, \\
\Gamma_{\mathrm{W}}=2.196 \pm 0.083 \mathrm{GeV} .
\end{gathered}
$$

The average is dominated by the $\mathrm{q} \overline{\mathrm{q}} \ell v_{\ell}$ channel because of the additional FSI errors in the $q \bar{q} q \bar{q}$ channel. The result is still preliminary.

\subsection{W boson measurements at the Tevatron}

At the Tevatron, $\mathrm{W}$ and $\mathrm{Z}$ bosons are produced in protonantiproton collisions: $\mathrm{p} \overline{\mathrm{p}} \rightarrow \mathrm{W}(\mathrm{Z})+\mathrm{X}$ where $\mathrm{X}$ denotes the residual hadronic system from the $\mathrm{p} \overline{\mathrm{p}}$ collision recoiling 
against the $\mathrm{W}(\mathrm{Z})$ boson. For the selection of $\mathrm{W}$ bosons only the leptonic decay modes $\mathrm{W} \rightarrow \mathrm{e}(\mu)+v_{\mathrm{e}}\left(v_{\mu}\right)$ are used, the purely hadronic mode suffers from too high background. The topology for a leptonic $\mathrm{W}$ boson decay is an isolated electron or muon together with the residual hadronic system from the $\mathrm{p} \overline{\mathrm{p}}$ collision. The neutrino is invisible but can give a sizable missing energy and momentum. Since remnant particles are lost along, or close to the beam pipe, the invariant mass of the $\mathrm{W}$ boson cannot be fully reconstructed. However, the plane transverse to the beam pipe is used to impose constraints on energy and momentum. The transverse part of the hadronic recoil opposite the $\mathrm{W}$ boson is measured. The transverse neutrino momentum is inferred to be opposite the vector sum of the lepton and hadronic recoil transverse momenta. The transverse mass, $m_{\text {trans }}$, is calculated as

$$
m_{\text {trans }}^{2}=2 p_{\mathrm{T}}^{\ell} p_{\mathrm{T}}^{\nu}\left(1-\cos \Phi_{\ell \nu}\right),
$$

where $\Phi_{\ell v}$ is the angle between the direction of the lepton momentum and the missing energy vector (neutrino) in the transverse plane. The transverse mass is smaller than the invariant mass of the decaying $\mathrm{W}$ boson and approaches it when the $\mathrm{W}$ boson decays in the transverse plane. This gives a cut-off which allows the determination of the $\mathrm{W}$ boson mass from the upper edge of the transverse mass distribution. The $\mathrm{W}$ boson mass is derived from fits to the measured transverse mass and momentum distributions. The absolute scale of the $\mathrm{W}$ boson mass is determined from leptonic $\mathrm{Z}$ boson decays, $\mathrm{Z} \rightarrow \mathrm{e}^{+} \mathrm{e}^{-}$ $\left(\mu^{+} \mu^{-}\right)$, and calibrating the measured $Z$ boson mass using the precise $\mathrm{Z}$ boson mass measurement at LEP. But the $\mathrm{Z}$ boson decay samples are limited and hence the determination of the absolute energy and momentum scale is the largest source of systematic errors for the $\mathrm{W}$ boson mass measurement.

The analyses of data collected during the Run-I phase (1988-1995) with the CDF and DØ detector are final for W boson mass measurement $[91,92]$ and for the $\mathrm{W}$ boson width measurement $[93,94]$ and were combined taking into account correlated systematic uncertainties [95]:

$$
\begin{aligned}
& m_{\mathrm{W}}=80.452 \pm 0.059 \mathrm{GeV} \\
& \Gamma_{\mathrm{W}}=2.102 \pm 0.106 \mathrm{GeV}
\end{aligned}
$$

The measurements for the Run-II phase started in 2001 and are still ongoing. The preliminary combined result for $\mathrm{W}$ mass measurements at the Tevatron is given in [96]:

$$
m_{\mathrm{W}}=80.420 \pm 0.031 \mathrm{GeV} \text {. }
$$

\subsection{World average of $W$ boson mass measurement}

The results on the mass and total width of the $\mathrm{W}$ boson obtained at LEP-II and the Tevatron are in good agreement; the combination of the results assuming no correlations yields the preliminary world averages of $\mathrm{W}$ boson mass and width [90, 96, 97, 99],

$$
\begin{aligned}
& m_{\mathrm{W}}=80.399 \pm 0.023 \mathrm{GeV} \\
& \Gamma_{\mathrm{W}}=2.085 \pm 0.042 \mathrm{GeV} .
\end{aligned}
$$

The left plot in figure 17 summarizes the results for the $\mathrm{W}$ boson mass measurements.
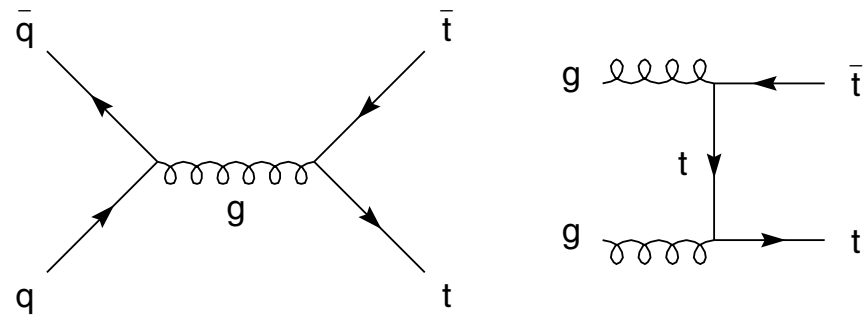

Figure 16. Generic Feynman diagrams of t $t \bar{t}$ production in $\mathrm{p} \overline{\mathrm{p}}$ collisions on parton level.

\subsection{Top quark production at the Tevatron}

In 1995, the top quark was discovered in proton-antiproton interactions recorded at the Tevatron collider by the experiments CDF [100] and DØ [101]. At this time, the electroweak measurements predicted $m_{\mathrm{t}}=173_{-13}^{+12} \mathrm{GeV}$ for $m_{\mathrm{H}}=300 \mathrm{GeV}$, with additional uncertainties of ${ }_{-20}^{+18} \mathrm{GeV}$ varying the Higgs boson mass between 60 and $1000 \mathrm{GeV}$. The Tevatron result, $m_{\mathrm{t}} \approx 175$, was in good agreement with this prediction. The precision on $m_{\mathrm{t}}$ has been substantially improved since that time.

In proton-antiproton collisions the top quarks are produced by the process $\mathrm{p} \overline{\mathrm{p}} \rightarrow \mathrm{t} \overline{\mathrm{t}}+X$; where $X$ denotes the $\mathrm{p} \overline{\mathrm{p}}$ remnant recoiling against the $\mathrm{t} \overline{\mathrm{t}}$ system. The lowest order Feynman diagrams on parton level contributing to top quark production are QCD mediated processes as shown in figure 16. Top quarks are also produced without their antiparticle via electroweak interactions but the cross section is smaller by a factor of two. Furthermore, it is much more easy to purify a sample of top quarks in $\mathrm{t} \overline{\mathrm{t}}$ events than in single top quark events. Hence the precision of the top quark mass measurement is higher if the $t \bar{t}$ process is used.

Because of its large mass the top quark decays faster than the hadronization to top-flavour bound states can happen. Since the Cabibbo-Kobayashi-Maskawa matrix element $V_{\mathrm{tb}}$ dominates, the top quark decays mainly to $\mathrm{Wb}$ states,

$$
\mathrm{t} \rightarrow \mathrm{bW}, \quad \mathrm{W} \rightarrow \mathrm{f} \overline{\mathrm{f}}^{\prime}\left(\mathrm{f}, \mathrm{f}^{\prime} \neq \mathrm{t}\right) .
$$

The classification of the $\mathrm{t} \overline{\mathrm{t}}$ pair events depends on the decay modes of the $\mathrm{W}$ boson-hadronic, semileptonic and leptonic.

In hadronic events (called the all jets channel), $\mathrm{t} \overline{\mathrm{t}} \rightarrow$ $\mathrm{b} \overline{\mathrm{b}} \mathrm{q} \overline{\mathrm{q}} \mathrm{q} \overline{\mathrm{q}}$, all $\mathrm{W}$ boson decay products are quarks. This gives a $t \bar{t}$ event signature with six jets or even more due to gluon radiation, together with the so-called 'underlying event'. The latter includes all particles from a single $\mathrm{p} \overline{\mathrm{p}}$ collision except the $t \bar{t}$ process. The kinematics of the top quarks can be reconstructed up to combinatorial ambiguities in associating light quark jets to the correct $\mathrm{W}$ boson and combining $\mathrm{W}$ bosons with b-quark jets to top quarks. The number of combinations is reduced using b-tagging to identify the two b-jets. But the QCD multi-jet background remains high and the resulting low efficiencies and purities limit the precision for the top mass measurement using this channel.

In principle, semileptonic events, $\mathrm{t} \overline{\mathrm{t}} \rightarrow \mathrm{b} \overline{\mathrm{b}} \mathrm{q} \overline{\mathrm{q}} \ell \bar{v}_{\ell}$ (called the lepton plus jets channel) allow a complete reconstruction of the invariant masses of the decaying top quarks. However, it is 


\section{W-Boson Mass $[\mathrm{GeV}]$}

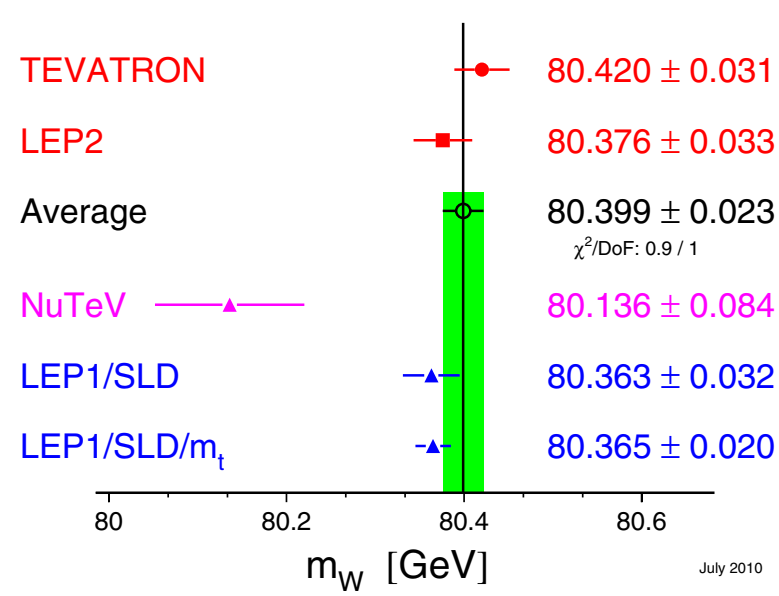

Top-Quark Mass $[\mathrm{GeV}]$

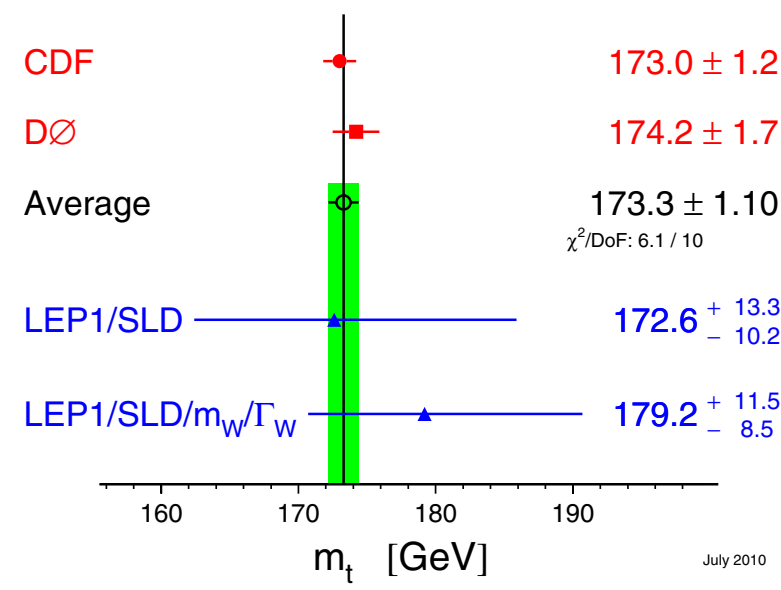

Figure 17. Left: results on the mass of the $\mathrm{W}$ boson, $m_{\mathrm{W}}$. The direct measurements of $m_{\mathrm{W}}$ at LEP-II (preliminary) and at Run-I of the Tevatron (top) are compared with the indirect determinations (bottom). The NuTeV result (see section 7.7) interpreted in terms of $m_{\mathrm{W}}$ is shown separately. Right: results on the mass of the top quark. The direct measurements of $m_{\mathrm{t}}$ at Run-I of the Tevatron (top) are compared with the indirect determinations (bottom). See also references $[96,98,103]$.

necessary to consider the complete production and decay chain including the hadronic system recoiling against the $\mathrm{t} \overline{\mathrm{t}}$ system. The identification of the b-quarks is important but also in this case combinatorial ambiguities remain. The advantage of this channel is the high branching fraction of about $30 \%$.

Top quark events with leptonic $\mathrm{W}$ boson decays, $\mathrm{t} \overline{\mathrm{t}} \rightarrow$ $\mathrm{b} \overline{\mathrm{b}} \ell \bar{v}_{\ell} \ell \bar{v}_{\ell}$, have final states with two b-jets, two high $p_{\mathrm{T}}$ leptons and missing transverse energy due to the two neutrinos. This dilepton channel suffers from a low branching fraction of about $5 \%$ but this is partially compensated by the low background.

Both, CDF and DØ measure the mass in both the dilepton and lepton plus jets channels; CDF also includes the measurement of all-jet events. The status of the top mass measurement is summarized in [103], the actual value of $m_{\mathrm{t}}$ from Tevatron is

$$
m_{\mathrm{t}}=173.32 \pm 0.56(\text { stat }) \pm 0.89(\text { syst }) \mathrm{GeV} .
$$

Assuming Gaussian systematic uncertainties and adding in quadrature yields a total uncertainty of $1.1 \mathrm{GeV}$. Due to the larger luminosity collected in Run-II as well as new mass analysis techniques, the mass of the top quark is now known with an accuracy of $0.75 \%$ which is a substantial improvement since the end of LEP operation. The precision is limited by systematic uncertainties dominated by the jet energy scale uncertainty and is expected to improve as larger data samples are collected. The top quark mass given in equation (134) is in good agreement with indirect measurements at the $\mathrm{Z}$ pole (see table 5) and is used as constraint to derive more precise information on the Higgs boson mass. The right plot in figure 17 summarizes the results for the top quark mass measurements. A detailed review of top physics at the Tevatron is given in [102].

\subsection{Constraints from measurements at low energies}

Parity violation in atoms. Parity violation in atoms (APV) arises from electroweak interactions between $\mathrm{S}$-shell electrons and quarks in nucleons. The chirality of the $\mathrm{Z}$ boson leads to the non-conservation of parity in highly forbidden transitions. The strength of this effect depends on the weak nuclear charge, $Q_{\text {W, }}$, which is the sum of the weak charges of all constituents of the atomic nucleus,

$$
Q_{\mathrm{W}}(Z, N)=-2\left[(2 Z+N) C_{1 \mathrm{u}}+(Z+2 N) C_{1 \mathrm{~d}}\right],
$$

with $N$ neutrons and $Z$ protons. The weak charges, $C_{1 \mathrm{q}}$, can be expressed in terms of effective vector and axial-vector coupling constants,

$$
C_{1 \mathrm{q}}=2 g_{\mathrm{Ae}} g_{\mathrm{Vq}} \text {. }
$$

The most precise measurements of $Q_{\mathrm{W}}$ have been obtained by measuring the polarization dependent rates of forbidden transitions in caesium [104, 105]. Over the last years a large amount of effort has been spent to evaluate the corrections and to control systematic effects. After corrections due to nuclear many-body perturbation theory and QED radiation, the weak charge has the value [106]

$$
Q_{\mathrm{W}}(\mathrm{Cs})=-72.65 \pm 0.28((\exp ) \pm 0.36 \text { (theor) }
$$

and agrees with the Standard model prediction $Q_{\mathrm{W}}(\mathrm{Cs})=$ $-73.19 \pm 0.13[106,107]$ within $1 \sigma$.

Parity violation in Moller scattering. A precision measurement of the effective weak neutral current coupling governing low energy Møller scattering, $\mathrm{e}^{-} \mathrm{e}^{-}$, has been performed by the E-158 collaboration at SLAC. A polarized electron beam with $E=48 \mathrm{GeV}$ scattering off unpolarized electrons in a liquid hydrogen target was used to measure the left-right asymmetry $A_{\mathrm{LR}}$ at $Q^{2}=0.026 \mathrm{GeV}^{2}$. The leftright asymmetry can be expressed in terms of the electroweak mixing angle resulting in [108-110]

$$
\sin ^{2} \theta_{\text {eff }}\left(Q^{2}\right)=0.2397 \pm 0.0013,
$$



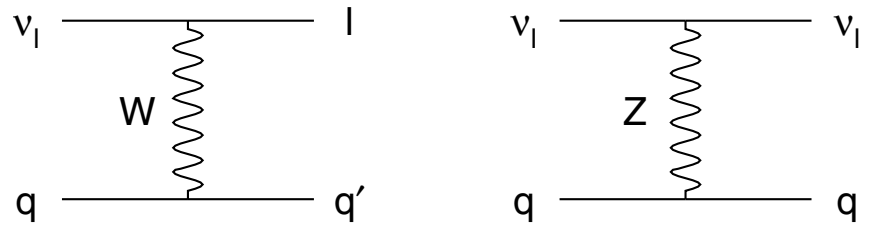

Figure 18. Feynman diagrams in neutrino-nucleon scattering on parton level. Left: charged current reaction. Right: neutral current reaction.

or, using the SM running of the electroweak mixing angle with $Q^{2}$,

$$
\sin ^{2} \theta_{\overline{\mathrm{MS}}}\left(m_{\mathrm{Z}}\right)\left(m_{\mathrm{Z}}\right)=0.2330 \pm 0.0015 .
$$

The effective electroweak mixing angle, $\sin ^{2} \theta_{\text {eff }}^{\text {lept }}$, is obtained by adding +0.00029 [9] to $\sin ^{2} \theta_{\overline{\mathrm{MS}}}\left(m_{\mathrm{Z}}\right)$.

Neutrino-nucleon scattering. Neutrino-nucleon scattering allows the measurement of both charged and neutral weak current interactions. The process is shown in lowest order in figure 18; neutrino or anti-neutrino interact via $\mathrm{W}$ or $\mathrm{Z}$ boson exchange in the $t$-channel with a quark in the nucleons of the target material. For the weak charged current, the right-handed component to the chiral quark couplings is zero, $g_{\mathrm{R}}=0$. But for the neutral currents the chiral quark couplings contain the term $Q \sin ^{2} \theta_{\mathrm{W}}$. By measuring the ratios of charged and neutral current processes the weak mixing angle can be determined at energies far below the $\mathrm{Z}$ resonance.

The ratio of neutral to charged current cross sections for either $v$ or $\bar{v}$ scattering from isoscalar targets of $\mathrm{u}$ - and d-quarks is

$$
R^{\nu(\bar{v})} \equiv \frac{\sigma((\bar{v}) N \rightarrow(\bar{v}) X)}{\sigma\left((\bar{v}) N \rightarrow \ell^{-(+)} X\right)}=g_{\mathrm{L}}^{2}+r^{-1} g_{\mathrm{R}}^{2},
$$

where

$$
r \equiv \frac{\sigma\left(\bar{v} N \rightarrow \ell^{+} X\right)}{\sigma\left(v N \rightarrow \ell^{-} X\right)} \approx \frac{1}{2},
$$

where $g_{\mathrm{L}, \mathrm{R}}^{2}$ is the quadratic sum of the left- and right-handed quark couplings. Corrections to $R^{v(\bar{v})}$ result from the presence of heavy sea quarks, the production of heavy quarks in the target, higher-order terms in the cross section and any isovector component in the light quarks in the target. Large uncertainties arise if a final state charm quark is produced from d- or s-quark in the nucleon. To reduce uncertainties from charm quark production the Paschos-Wolfenstein relation [111] is considered:

$$
\begin{aligned}
R_{-} & \equiv \frac{\sigma\left(v_{\mu} N \rightarrow v_{\mu} X\right)-\sigma\left(\bar{v} N \rightarrow \bar{v}_{\mu} X\right)}{\sigma\left(v_{\mu} N \rightarrow \mu^{-} X\right)-\sigma\left(\bar{v}_{\mu} N \rightarrow \mu^{+} X\right)}=\frac{R^{\nu}-r R^{\bar{\nu}}}{1-r} \\
& =g_{\mathrm{L}}^{2}-g_{\mathrm{R}}^{2}=\frac{1}{2}-\sin ^{2} \theta_{\mathrm{W}} .
\end{aligned}
$$

Up to small electroweak corrections $R_{-}$measures the on-shell electroweak mixing angle. The subscript $\mu$ indicates that a muon (anti-) neutrino beam has been used; the charged current reactions produce a primary muon in the final state while the neutral current reactions do not. The muon traverses the detector, the hadronic shower is confined to a small target volume. Thus, the discrimination between CC and NC reactions is defined by the extension of the event. It is more difficult to measure $R_{-}$than $R^{v}$ because the neutral current scattering processes of $v$ and $\bar{v}$ yield identical observed final states which can be only distinguished knowing the initial state neutrino.

The value for $R_{-}$was obtained from the distributions of measured CC and NC data, Monte Carlo simulations of the neutrino and anti-neutrino beam spectra, neutrino interactions in the target and the detector response; radiative corrections were included. Translated into the on-shell electroweak mixing angle, NuTeV published as final result [112]

$$
\begin{aligned}
\sin ^{2} \theta_{\mathrm{W}} \equiv & 1-m_{\mathrm{W}}^{2} / m_{\mathrm{Z}}^{2} \\
= & 0.22773 \pm 0.00135(\text { stat. }) \pm 0.00093 \text { (syst.) } \\
& -0.00022 \frac{m_{\mathrm{t}}^{2}-(175 \mathrm{GeV})^{2}}{(50 \mathrm{GeV})^{2}} \\
& +0.00032 \ln \left(m_{\mathrm{H}} / 150 \mathrm{GeV}\right)
\end{aligned}
$$

where $\rho=\rho_{\mathrm{SM}}$ is assumed. This result depends on $m_{\mathrm{t}}$ and $m_{\mathrm{H}}$ and is therefore sensitive to these masses. The value of the weak mixing angle implies

$$
m_{\mathrm{W}}=80.14 \pm 0.08 \mathrm{GeV} .
$$

From a simultaneous fit to $\sin ^{2} \theta_{\mathrm{W}}$ and the $\rho$ parameter the $\mathrm{NuTeV}$ collaboration obtained

$$
\rho=0.99789 \pm 0.00405, \quad \sin ^{2} \theta_{\mathrm{W}}=0.22647 \pm 0.00311
$$

with a correlation coefficient between $\rho$ and $\sin ^{2} \theta_{\mathrm{W}}$ of 0.85 [112]. The result can also be interpreted in terms of the isoscalar combinations of effective neutral current quark couplings, $\left(g_{\mathrm{L}, \mathrm{R}}^{\text {eff }}\right)^{2}=\left(u_{\mathrm{L}, \mathrm{R}}^{\text {eff }}\right)^{2}+\left(d_{\mathrm{L}, \mathrm{R}}^{\text {eff }}\right)^{2}$ at $\left\langle Q^{2}\right\rangle \approx 20 \mathrm{GeV}$ :

$$
\begin{aligned}
& \left(g_{\mathrm{L}}^{\text {eff }}\right)^{2}=0.30005 \pm 0.00137 \\
& \left(g_{\mathrm{L}}^{\text {eff }}\right)^{2}=0.03076 \pm 0.00110 .
\end{aligned}
$$

\subsection{Global electroweak fit}

The measurements and fit results are summarized in table 6 [97,98]. The measured $\mathrm{Z}$ pole observables are listed in the second column and are complemented by the results for the direct measurements of top quark mass and $\mathrm{W}$ boson mass and width. The quoted errors include the systematic uncertainties given in the third column. The input parameters to the global electroweak fit procedure are $\Delta \alpha_{\text {had }}^{(5)}\left(m_{\mathrm{Z}}^{2}\right), \alpha_{\mathrm{S}}$, $m_{\mathrm{Z}}, m_{\mathrm{t}}$ and $\log _{10}\left(m_{\mathrm{H}} / \mathrm{GeV}\right)$. An additional input parameter, not shown in the table, is the Fermi constant $G_{\mathrm{F}}$, determined from the $\mu$ lifetime, $G_{\mathrm{F}}=1.16637(1) \times 10^{-5} \mathrm{GeV}^{-2}$ [113]. The relative error of $G_{\mathrm{F}}$ is comparable to that of $m_{\mathrm{Z}}$; both errors have negligible effects on the results of the global fit. The global fit within the Standard Model has been performed successively combining the $\mathrm{Z}$ pole results and measurements of $m_{\mathrm{t}}, m_{\mathrm{W}}$ and $\Gamma_{\mathrm{W}}$; the individual steps of this procedure are compiled in table 7. The top mass is constrained by the result measured at the Tevatron. Derived 
Table 6. Summary of measurements included in the analyses of the five SM input parameters [97,98]. The top 15 results are included in the $\mathrm{Z}$ pole and the high- $Q^{2}$ fit, while the bottom three results are only used in the high- $Q^{2}$ fit. The total errors in column 2 include the systematic errors listed in column 3. The SM results in column 4 and the pulls in column 5 are derived from the SM analysis of all 18 results.

\begin{tabular}{lclcr}
\hline & $\begin{array}{l}\text { Measurement with } \\
\text { total error }\end{array}$ & $\begin{array}{l}\text { Systematic } \\
\text { error }\end{array}$ & $\begin{array}{l}\text { Standard } \\
\text { model fit }\end{array}$ & Pull \\
\hline$\Delta \alpha_{\text {had }}^{(5)}\left(m_{\mathrm{Z}}^{2}\right)[71]$ & $0.02758 \pm 0.00035$ & 0.00034 & 0.02768 & -0.3 \\
$m_{\mathrm{Z}}(\mathrm{GeV})$ & $91.1875 \pm 0.0021$ & $0.0017^{\mathrm{a}}$ & 91.1874 & 0.0 \\
$\Gamma_{\mathrm{Z}}(\mathrm{GeV})$ & $2.4952 \pm 0.0023$ & $0.0012^{\mathrm{a}}$ & 2.4959 & -0.3 \\
$\sigma_{\text {had }}^{0}(\mathrm{NB})$ & $41.540 \pm 0.037$ & $0.028^{\mathrm{a}}$ & 41.478 & 1.7 \\
$R_{\ell}^{0}$ & $20.767 \pm 0.025$ & $0.007^{\mathrm{a}}$ & 20.742 & 1.0 \\
$A_{\mathrm{FB}}^{0, \ell}$ & $0.0171 \pm 0.0010$ & $0.0003^{\mathrm{a}}$ & 0.01645 & 0.7 \\
$\mathcal{A}_{\ell}\left(P_{\tau}\right)$ & $0.1465 \pm 0.0032$ & 0.0016 & 0.1481 & -0.5 \\
$\mathcal{A}_{\ell}(\mathrm{SLD})$ & $0.1513 \pm 0.0021$ & 0.0011 & 0.1481 & 1.5 \\
$R_{\mathrm{b}}^{0}$ & $0.21629 \pm 0.00066$ & 0.00050 & 0.21579 & 0.8 \\
$R_{\mathrm{c}}^{0}$ & $0.1721 \pm 0.0030$ & 0.0019 & 0.1722 & 0.0 \\
$A_{\mathrm{FB}}^{0, \mathrm{~b}}$ & $0.0992 \pm 0.0016$ & 0.0007 & 0.1038 & -2.9 \\
$A_{\mathrm{FB}}^{0, \mathrm{c}}$ & $0.0707 \pm 0.0035$ & 0.0017 & 0.0742 & -1.0 \\
$\mathcal{A}_{\mathrm{b}}$ & $0.923 \pm 0.020$ & 0.013 & 0.9347 & -0.6 \\
$\mathcal{A}_{\mathrm{c}}$ & $0.670 \pm 0.027$ & 0.015 & 0.6683 & 0.1 \\
+ corr. matrix [86] & & & & \\
$\sin ^{2} \theta_{\mathrm{eff}}^{\text {lept }}\left(Q_{\mathrm{FB}}^{\text {had }}\right)$ & $0.2324 \pm 0.0012$ & 0.0010 & 0.23139 & 0.8 \\
$m_{\mathrm{t}}(\mathrm{GeV})$ & $173.3 \pm 1.1$ & 0.9 & 173.4 & -0.1 \\
$(\mathrm{Run}-\mathrm{I}+\mathrm{II}[103])$ & & & & \\
$(\mathrm{LEP}-\mathrm{II}+$ Tevatron $)$ & & & & \\
$m_{\mathrm{W}}(\mathrm{GeV})$ & $80.399 \pm 0.023$ & & 2.0920 & -0.2 \\
$\Gamma_{\mathrm{W}}(\mathrm{GeV})$ & $2.085 \pm 0.042$ & & & \\
\hline
\end{tabular}

${ }^{\mathrm{a}}$ Only common systematic errors are indicated.

Table 7. Results [97, 98] of the fits to (1) all $\mathrm{Z}$ pole data (LEP-I and SLD) plus direct $m_{\mathrm{t}}$ determination, (2) all $\mathrm{Z}$ pole data plus direct $m_{\mathrm{W}}$ and $\Gamma_{\mathrm{W}}$ determinations, (3) all $\mathrm{Z}$ pole data plus direct $m_{\mathrm{t}}, m_{\mathrm{W}}, \Gamma_{\mathrm{W}}$ determinations (i.e. all high- $Q^{2}$ results). As the sensitivity to $m_{\mathrm{H}}$ is logarithmic, both $m_{\mathrm{H}}$ as well as $\log \left(m_{\mathrm{H}} / \mathrm{GeV}\right)$ are quoted. The bottom part of the table lists derived results for $\sin ^{2} \theta_{\text {eff }}^{\text {lept }}, \sin ^{2} \theta_{\mathrm{W}}$ and $m_{\mathrm{W}}$.

\begin{tabular}{llll}
\hline & $\begin{array}{l}(1) \\
\text { all } \mathrm{Z} \text { pole data } \\
\text { plus } m_{\mathrm{t}}\end{array}$ & $\begin{array}{l}\text { (2) } \\
\text { all } \mathrm{Z} \text { pole data } \\
\text { plus } m_{\mathrm{W}}, \Gamma_{\mathrm{W}}\end{array}$ & $\begin{array}{l}\text { all } \mathrm{Z} \text { pole data } \\
\text { plus } m_{\mathrm{t}}, m_{\mathrm{W}}, \Gamma_{\mathrm{W}}\end{array}$ \\
\hline$m_{\mathrm{t}}(\mathrm{GeV})$ & $173.3 \pm 1.1$ & $179.2_{-8.5}^{+11.6}$ & $173.4 \pm 1.1$ \\
$m_{\mathrm{H}}(\mathrm{GeV})$ & $117_{-40}^{+58}$ & $146_{-81}^{+241}$ & $89_{-26}^{+35}$ \\
$\log \frac{m_{\mathrm{H}}}{\mathrm{GeV}}$ & $2.07 \pm 0.18$ & $2.16_{-0.35}^{+0.42}$ & $1.95_{-0.16}^{+0.15}$ \\
$\alpha_{\mathrm{S}}\left(m_{\mathrm{Z}}^{2}\right)$ & $0.1190 \pm 0.0027$ & $0.1190 \pm 0.0028$ & $0.1185 \pm 0.0026$ \\
$\chi^{2} /$ d.o.f. $(P)$ & $16.0 / 11(14 \%)$ & $16.8912(15 \%)$ & $17.3 / 13(19 \%)$ \\
$\sin ^{2} \theta_{\text {eff }}^{\text {lept }}$ & $0.23149 \pm 0.00016$ & $0.23143 \pm 0.00014$ & $0.23138 \pm 0.00013$ \\
$\sin ^{2} \theta_{\mathrm{W}}$ & $0.22328 \pm 0.00040$ & $0.22287 \pm 0.00036$ & $0.22301 \pm 0.00028$ \\
$m_{\mathrm{W}}(\mathrm{GeV})$ & $80.365 \pm 0.020$ & $80.386 \pm 0.018$ & $80.379 \pm 0.015$ \\
\hline
\end{tabular}

quantities such as the $\mathrm{W}$ boson mass and width or the effective weak mixing angle, correspondingly, are then constrained by independent measurements of these parameters given in sections 7.5 and 7.6 .

Based on the fit results including all constraints from high-energy measurements, Standard Model values for the observables are predicted. The central values are listed in the second-right column of table 6 . The values for the pull which describes the difference between measurement and fit results in units of the total measurement errors are given in the last row and visualized in figure 22 . The strong coupling constant derived in the global fit is in good agreement with the world average, $\alpha_{\mathrm{S}}=0.1176 \pm 0.0020$ [9].
The $\mathrm{W}$ boson mass is treated as derived quantity that depends on the top quark mass and the Higgs boson mass as described in section 2.3. The $\mathrm{Z}$ pole measurements constrain $m_{\mathrm{W}}$ and $m_{\mathrm{t}}$ as shown in figure 19 by the dashed line representing the $68 \%$ C.L. contour in the $\left(m_{\mathrm{t}}, m_{\mathrm{W}}\right)$ plane. These indirect measurements are in good agreement with the results from direct $m_{\mathrm{W}}$ measurements at LEPII/Tevatron and direct $m_{\mathrm{t}}$ measurements at the Tevatron (solid line contour in figure 19). The Standard Model band is given by the relationship for the masses and calculated for fixed $\Delta \alpha_{\text {had }}^{(5)}\left(m_{\mathrm{Z}}^{2}\right)$ assuming various Higgs boson masses. An additional uncertainty arises if the uncertainty of the vacuum polarization, $\Delta \alpha_{\text {had }}^{(5)}\left(m_{\mathrm{Z}}^{2}\right)=0.02758 \pm 0.00035$, is taken into 


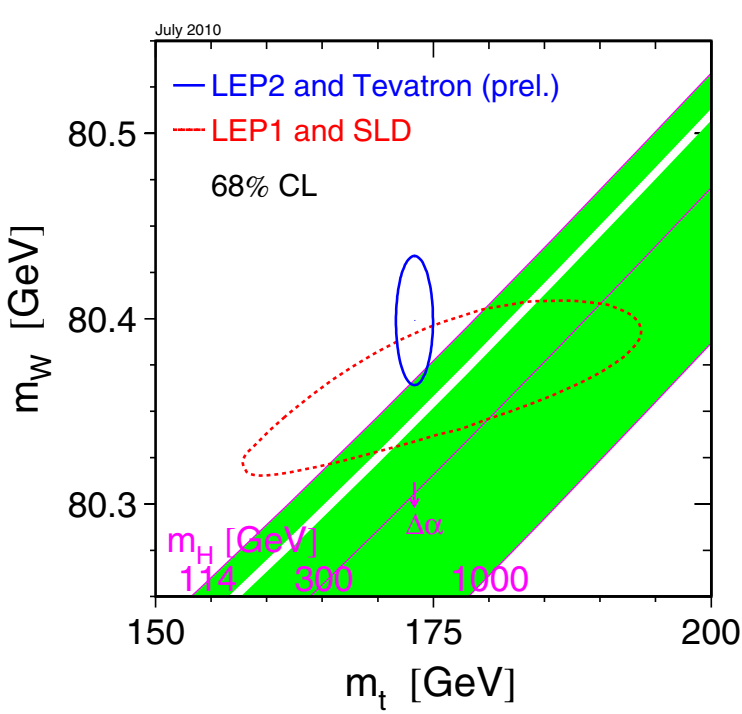

Figure 19. Contour curves of $68 \%$ probability in the $\left(m_{\mathrm{t}}, m_{\mathrm{W}}\right)$ plane [98]. The shaded band shows the SM prediction based on the value for $G_{\mathrm{F}}$ for various values of the Higgs boson mass and fixed $\Delta \alpha_{\text {had }}^{(5)}\left(m_{Z}^{2}\right)$; varying the hadronic vacuum polarization by

$\Delta \alpha_{\text {had }}^{(5)}\left(m_{\mathrm{Z}}^{2}\right)=0.02758 \pm 0.00035$ yields an additional uncertainty on the SM prediction shown by the arrow labelled $\Delta \alpha$.

account. The figure demonstrates that the combined data prefer a light Higgs boson. Figure 20 illustrates the interplay of the values fitted for $m_{\mathrm{t}}$ and $m_{\mathrm{W}}$ and their relation to the Higgs boson mass using the results of the direct $m_{\mathrm{W}}$ and $m_{\mathrm{t}}$ measurements as constraints. The plots visualize the comparison of the results for $m_{\mathrm{t}}, m_{\mathrm{W}}$ and $m_{\mathrm{H}}$ given in columns 3 and 4 of table 6 . On the left-hand plot of figure 20 the result for the top quark mass measurement at the Tevatron is checked against the indirect result from the fit to the $\mathrm{Z}$ pole data including also constraints from $\mathrm{W}$ boson mass and widths measurements. The righthand plot shows the comparison of the direct $\mathrm{W}$ boson mass measurements at LEP-II and the Tevatron with indirect LEPI/SLD measurements at the $\mathrm{Z}$ pole including also the top mass result from Tevatron. The indirect determination of the top quark mass is much less precise than that of the $\mathrm{W}$ boson mass. Therefore the constraint from direct measurements is essential to derive bounds on the Higgs boson mass.

To verify the consistency of the Standard Model measurements at low energies and at the $\mathrm{Z}$ pole, the values derived in the most significant fit (last column in table 7) have been used to predict the Standard Model couplings at low energies. The values for the parameters $Q_{W}, \sin ^{2} \theta_{\mathrm{W}}^{\text {on-shell }}$ and the couplings $g_{\nu \text { Lud }}^{2}, g_{\nu \text { Rud }}^{2}$ are compared with the results presented in section 7.7. The results as well as the pull of the measurements from the predictions of the best-fit model are listed in table 8, where the pull is defined as the absolute value of the difference between measurement and expectation in units of the measurement uncertainty. The agreement is good, only the $\mathrm{NuTeV}$ result for the values of the left-handed quark couplings deviates by 2.9 standard deviation from the Standard Model prediction. Possible large theoretical uncertainties in the area of radiative corrections and QCD effects influencing this measurement are discussed in [114-118]. Up to now a conclusive explanation of the disagreement has not been found.
Interpretations with new physics effects cannot account for the full effect because new phenomena are already constraint by other measurements.

\subsection{Global fit and the mass of the Higgs boson}

The Higgs boson mass is of special interest in the global fit. The $\chi^{2}$ curve, $\Delta \chi^{2}\left(m_{\mathrm{H}}\right)=\chi_{\min }^{2}\left(m_{\mathrm{H}}\right)-\chi_{\min }^{2}$, derived in a fit using all 18 results given in table 6 is shown in figure 21. The logarithmic mass scale corresponds to the logarithmic dependence of electroweak loop corrections on the Higgs boson mass. The associated band includes theoretical uncertainties in the Standard Model. The electroweak fits are performed using the hadronic contribution to the electromagnetic coupling constant, $\Delta \alpha_{\text {had }}^{(5)}\left(m_{\mathrm{Z}}^{2}\right)=0.02758 \pm$ 0.00035 . The $\Delta \chi^{2}$ curve is slightly changed if the fits are performed with $\Delta \alpha_{\text {had }}^{(5)}\left(m_{Z}^{2}\right)=0.02749 \pm 0.00012$ from [73].

Including all theoretical uncertainties, the one-sided $95 \%$ CL upper limit for $m_{\mathrm{H}}$ given with $\Delta \chi^{2}\left(m_{\mathrm{H}}\right)<2.7$ is [98]

$$
m_{\mathrm{H}}<158 \mathrm{GeV} \text {. }
$$

Measurements at LEP exclude $m_{\mathrm{H}}<114.4 \mathrm{GeV}$ [7]. If this limit is taken into account the upper Higgs boson mass limit

$$
m_{\mathrm{H}}<185 \mathrm{GeV}
$$

is obtained. Recent searches at the Tevatron exclude also the range $158 \mathrm{GeV}<m_{\mathrm{H}}<175 \mathrm{GeV}$ at the $95 \%$ C.L. [120]. The excluded regions for the Higgs boson mass from direct searches are also depicted in figures 20 and 21. The global fit does not include the results of electroweak measurements at low energies. They have only little influence on the fitted Higgs boson mass but the NuTeV measurement yields high $\chi^{2}$ values. A light Higgs boson is predicted by almost all individual analyses except the analysis of the forwardbackward asymmetry of b-jets which is compatible with a larger Higgs boson mass of few hundred gigaelectron volts (see also figure 12). This suggests that the Higgs boson or something equivalent should exist and will be detected at the LHC. The discovery of a Higgs boson signal different from these predictions would clearly indicate that the minimal Standard Model is not correct. But it could also be that there is no Higgs boson and another mechanism to assign masses to the particles is at work. However, without a Higgs boson the WW scattering process violates the perturbative unitarity unless new resonances or strong interactions appear. Indications of these phenomena will be seen at the LHC.

The status of the precision electroweak fits is shown in figure 22. All measured values for the observables are compared with the predictions using the results obtained in the global fit. The agreement is excellent, except the pole forward-backward asymmetry for b-quarks. However, the final evaluation of these discrepancies requires more precise measurements.

\subsection{Future precision measurements at the $Z$ pole}

It would be very attractive to perform again measurements at the $\mathrm{Z}$ boson resonance, with substantially higher precision and 

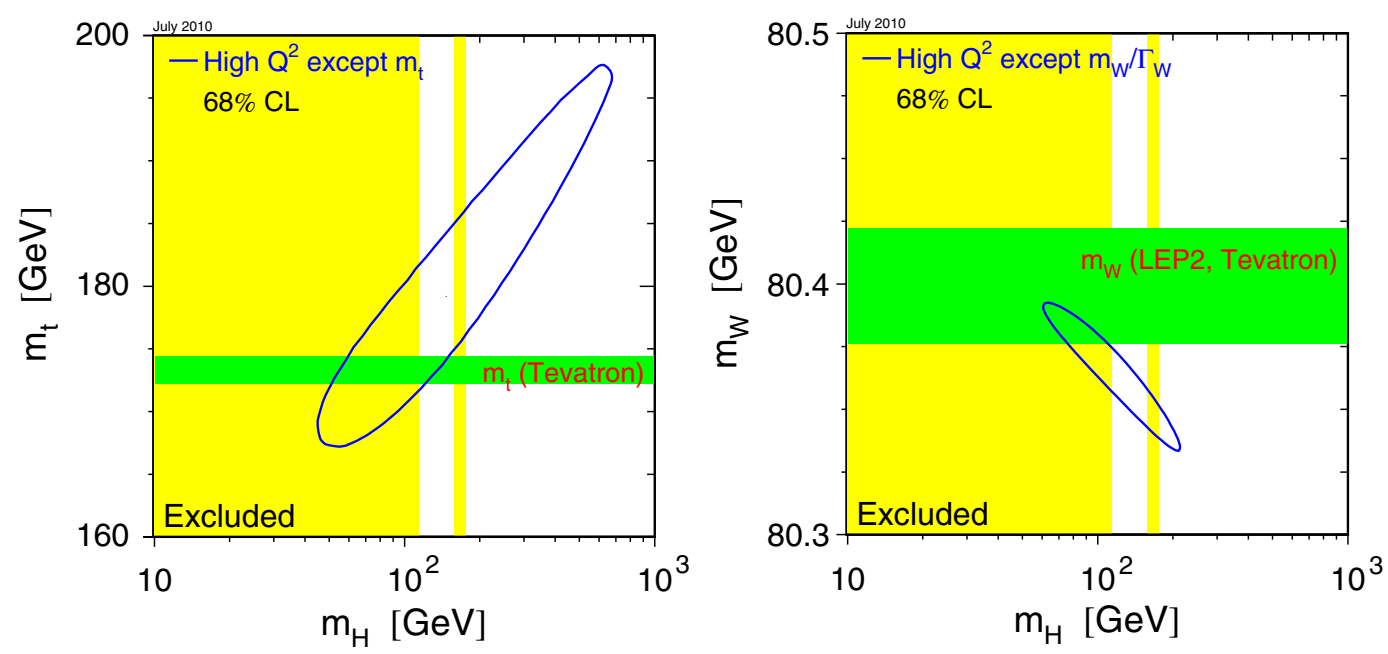

Figure 20. Dependence of Higgs boson mass on top quark and $\mathrm{W}$ boson mass determination [98]. Left: the 68\% confidence level contours in the $\left(m_{\mathrm{t}}, m_{\mathrm{H}}\right)$ plane for the fit to all data except the direct measurement of $m_{\mathrm{t}}$. The result from direct $m_{\mathrm{t}}$ measurement is indicated by the horizontal band of $\pm 1 \sigma$ width. Right: the $68 \%$ confidence level contours in the $\left(m_{\mathrm{W}}, m_{\mathrm{H}}\right)$ plane for the fit to all data except the direct measurement of $m_{\mathrm{W}}$. The result from direct $m_{\mathrm{W}}$ measurement is indicated by the horizontal band of $\pm 1 \sigma$ width. The vertical bands show the $95 \%$ CL exclusion limit on $m_{\mathrm{H}}$ from direct searches at LEP and Tevatron.

Table 8. Summary of predictions for results obtained in low- $Q^{2}$ processes derived from the fit to all high- $Q^{2}$ data $[97,98]$

\begin{tabular}{lccr}
\hline & $\begin{array}{l}\text { Measurement with } \\
\text { total error }\end{array}$ & $\begin{array}{l}\text { Standard model } \\
\text { high- } Q^{2} \text { fit }\end{array}$ & Pull \\
\hline APV [119] & & & \\
$Q_{\mathrm{W}}(\mathrm{Cs})$ & $-72.74 \pm 0.46$ & -72.920 & 0.4 \\
$\begin{array}{l}\mathrm{M} \varnothing 1 \text { ller [108-110] } \\
\sin ^{2} \theta_{\mathrm{MS}}\left(m_{\mathrm{Z}}\right)\end{array}$ & $0.2330 \pm 0.0015$ & 0.23115 & 1.2 \\
$\nu \mathrm{N}[112]$ & & & \\
$g_{v \text { Lud }}^{2}$ & $0.30005 \pm 0.00137$ & 0.30392 & -2.9 \\
$g_{v \text { Rud }}^{2}$ & $0.03076 \pm 0.00110$ & 0.03012 & 0.6 \\
\hline & & &
\end{tabular}

including the knowledge about top quark, W boson and Higgs boson parameters after years of LHC running. A modification of the Standard Model Higgs sector or any other kind of new physics influences directly the radiative corrections and becomes noticeable in precision observables as e.g. the weak mixing angle. With the GigaZ option at future $\mathrm{e}^{+} \mathrm{e}^{-}$ colliders like ILC or CLIC even more precise measurements at the $\mathrm{Z}$ pole would be possible. At the ILC [121] a luminosity corresponding to a billion $\mathrm{Z}$ boson decays collected within a few months of running will be delivered. This will allow measurements at the $\mathrm{Z}$ pole with extremely high precision, in particular the measurement of the $\mathrm{Zf} \overline{\mathrm{f}}$ coupling and the electroweak mixing angle with substantially improved accuracy. The most sensitive observable is the left-right asymmetry $A_{\mathrm{LR}}$. If the polarization of both beams, electrons and positrons, can be chosen independently, $A_{\mathrm{LR}}$ can be measured without the need for absolute polarimetry [121] yielding a statistical accuracy of about $\Delta A_{\mathrm{LR}}=3 \times 10^{-5}$. Conservatively, a final error of $\Delta \mathcal{A}_{\ell}=10^{-4}$ can be assumed corresponding to $\Delta \sin ^{2} \theta_{\text {eff }}^{\text {lept }}=1.3 \times 10^{-6}$. This is an improvement of more than one order of magnitude compared with the value obtained at LEP/SLD. To achieve this precision, one needs to know the fine structure constant at the scale $m_{\mathrm{Z}}$,

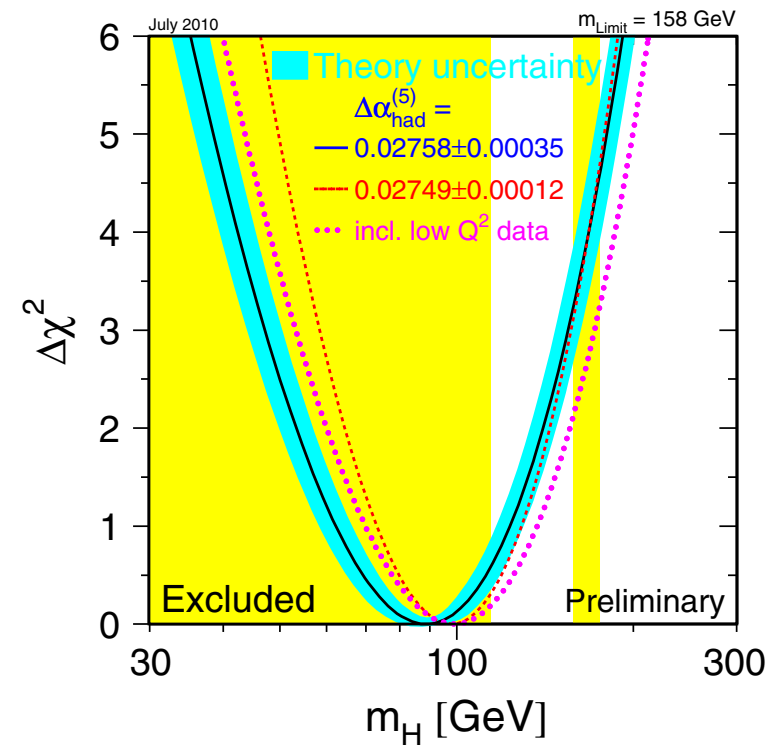

Figure 21. $\Delta \chi^{2}\left(m_{\mathrm{H}}\right)=\chi_{\min }^{2}\left(m_{\mathrm{H}}\right)-\chi_{\min }^{2}$ as a function of $m_{\mathrm{H}}$ [98]. The associated band represents the estimate of the theoretical uncertainty due to missing higher-order corrections. The broad vertical band shows the $95 \%$ confidence level exclusion limit on $m_{\mathrm{H}}$ derived from the direct search at LEP-II [7], the small vertical band shows direct search limits from Tevatron [120]. The dashed curve is the result obtained using the theory-driven determination $\Delta \alpha_{\text {had }}^{(5)}\left(m_{\mathrm{Z}}^{2}\right)=0.02749 \pm 0.00012$.

$\alpha\left(m_{\mathrm{Z}}^{2}\right)$, with a much higher precision than presently. The hadronic contribution, $\Delta \alpha_{\text {had }}^{(5)}\left(m_{Z}^{2}\right)$, can be determined with the necessary precision if the cross section $\mathrm{e}^{+} \mathrm{e}^{-} \rightarrow$ hadrons can be measured with an uncertainty of $1 \%$ roughly up to the $J / \Psi$ resonance [122].

The forward-backward asymmetry $A_{\mathrm{FB}}^{0, \mathrm{~b}}$ measured at LEP deviates from the Standard Model prediction by more than two standard deviations (see table 6). It could be a fluctuation or a sign of physics beyond the Standard Model. At a linear 


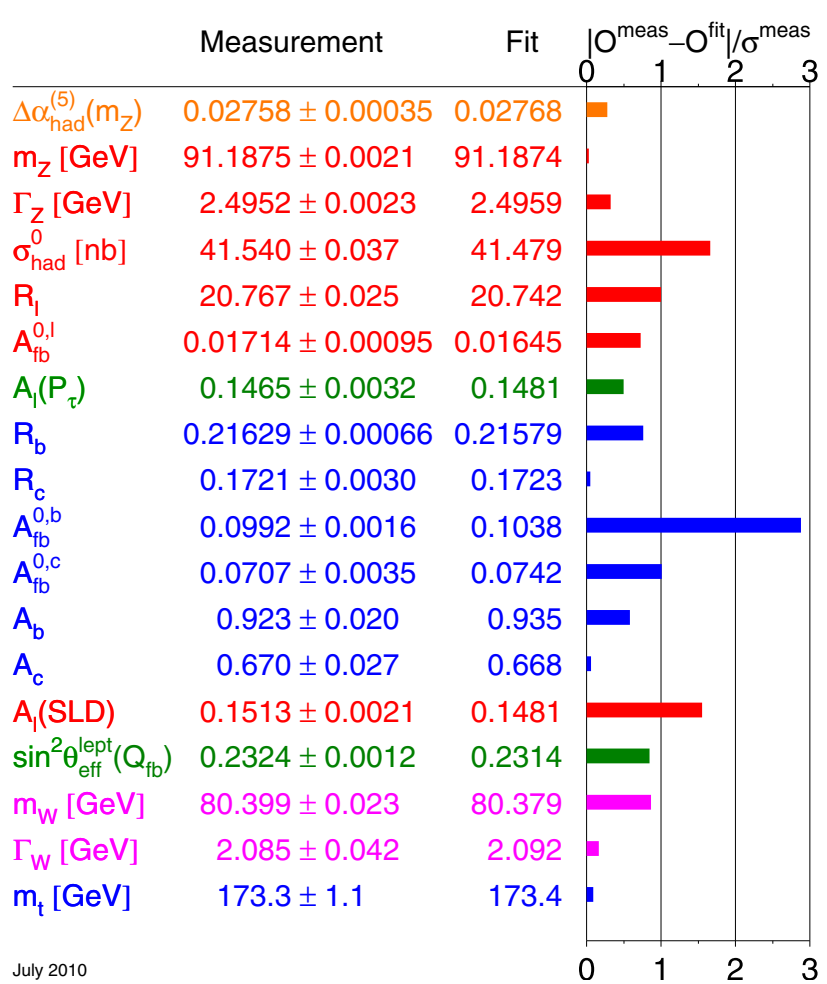

Figure 22. Comparison of measurements with expectation within the SM, calculated for the five SM input parameter values in the minimum of the global $\chi^{2}$ of the fit [98]. Also shown is the absolute value of pull of each measurement, where pull is defined as the absolute value of the difference of measurement and expectation in units of the measurement uncertainty.

collider with the GigaZ option, the asymmetry parameter $\mathcal{A}_{\mathrm{b}}=$ $2 g_{\mathrm{Vb}} g_{\mathrm{Ab}} /\left(g_{\mathrm{Vb}}^{2}+g_{\mathrm{Ab}}^{2}\right)$ can be measured one order of magnitude better than at LEP/SLD and without any dependence on the $\mathrm{Ze}^{+} \mathrm{e}^{-}$couplings. Similarly, the $R_{\mathrm{b}}$ measurement can be improved by a factor five. More details about the power of the GigaZ option can be found in $[121,123]$ and references therein.

\subsection{Parametrization of radiative corrections}

A commonly used parametrization of electroweak corrections which is convenient to indicate physics beyond the Standard Model is based on the $S T U$ parameters [124], extended by the parameter $\gamma_{b}$ for the b-quark sector [125]. The $T$ parameter is proportional to the difference between $\mathrm{W}$ and $\mathrm{Z}$ boson self-energies at $Q^{2}=0$, while $S(S+U)$ is associated with the difference between the $\mathrm{Z}(\mathrm{W})$ boson self-energy at $Q^{2}=m_{\mathrm{Z}}^{2}\left(m_{\mathrm{W}}^{2}\right)$ and $Q^{2}=0$. The $S T U$ parameters are related to the $\epsilon$ parameters [126] by

$$
\begin{gathered}
S \simeq+\epsilon_{3} \frac{4 \sin ^{2} \theta_{0}}{\alpha\left(m_{\mathrm{Z}}^{2}\right)}-c_{S}, \\
T \simeq \epsilon_{1} \frac{1}{\alpha\left(m_{\mathrm{Z}}^{2}\right)}-c_{T}, \\
U \simeq-\epsilon_{2} \frac{4 \sin ^{2} \theta_{0}}{\alpha\left(m_{\mathrm{Z}}^{2}\right)}-c_{U}, \\
\gamma_{\mathrm{b}} \simeq 2.29 \epsilon_{\mathrm{b}}-c_{\gamma} .
\end{gathered}
$$

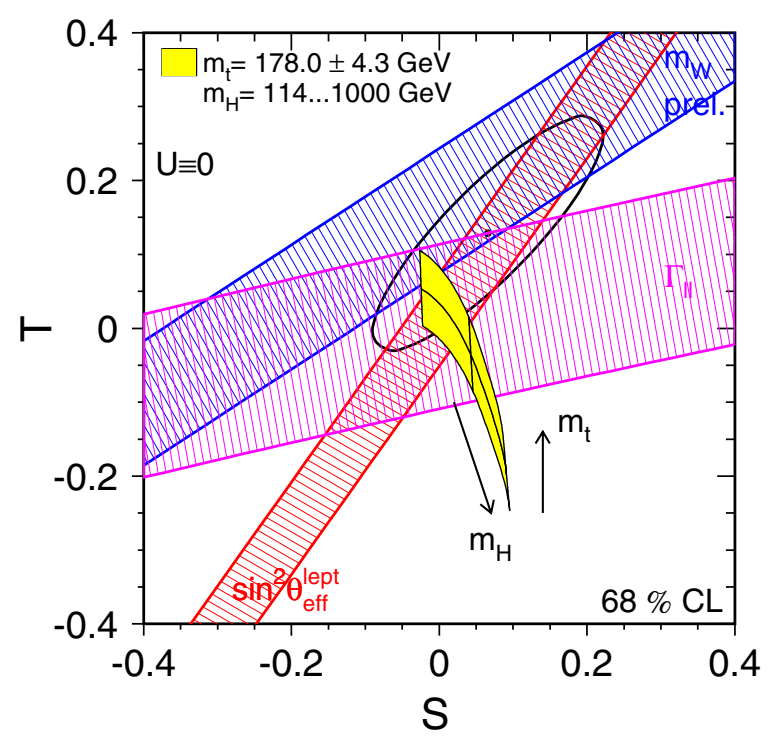

Figure 23. Contour curve of $68 \%$ probability in the $(T, S)$ plane, $U=0$ is imposed [32]. Also shown are \pm 1 standard deviation bands corresponding to the measurements of $\Gamma_{\ell \ell}, m_{\mathrm{W}}$ and $\sin ^{2} \theta_{\mathrm{eff}}^{\text {lept }}$. The shaded region shows the predictions within the SM for $m_{\mathrm{t}}=178.0 \pm 4.3 \mathrm{GeV}$ (Tevatron Run-I) and $m_{\mathrm{H}}=300_{-186}^{+700} \mathrm{GeV}$, for a fixed hadronic vacuum polarization of $\Delta \alpha_{\text {had }}^{(5)}\left(m_{\mathrm{Z}}^{2}\right)=0.02758$. The SM reference point at which all $S T U$ parameters vanish is chosen to be: $\Delta \alpha_{\text {had }}^{(5)}\left(m_{\mathrm{Z}}^{2}\right)=0.02758, \alpha_{\mathrm{S}}\left(m_{\mathrm{Z}}^{2}\right)=0.118, m_{\mathrm{Z}}=91.1875 \mathrm{GeV}$, $m_{\mathrm{t}}=175 \mathrm{GeV}, m_{\mathrm{H}}=150 \mathrm{GeV}$. The constraint $U=0$ is always applied. The direct measurement of $m_{\mathrm{W}}$ used here is preliminary. (Reprinted with permission from [32]. Copyright 2006 Elsevier.)

In the literature, the $S T U$ parameters are defined as shifts relative to a fixed set of $\mathrm{SM}$ values $c_{i}, i=S, T, U, \gamma$, so that $S=T=U=\gamma_{\mathrm{b}}=0$ at that point. Hence, the $S T U$ parameters measure deviations from the electroweak radiative corrections expected in the Standard Model. The $\epsilon$ parameters are defined such that they vanish in the approximation when only pure QED and QCD effects are taken into account,

$\epsilon_{1}=\Delta \rho$,

$\epsilon_{2}=\cos ^{2} \theta_{0} \Delta \rho+\frac{\sin ^{2} \theta_{0}}{\cos ^{2} \theta_{0}-\sin ^{2} \theta_{0}} \Delta r_{\mathrm{w}}-2 \sin ^{2} \theta_{0} \Delta \kappa^{\prime}$,

$\epsilon_{3}=\cos ^{2} \theta_{0} \Delta \rho+\left(\cos ^{2} \theta_{0}-\sin ^{2} \theta_{0}\right) \Delta \kappa^{\prime}$,

$\epsilon_{\mathrm{b}}=\frac{1}{2} \Delta \rho_{\mathrm{b}}$.

Corresponding to equation (35), the effective electroweak mixing angle, $\sin ^{2} \theta_{\text {eff }}^{\text {lept }}$ is related to $\sin ^{2} \theta_{0}$ given in equation (119) by

$$
\sin ^{2} \theta_{\mathrm{eff}}^{\text {lept }}==\kappa \sin ^{2} \theta_{0}=\left(1+\Delta \kappa^{\prime}\right) \sin ^{2} \theta_{0} .
$$

The $\mathrm{Z}$ pole measurements performed at LEP-I and SLD constrain the $\epsilon$ and $S T U$ parameters. The parameter $U$ contributes mainly to $m_{\mathrm{W}}$ and $\Gamma_{\mathrm{W}}$, and the value for $U$ predicted by the Standard Model is very small. Hence the constraint $U=0$ is imposed for the analysis. The fit result for the $S T U$ parameters is shown in figure 23, the values for $S T U$ are consistent with zero, indicating that there are no large unexpected electroweak radiative corrections. 

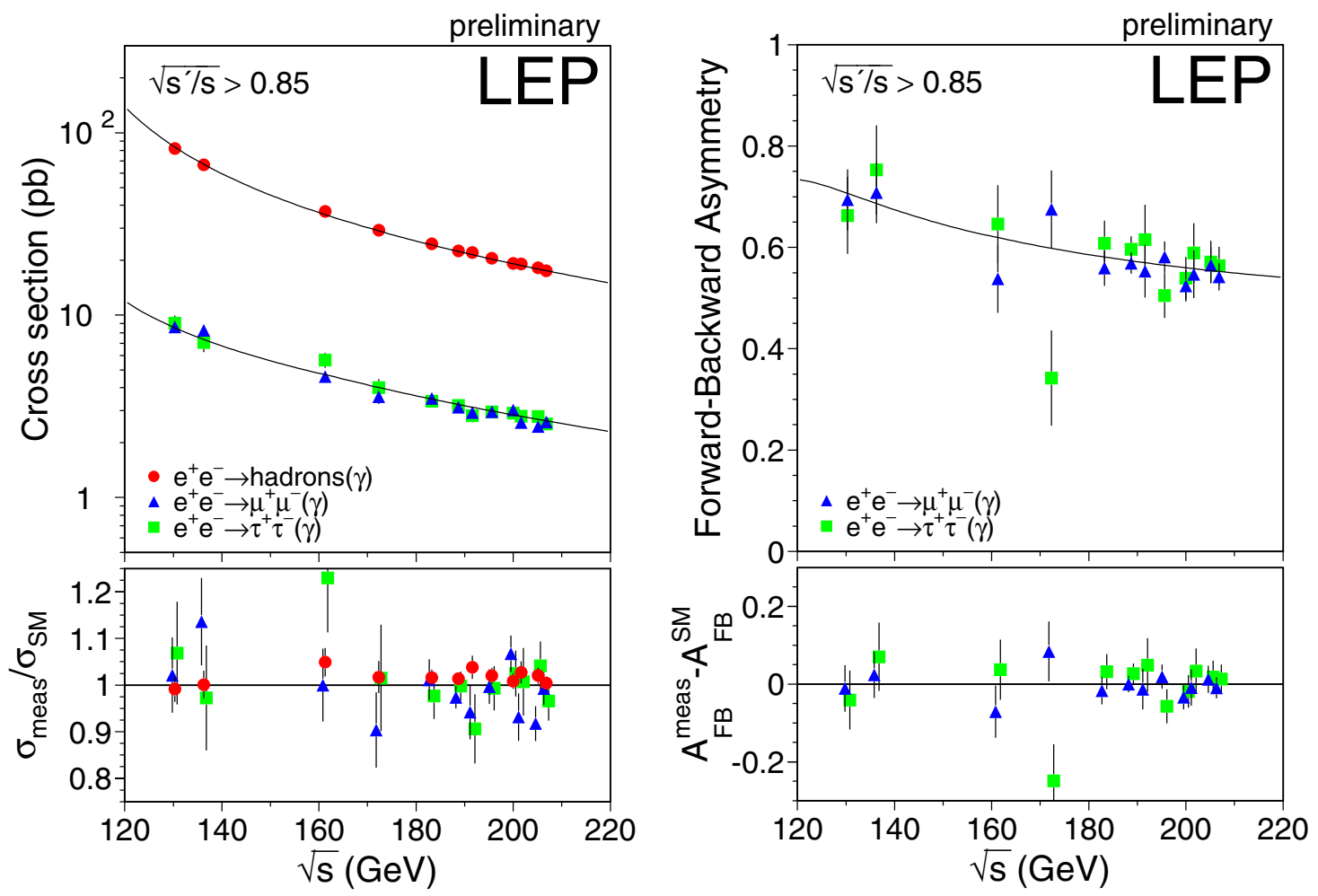

Figure 24. The cross section and forward-backward asymmetries for fermion-pair production measured at LEP-II at energies above the $\mathrm{Z}$ boson resonance, compared with the Standard Model expectations [86]. The measurements include the high-energy events, $\sqrt{s^{\prime}}>0.85$. The lower plots show the deviations of the measurements from the SM expectations.

\section{8. $\mathrm{e}^{+} \mathrm{e}^{-}$annihilation at energies above the $\mathrm{Z}$ boson}

From 1995 to 2000, at LEP-II an integrated luminosity of more than $700 \mathrm{pb}^{-1}$ per experiment was collected at energies between 130 and $208 \mathrm{GeV}$, substantially more than the initially expected luminosity of $500 \mathrm{pb}^{-1}$ per experiment. Although delivering high luminosity, the total number of fermion pairs is orders of magnitude below that produced on the $\mathrm{Z}$ boson resonance. Hence the statistical errors of the cross section and asymmetry measurements are at the level of a few per cent.

\subsection{Fermion-pair production above the $Z$ pole}

The selection of fermion-pair events at LEP-II energies is similar to that at LEP-I. One important feature arises from initial state radiation: the energy of the $\mathrm{e}^{+} \mathrm{e}^{-}$system is effectively reduced by photons emitted from the initial state electrons, (see figure 4), the so-called 'radiative return' to the $\mathrm{Z}$ boson resonance. To probe the Standard Model at high energies, the pure high-energy data are needed corresponding to events with high reduced centre-of-mass energies,

$$
\sqrt{s^{\prime}}=\sqrt{\left(1-E_{\gamma} / E_{\text {beam }}\right) s}>0.85 \sqrt{s} .
$$

Since most of the initial state photons disappear in the beam pipe, the reconstruction of $\sqrt{s^{\prime}}$ is performed using the invariant mass of the final state products.

Cross sections and asymmetries. At energies above the $\mathrm{Z}$ boson resonance none of the cross section contributions, $\sigma_{\gamma}, \sigma_{\gamma \mathrm{Z}}, \sigma_{\mathrm{Z}}$, dominates, all are numerically important. It is impossible to find expressions for the observables that are similarly convenient as the pseudo-observables used in the $\mathrm{Z}$ pole combinations. The LEP experiments measured the high-energy cross sections and asymmetries by selecting events with $\sqrt{s^{\prime}}>0.85$ [67]. Due to different experimental conditions as detector fiducial volume and experimental cuts, the measurements of each experiment were first corrected to a common signal definition before combining the results. The cross sections for the $\mathrm{e}^{+} \mathrm{e}^{-}, \mu^{+} \mu^{-}, \tau^{+} \tau^{-}$, and the inclusive hadronic final states as well as the forward-backward asymmetries of $\mu^{+} \mu^{-}$and $\tau^{+} \tau^{-}$final states have been averaged [86]. The current results for fermion-pair production in the range $130-208 \mathrm{GeV}$ are shown in figure 24 . The measurements agree well with the Standard Model predictions. Also the heavy flavour final states $b \bar{b}$ and $c \bar{c}$ at high energies were measured by the LEP experiments [127-133]. The selection procedures are similar to that at the $\mathrm{Z}$ pole. However, the ratio $R_{\mathrm{q}}$ represents the ratio of cross sections instead of $\mathrm{Z}$ boson partial widths, $R_{\mathrm{q}}=\sigma_{\mathrm{q} \overline{\mathrm{q}}} / \sigma_{\text {had }}$. The preliminary result of the combined heavy flavour measurements at LEP-II can be found in [86]. The measurements agree with the Standard Model predictions.

\subsection{Interpretation of LEP-II fermion-pair production measurements}

Direct searches for several new particles like supersymmetric particles, additional neutral gauge bosons, leptoquarks and extra dimensions have been performed using the LEP-II 
data. None of these new physics phenomena was observed. That implies that they do not exist or the masses of new particles are too high to be produced at energies up to $208 \mathrm{GeV}$. In the latter case there is chance to obtain new physics indirectly as deviation of measurements from the Standard Model predictions. New physics models yield additional contributions to the Lagrangian resulting in modified predictions of physics observables. The comparison of measurements and predictions based on these models excludes these scenarios or constrains their parameter range. Contact interactions are a general approach to describe new physics scenarios.

Contact interactions. In the low-energy limit, new interactions beyond the Standard Model can be parametrized in terms of effective four-fermion contact interactions [134, 135], similar as it was done by introducing weak interactions at low momentum transfer. The effective Lagrangian is

$$
\mathcal{L}_{\mathrm{CI}}=\sum_{i, j=\mathrm{L}, \mathrm{R}} \eta_{i j} \frac{g_{\mathrm{CI}}^{2}}{\Lambda^{2}}\left(\overline{\mathrm{e}}_{i} \gamma_{\mu} \mathrm{e}_{i}\right)\left(\bar{f}_{j} \gamma^{\mu} f_{j}\right)
$$

with the helicity coefficients $\eta_{i j}$ and the couplings $g_{\mathrm{CI}}$ (by convention $g_{\text {CI }}^{2}=4 \pi$ ). The parameter $\Lambda$ can be interpreted as compositeness scale, or it approximates effects of new particles, $\Lambda \approx m_{\mathrm{X}} / g_{\mathrm{X}}$. Contact interactions are assumed to preserve the chiral symmetry and the Standard Model matrix element is extended by an additional contact term. The new interactions could arise in fermion-pair production processes at lepton colliders or in hadron-hadron collisions producing lepton or quark pairs at the LHC by means of the Drell-Yan mechanism. The contact interactions modify the SM helicity amplitudes, so the amplitudes for the annihilation channel (equation (48)) are

$$
A_{i j}=Q_{\mathrm{f}} Q_{\mathrm{e}}+\frac{g_{i}^{\mathrm{e}} g_{k}^{\mathrm{f}}}{\cos \theta_{\mathrm{W}} \sin \theta_{\mathrm{W}}} \frac{s}{s-m_{\mathrm{Z}}^{2}+\mathrm{i} m_{\mathrm{Z}} \Gamma_{\mathrm{Z}}}+\frac{\eta_{i j} s}{\alpha \Lambda^{2}} .
$$

The parameter $\Lambda$ is determined by comparing the measurements with the predictions from contact interaction models given by the choice of $\eta_{i j}$. The LEP experiments have analysed the high-energy data and searched for deviations from the Standard Model based on the results derived in the electroweak global fits. No hint of new physics has been found and limits have been set for the scale of contact interactions. Depending on the coefficient $\eta_{i j}$ being \pm 1 or 0 , several models are chosen to obtain a sensitivity to new physics expressed as lower limit on $\Lambda$ for the corresponding model. The models studied are listed in table 9. Figure 25 summarizes the lower limits on $\Lambda$ for leptons and quarks. Universal couplings for leptons and universal couplings for up- and down-type quarks are assumed. Limits on $\Lambda$ derived for the individual flavours can be found in [86]. These results compare and exceed results obtained with HERA [136] and Tevatron [137, 138] data. For details on indirect searches for new physics phenomena see [86] and references therein.

The bounds on the contact terms $\Lambda$ can be interpreted in the framework of special models. A famous example is the
Table 9. Models of contact interaction.

\begin{tabular}{lrrrr}
\hline Model & $\eta_{L L}$ & $\eta_{R R}$ & $\eta_{L R}$ & $\eta_{R L}$ \\
\hline $\mathrm{LL}^{ \pm}$ & \pm 1 & 0 & 0 & 0 \\
$\mathrm{RR}^{ \pm}$ & 0 & \pm 1 & 0 & 0 \\
$\mathrm{VV}^{ \pm}$ & \pm 1 & \pm 1 & \pm 1 & \pm 1 \\
$\mathrm{AA}^{ \pm}$ & \pm 1 & \pm 1 & $\mp 1$ & $\mp 1$ \\
$\mathrm{LR}^{ \pm}$ & 0 & 0 & \pm 1 & 0 \\
$\mathrm{RL}^{ \pm}$ & 0 & 0 & 0 & \pm 1 \\
$\mathrm{~V}^{ \pm}$ & \pm 1 & \pm 1 & 0 & 0 \\
$\mathrm{~A}^{ \pm}$ & 0 & 0 & \pm 1 & \pm 1 \\
\hline
\end{tabular}

search for additional neutral gauge bosons which are predicted by many new physics models. It will be discussed in the next section which gives an overview of phenomena beyond the Standard Model.

\section{Beyond the Standard Model}

Although the Standard Model is in very good agreement with the measurements, there is no doubt that it is only an approximate effective theory. The Standard Model does not provide a unified description of all fundamental forces; gravity is not included. When trying to extend the validity of the Standard Model towards the scale of gravity, two severe problems arise: (1) the running of the weak, strong and electromagnetic gauge couplings with the energy points towards the unification of strong and electroweak forces in one single interaction at $M_{\mathrm{GUT}} \sim 10^{15}-10^{16} \mathrm{GeV}$. However, at the scale $M_{\mathrm{GUT}}$, these coupling constants are not fully identical as expected if the Standard Model should be embedded in the broader framework of a grand unified theory (GUT). (2) The so-called naturalness problem which is connected to the Higgs boson mass: quantum corrections due to loop insertions shift the Higgs boson mass, the most significant contribution comes from diagrams involving top quarks, $\Delta m_{\mathrm{H}}^{2} \sim m_{\mathrm{t}}^{2} \Lambda^{2}$. The loop corrections are divergent if $\Lambda$, the upper limit of the momentum in the loop integration, is sent to infinity. In order to keep the size of the mass shift under control, $\Lambda$ must either be limited by a cutoff at high energy or short distance, or contributions from new physics beyond the Standard Model cancel the divergence. In the absence of new physics, the Standard Model would be valid up to the GUT scale or even the Planck scale, and $\Lambda$ becomes about $10^{14}-10^{17}$ orders of magnitude larger than the electroweak scale, $v \approx 246 \mathrm{GeV}$. The precision electroweak measurements predict a light Higgs boson, $\mathcal{O}(100 \mathrm{GeV})$, but this can only be obtained if the bare Higgs boson mass is of the order of the GUT or Planck scale. A fine-tuning to the level $\left(v / M_{\mathrm{GUT}}\right)^{2} \approx 6 \times 10^{-28}$ would be necessary to remove the divergences in the radiative corrections by very exact cancellations. Such a procedure appears unnatural.

There is a large variety of new physics scenarios beyond the Standard Model which provide a solution to the two mentioned problems. They involve new dynamics of electroweak symmetry breaking and/or new concepts of space-time.

Since the naturalness problem is connected with the scale of electroweak symmetry breaking, the new ingredients are 

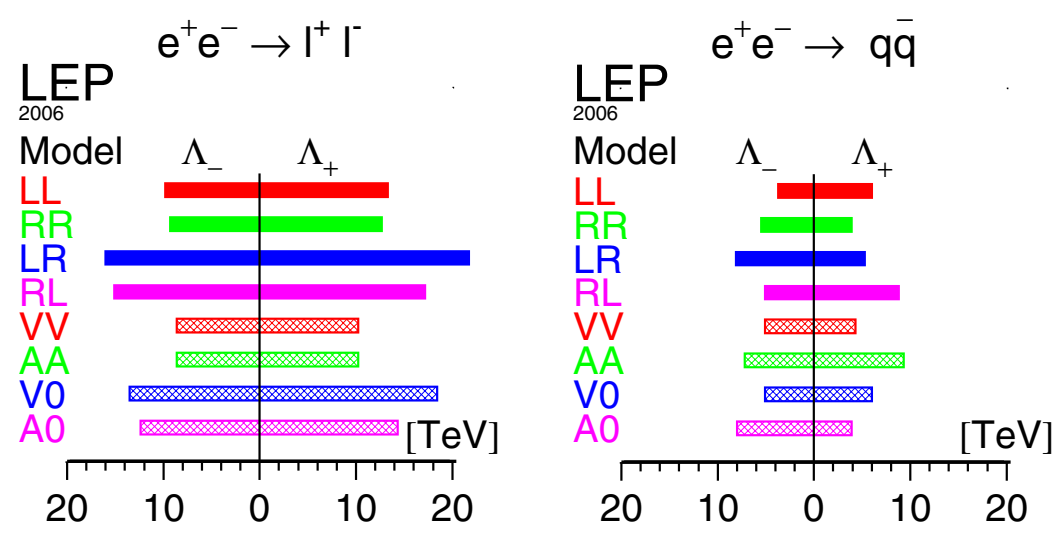

Figure 25. Lower limits on the scale $\Lambda$ of contact interactions at the 95\% C.L.; the superscripts + and - correspond to the models given in table 9. Left: limits derived from $\mu^{+} \mu^{-}$and $\tau^{+} \tau^{-}$final states combined assuming universal contact coupling to leptons. Right: limits derived from hadronic final states, $q \bar{q}$, assuming universal couplings for down-type and for up-type quarks. See also [86].

closely related to the physics of the Higgs sector and involve new particles and/or new interactions at the Terascale. New signals are expected which can be detected by direct signals or observing deviations from the Standard Model predictions. Furthermore, the new models need to address also the question of dark matter.

Among the plethora of scenarios, some examples are briefly presented. A review of new physics scenarios with focus on precision measurements at future linear $\mathrm{e}^{+} \mathrm{e}^{-}$colliders is given in [139].

\subsection{Supersymmetry}

Supersymmetry (SUSY) [140] is one of the theoretically best motivated extensions of the Standard Model. It is a symmetry relating fermions to bosons and is implemented by associating a new particle (called a sparticle) differing by one-half unit of spin with every known particle. The couplings of the new particles are related to those of the known particles. This leads to predictions for the production and decay rates of sparticles in terms of the unknown sparticle masses and other parameters. SUSY solves the two problems at the GUT scale at once: supersymmetric particle loops cancel exactly the quadratic divergences of Standard Model particle loops and the light Higgs boson mass as predicted by electroweak measurements is natural also for large scales. In addition, supersymmetric particle loops contribute to the running of the gauge couplings to allow their unification at the GUT scale. And there is another important point: SUSY seems to fit well to the results measured for the electroweak observables, in some cases even slightly better than the Standard Model. The electroweak observables are sensitive to virtual effects of supersymmetric particles. In [141, 142] the authors present the predictions of the electroweak observables in the Minimal Supersymmetric Standard Model (MSSM) taking into account quantum corrections. The results for $m_{\mathrm{t}}$ and $m_{\mathrm{W}}$ shown in figure 19 are compared with the predictions of both, SM and MSSM in figure 26. A comprehensive report on supersymmetric models and its comparison with experimental results can be found in [143].

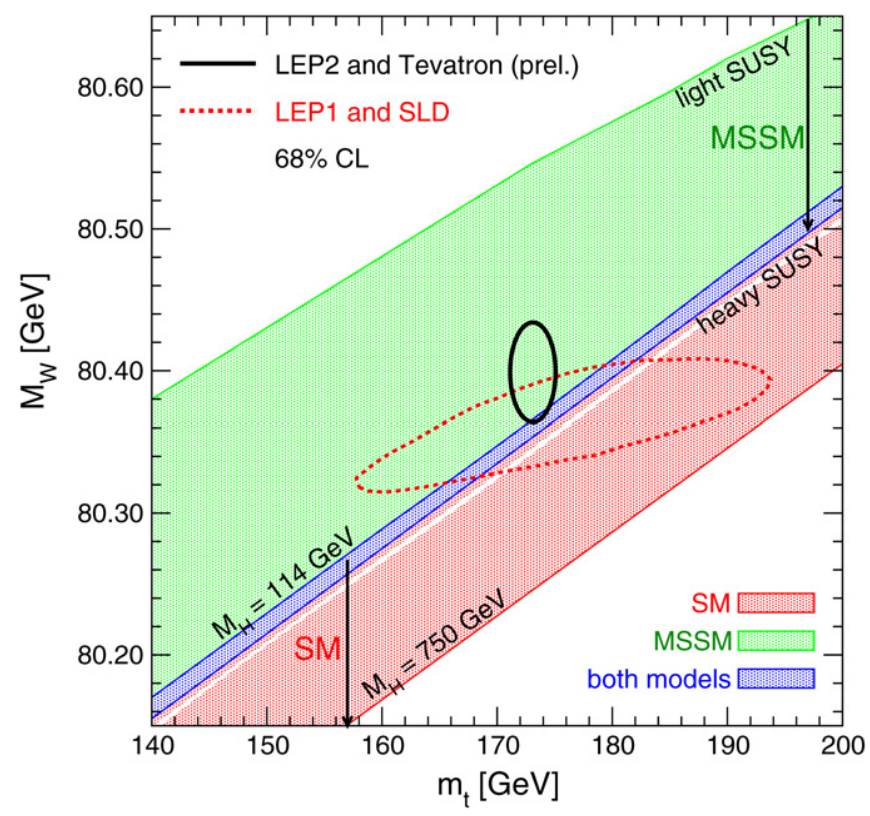

Figure 26. Contour curves of $68 \%$ probability in the $\left(m_{\mathrm{t}}, m_{\mathrm{W}}\right)$ plane compared with the predictions from the SM and the MSSM (see also reference [141]. The red (medium gray) and the blue (dark) shaded areas show the SM prediction, the green (light gray) and blue (dark) shaded regions show the predictions from the MSSM.

\subsection{Models with extra dimensions}

An interesting new direction is taken by models with extra spatial dimensions. The extra dimensions are compactified to a radius $R$ and in some models only gravitons propagate to the extra-dimensional space. $R$ is small enough to be consistent with current gravitational tests. The four-dimensional Planck mass, which is high because gravity is weak, $G_{N} \sim 1 / M_{\text {Planck }}^{(4)}$, is a fictitious mass scale and the fundamental gravity mass scale in the higher dimension can be close to the TeV scale. In a compactified extra dimension of radius $R$, the energy of a standing wave would be $E=n / R$ with $n$ as integer. Interpreting the standing wave as particle, a discrete mass spectrum, $m_{n}^{2}=n^{2} / R^{2}$, is expected, the so-called KaluzaKlein $(\mathrm{KK})$ tower. These towers of $\mathrm{KK}$ recurrences are the specific signature of models with extra dimensions. 
Models with large extra dimensions. In so-called large extra dimension models [144-147], like ADD, some of the compactified extra dimensions are sufficiently large and the Standard Model fields are confined to a four-dimensional brane immersed in a $d$-dimensional bulk; gravity feels the whole geometry and propagates in the bulk. The characteristic signal in collider experiments is the emission of KK gravitons obtained in event topologies with missing energy. Searches for graviton emission have been performed at LEP and at the Tevatron, no signals have been observed. The non-observation is interpreted in terms of lower limits on the fundamental $n$-dimensional Planck scale, $M_{\mathrm{D}}$, for a large extra dimensions scenario: The LEP data exclude values up to $M_{\mathrm{D}}=1.50 \mathrm{TeV}$ $(1.14,0.91,0.76 \mathrm{TeV})$ for $2(3,4,5)$ extra dimension(s) at the $95 \%$ C.L. [148]; measurements at the Tevatron yield $M_{\mathrm{D}}>1.40(1.15,1.04,0.98) \mathrm{TeV}$ for $n=2(3,4,5)$ extra dimensions [149].

The exchange of KK gravitons contributes also to fermionpair production [150-152]. Modelling the graviton exchange in fermion-pair production as contact interaction, the resulting angular distributions are quartic in the scattering angle, $\cos \theta$. The $s$-channel helicity amplitudes (equation (48)) are modified,

$$
\begin{aligned}
& A_{\mathrm{LL}, \mathrm{RR}}=A_{\mathrm{LL}, \mathrm{RR}}^{\mathrm{SM}}-\frac{\lambda \cdot s^{2}}{4 \pi \alpha M_{\mathrm{S}}^{4}}(2 \cos \theta-1) \\
& A_{\mathrm{LR}, \mathrm{RL}}=A_{\mathrm{LR}, \mathrm{RL}}^{\mathrm{SM}}-\frac{\lambda \cdot s^{2}}{4 \pi \alpha M_{\mathrm{S}}^{4}}(2 \cos \theta+1)
\end{aligned}
$$

where $M_{\mathrm{S}}$ is the ultraviolet cutoff scale of the sum over KK states in virtual graviton exchange ${ }^{1}$, and $|\lambda|$ is of the order 1. Similarly, also the SM $t$-channel amplitudes for the Bhabha scattering are changed. The virtual exchange of spin-2 gravitons yields a contribution to $A_{i j}$ that depends on the scattering angle $\cos \theta$. This is a specific feature of these models, hence the measurement of angular distributions of cross sections and asymmetries in fermion-pair production is very important to reveal the existence of large extra dimensions.

The combined LEP-II data have been used to search for indirect signals from large extra dimensions. The analysis of differential Bhabha cross sections yields lower bounds on $M_{\mathrm{S}}$,

$$
\begin{array}{ll}
M_{\mathrm{S}}>1.20 \mathrm{TeV} & \text { for } \lambda=+1, \\
M_{\mathrm{S}}>1.09 \mathrm{TeV} & \text { for } \lambda=-1 .
\end{array}
$$

at $95 \%$ C.L. [86].

The $\mathrm{D} \varnothing$ collaboration has analysed the di-electron and di-photon channels as well as the di-jet angular distributions to search for extra spatial dimensions. The measured distributions constrain the lower limits on $M_{\mathrm{S}}$ to values between 2.1 and $1.3 \mathrm{TeV}$ for 2 to 7 extra dimensions at 95\% C.L. [153].

\footnotetext{
$1 M_{\mathrm{D}}$ and $M_{\mathrm{S}}$ are of the same order of magnitude but the exact relation between them depends on the exact quantum gravity scenario. The conventions used in writing the effective Lagrangian can be obtained from [146, 150,151].
}

Warped extra dimension models. In the RS model [154], the geometry is not factorized and the metric in our four dimensions depends on the coordinate in the extra dimension ('warp' factor). Two four-dimensional branes are placed at different points in the fifth dimensional direction, and the space-time between two branes is part of a five-dimensional anti-de Sitter space. In this case, all masses in the fourdimensional space are exponentially scaled down with respect to the masses in five dimensions, $m_{5} \sim 1 / M_{\text {Planck. The }}$ weakness of gravitation is explained by the suppression of the graviton wave function at the SM brane. The KK modes of the graviton, however, can couple to the SM particles, and could be visible as spin-two resonances at colliders. The effects may also appear indirectly in production processes of Standard Model particles. The CDF collaboration performed a search for high-mass neutral resonances using di-muon data. No significant excess above the SM expectation has been observed, and the mass of the RS graviton is bound to $100 \mathrm{GeV}<M_{\mathrm{G}^{\star}}<921 \mathrm{GeV}$ at the $95 \%$ C.L. for $k^{2} / M_{\text {Planck }}=0.1\left(k^{2}\right.$ is the space-time curvature in the extra dimension) [155].

Universal extra dimension models. In models with universal extra dimensions (UED) [156], all Standard Model particles are assumed to propagate in a flat extra-dimensional space. With a suitable orbifold compactification, a phenomenologically viable model can be constructed. UED models are similar to a bosonic supersymmetric theory since the first KK modes play the role of superpartners in SUSY models but with different spins of the heavy excited particles. By introducing a KK parity, the lightest particle of the first KK excitations becomes stable and a potential dark matter candidate.

\subsection{Strong electroweak symmetry breaking}

In the Standard Model as well as in its supersymmetric extensions, the Higgs field is introduced as a fundamental degree of freedom. However, if new strong interactions exist, the symmetry breaking works also without Higgs bosons. The spontaneous breaking of global symmetries generates a set of massless Goldstone bosons. Longitudinal degrees of freedom and masses of gauge bosons are created by absorbing these Goldstone bosons. Several scenarios have been developed $[157,158]$ as an alternative to the standard Higgs mechanism. Recently, little Higgs models are discussed. They represent an 'intermediate option' between strong and weak symmetry breaking and account for a light Higgs boson mass as indicated by precision electroweak measurements.

Little Higgs models. If the Standard Model is viewed as an effective theory, new physics well above the weak scale can be parametrized in terms of higher-dimensional operators which are suppressed by inverse powers of the cutoff $\Lambda$. Precision measurements indicate a small Higgs boson mass $\mathcal{O}(100 \mathrm{GeV})$ and significant deviations have not been obtained. This translates into a cutoff $\Lambda \sim 10 \mathrm{TeV}$, considerably higher than the weak scale, $v$. The Higgs boson mass is quadratically 
sensitive to the cutoff scale via quantum corrections, so only a fine-tuning at the per cent level is necessary.

In little Higgs models (LHM) [159], the Higgs boson is a pseudo-Goldstone boson of a global symmetry spontaneously broken at a scale $f$. If $f \sim 1 \mathrm{TeV}$, the Higgs boson can be light, $m_{\mathrm{H}} \sim f / 4 \pi \approx 100 \mathrm{GeV}$. The LHM can be considered as an effective theory up to the cutoff $\Lambda \sim 4 \pi f \approx 10 \mathrm{TeV}$. The quadratic divergence of radiative corrections to $m_{\mathrm{H}}$ is cancelled at the one-loop level by extra gauge bosons and top partners.

In the minimal version of LHM, the 'Littlest Higgs Model', a symmetry pattern $\mathrm{SU}(5) \rightarrow \mathrm{SO}(5)$ is imposed. At the same time the gauge group $[\mathrm{SU}(2) \times \mathrm{U}(1)]^{2}$ is broken to its subgroup $\mathrm{SU}(2) \times \mathrm{U}(1)$, identified as SM gauge group. This leads to four additional massive vector bosons: an $\mathrm{SU}(2)$ triplet, $\mathrm{Z}_{\mathrm{H}}$, $\mathrm{W}_{\mathrm{H}}^{ \pm}$and a $\mathrm{U}(1)$ boson $\mathrm{A}_{\mathrm{H}}$. The masses of these bosons are of order $f$. An interesting class of little Higgs models are those with $\mathrm{T}$ parity [160] in which the new particles can be much lighter than $1 \mathrm{TeV}$ without conflict with the precision electroweak data; the lightest T-odd particle, a heavy photon, can be even lighter than a few hundred gigaelectron volt and provides a possible candidate for cold dark matter.

\subsection{New gauge bosons}

New gauge bosons in the intermediate $\mathrm{TeV}$ scale are motivated also by other theoretical approaches [161]. For instance, the breaking of grand unifying theories (GUTs) based on $\mathrm{SO}(10)$ or $\mathrm{E}_{6}$ symmetries, may leave one or several $\mathrm{U}(1)$ remnants unbroken down to $\mathrm{TeV}$ energies, before the symmetry reduces to the Standard Model symmetry. In the case of the $\mathrm{E}_{6}$ model, one has the possible breaking pattern

$$
\begin{aligned}
\mathrm{E}_{6} & \rightarrow \mathrm{SO}(10) \times \mathrm{U}(1)_{\psi} \rightarrow \mathrm{SU}(5) \times \mathrm{U}(1)_{\chi} \times \mathrm{U}(1)_{\psi} \\
& \rightarrow \mathrm{SM} \times \mathrm{U}(1)^{\prime},
\end{aligned}
$$

and the new $Z^{\prime}$ boson corresponding to the final $U(1)^{\prime}$ remnant, is a linear combination of the gauge bosons of the $\mathrm{U}(1)^{\prime}$ s generated in the two-step symmetry breaking, $\mathrm{Z}^{\prime}=$ $\mathrm{Z}_{\chi}^{\prime} \cos \beta+\mathrm{Z}_{\psi}^{\prime} \sin \beta$. The value $\beta=\arctan (-\sqrt{5 / 3})$ would correspond to a $\mathrm{Z}_{\eta}^{\prime}$ originating from the direct breaking of $\mathrm{E}_{6}$ to a rank-5 group in superstring inspired models. Another interesting option are left-right (LR) models, based on the group $\mathrm{SU}(2)_{\mathrm{R}} \times \mathrm{SU}(2)_{\mathrm{L}} \times \mathrm{U}(1)_{\mathrm{B}-\mathrm{L}}$ in which the new $\mathrm{Z}_{\mathrm{LR}}^{\prime}$ will couple to a linear combination of the right-handed and B-L currents with a parameter $\alpha_{\mathrm{LR}}^{2} \sim 3 g_{\mathrm{R}}^{2} / g_{\mathrm{L}}^{2}-1$. $\mathrm{Z}^{\prime}$ bosons modify the helicity amplitudes (equation (48)),

$$
\begin{aligned}
A_{i k}= & Q_{\mathrm{f}} Q_{\mathrm{e}}+\frac{g_{i}^{\mathrm{e}} g_{k}^{\mathrm{f}}}{\cos \theta_{\mathrm{W}} \sin \theta_{\mathrm{W}}} \frac{s}{s-m_{\mathrm{Z}}^{2}+\mathrm{i} m_{\mathrm{Z}} \Gamma_{\mathrm{Z}}} \\
& +\frac{g_{i}^{\mathrm{Z}^{\prime}, \mathrm{f}} g_{k}^{\mathrm{Z}^{\prime}, \mathrm{f}}}{\cos \theta_{\mathrm{W}}} \frac{s}{s-m_{\mathrm{Z}^{\prime}}^{2}+\mathrm{i} m_{\mathrm{Z}^{\prime}} \Gamma_{\mathrm{Z}^{\prime}}}
\end{aligned}
$$

with $i, k=\mathrm{L}, \mathrm{R}$ and $g_{\mathrm{Af}}^{\mathrm{Z}^{\prime}}=g_{\mathrm{L}}^{\mathrm{Z}^{\prime}, \mathrm{f}}-g_{\mathrm{R}}^{\mathrm{Z}^{\prime}, \mathrm{f}}$ and $g_{\mathrm{Vf}}^{\mathrm{Z}^{\prime}}=g_{\mathrm{L}}^{\mathrm{Z}^{\prime}, \mathrm{f}}+g_{\mathrm{R}}^{\mathrm{Z}^{\prime}, \mathrm{f}}$. Below the resonance, new gauge bosons appear as deviations from the Standard Model predictions due to $\gamma-\mathrm{Z}^{\prime}$ and $\mathrm{Z}-\mathrm{Z}^{\prime}$ interference terms. LEP data have been used to search for $Z^{\prime}$ bosons $[67,86,162]$. As already shown for the case of contact interactions, no significant deviation from the SM expectation has been obtained and lower limits on the mass of these hypothetical particles have been determined. Depending on the $\mathrm{Z}^{\prime}$ model, it has been found at the $95 \%$ C.L. that a $Z^{\prime}$ boson with couplings from $\mathrm{E}_{6}$ inspired models should have a mass $m_{\mathrm{Z}}^{\prime}>$ $430 \ldots 670 \mathrm{GeV}$, a $Z^{\prime}$ boson in left-right symmetric models yields $m_{Z^{\prime}}>800 \mathrm{GeV}$. A $Z^{\prime}$ boson with the same couplings as the Standard Model $\mathrm{Z}$ boson is excluded for masses below $1.9 \mathrm{TeV}$. These indirect bounds have to be compared with direct search limits from hadron colliders: the Tevatron experiments have not found any signal of extra neutral gauge bosons and exclude them up to $0.7-1.03 \mathrm{TeV}[155,163]$ for the models introduced above. $\mathrm{Z}^{\prime}$ bosons with masses up to about 4-5 TeV would be found at the $\operatorname{LHC}[164,165]$. Also future $\mathrm{e}^{+} \mathrm{e}^{-}$ colliders will extend the search reach substantially. Even if such a collider is operated below the production threshold of the new particles, measurements at high energies allow the detection of deviations from Standard Model predictions. The indirect sensitivity to $\mathrm{Z}^{\prime}$ bosons is determined by the precision of the measurements and scales with luminosity and energy,

$$
m_{\mathrm{Z}^{\prime}} \sim \mathcal{L}^{1 / 4} \cdot \sqrt{E_{\mathrm{cm}}}
$$

for $m_{\mathrm{Z}^{\prime}}>E_{\mathrm{cm}}$. Assuming a linear $\mathrm{e}^{+} \mathrm{e}^{-}$collider operating at $1 \mathrm{TeV}$, and a luminosity yielding the number of events as with LEP-II, the sensitivity will improve by a factor five and by yet another factor of three assuming this number of events for CLIC $(3 \mathrm{TeV})$. If a Z' boson would be found at the LHC, precise measurements at a linear collider allow the determination of the $\mathrm{Z}^{\prime}$ boson couplings and to conclude the underlying symmetry. But even if the LHC does not see an existing $Z^{\prime}$ boson, the sensitivity reach of $\mathrm{e}^{+} \mathrm{e}^{-}$colliders can suffice to detect the new particle. The $Z^{\prime}$ boson contributions depend on couplings normalized to the $\mathrm{Z}^{\prime}$ boson mass,

$$
g_{\mathrm{Af}}^{N}=g_{\mathrm{Af}}^{\mathrm{Z}^{\prime}} \sqrt{\frac{s}{m_{\mathrm{Z}^{\prime}}^{2}-s}} \quad g_{\mathrm{Af}}^{N}=g_{\mathrm{Vf}}^{\mathrm{Z}^{\prime}} \sqrt{\frac{s}{m_{\mathrm{Z}^{\prime}}^{2}-s}},
$$

and measurements of $g_{\mathrm{Af}}^{N} \neq 0$ and/or $g_{\mathrm{Vf}}^{N} \neq 0$ indicate new phenomena and discriminate between models without knowing the mass of the $\mathrm{Z}^{\prime}$ boson. Already with measurements at a $1 \mathrm{TeV} \mathrm{e}^{+} \mathrm{e}^{-}$collider the search reach of the LHC can be exceeded in the case of models with not too weak coupling [166]. The interplay between measurements at lepton and hadron colliders for future discoveries has been discussed over many years and the comparison between a future $\mathrm{e}^{+} \mathrm{e}^{-}$linear collider and the LHC is summarized in [167].

Of course, the Standard Model $\mathrm{Z}$ boson and the $\mathrm{Z}^{\prime}$ boson can mix, and the mixing modifies the electroweak parameters. Hence, the precision measurements performed at the $\mathrm{Z}$ boson resonance are especially sensitive to the $Z-Z^{\prime}$ mixing. The measurements at LEP/SLD are consistent with zero mixing (see for example [162]). It should be noted that $\rho=1$ at tree-level is assumed in most of the present analyses and studies concerning new gauge bosons. Models with $\rho \neq 1$ require new loop calculations leading to largely modified electroweak corrections and their relations to the Standard Model parameters. In [168] this is demonstrated in detail for the $\Delta r\left(m_{\mathrm{t}}\right)$ behaviour considering $\mathrm{Z}^{\prime}$ bosons in a specific leftright model without the restriction $\rho=1$. Accuracies expected 
for the GigaZ option of a future linear collider (see section 7.10) will help to elucidate the origin of potential new gauge bosons and the symmetry breaking mechanism responsible for the mixing. Even in the 'zero coupling' limit there could remain an observable contribution to the $\rho$ parameter.

\section{Summary and outlook}

The results obtained at LEP and SLD, complemented by recent measurements at the Tevatron, are a milestone in the history of precision electroweak measurements and form the present picture of the Standard Model. They have confirmed the Standard Model up to the level of quantum corrections. The properties of the $\mathrm{Z}$ boson are determined with an accuracy that will remain unchallenged for many years. In particular, the $\mathrm{Z}$ boson mass is now one of the most precisely measured quantities and its uncertainty will not be improved in future experiments.

One of the great triumphs is the measurement of the top quark mass: by analysing precision data at the $\mathrm{Z}$ pole, the mass was predicted years before the discovery of the top quark at the Tevatron experiments CDF and DØ in 1995. The result of the direct top mass measurement agrees well with the prediction from indirect measurements. Similarly, the mass of the W boson derived from precision measurements agrees with the direct measurements at LEP-II and at the Tevatron. Does this also repeat for the Higgs boson? The global analysis of precision data taken at the $\mathrm{Z}$ pole including the direct measurements of $m_{\mathrm{t}}$ and $m_{\mathrm{W}}$ yields a clear indication for a light Higgs boson. But so far the Higgs boson has not been found. Its discovery is expected at the LHC. The electroweak part of the Standard Model cannot be considered complete without establishing the Higgs sector by experiment. It is not sufficient to find the Higgs boson in the predicted mass range; its couplings and self-couplings must agree with that defined by the spontaneous symmetry breaking mechanism in the Standard Model.

New signals beyond the Standard Model have not been observed. Some observables differ from the Standard Model expectations by 2-3 standard deviations. Future experiments at the LHC and later on at a high-energy $\mathrm{e}^{+} \mathrm{e}^{-}$linear collider will elucidate whether this is due to statistical fluctuations or a first sign of new physics.

The LHC will extend the energy frontier beyond the electroweak scale and new phenomena are expected to become visible. One may ask whether precision measurements become less important in view of new discoveries at energies of few teraelectron volts. But it is clear that the LHC results alone cannot reveal all secrets of the nature of new particles. To understand the underlying theory that describes particles and their interactions - including phenomena such as dark matter-the consistency of LHC results has to be proven by complementing them with precision measurements at $\mathrm{e}^{+} \mathrm{e}^{-}$ colliders at very high energies, $\sqrt{s}>0.2 \mathrm{TeV}$. Furthermore, it would be very attractive to perform again measurements at the $\mathrm{Z}$ boson resonance with substantially higher precision including the knowledge about top quark, W boson and Higgs boson parameters after years of LHC running. With future precision measurements at $\mathrm{e}^{+} \mathrm{e}^{-}$colliders like ILC or CLIC this will be possible and offers a real global test of the Standard Model and physics beyond.

\section{Acknowledgments}

I would like to thank the members of the LEP, SLD and Tevatron electroweak working groups, in particular Martin Grünewald, for their work. It is a great pleasure to thank Peter Zerwas for encouragement, the careful reading of the manuscript, stimulating discussions and valuable suggestions. I also want to thank the ZFITTER team for their longterm collaboration; its start in 1985 was my first contact to Standard Model physics and inspired my interest in precision measurements.

\section{References}

[1] Glashow S L 1961 Nucl. Phys. 22579 Weinberg S 1967 Phys. Rev. Lett. 191264

Salam A 1968 Weak and electromagnetic interactions Originally printed in Svartholm: Elementary Particle Theory, Proc. Nobel Symp. (Lerum, Sweden, Stockholm 1968) pp 367-77

[2] Veltman M J G 1968 Nucl. Phys. B 7637 't Hooft G 1971 Nucl. Phys. B 35167

't Hooft G and Veltman M J G 1972 Nucl. Phys. B 44189 't Hooft G and Veltman M J G 1972 Nucl. Phys. B 50318

[3] Hasert F J et al 1973 Phys. Lett. B 46121

[4] Hasert F J et al 1973 Phys. Lett. B 46138

[5] Arnison G et al 1983 Phys. Lett. B 122103 Banner M et al 1983 Phys. Lett. B 122476

[6] Arnison G et al 1983 Phys. Lett. B 126398 Bagnaia P et al 1983 Phys. Lett. B 129130

[7] Barate R et al 2003 Phys. Lett. B 56561

[8] Gross D J and Wilczek F 1973 Phys. Rev. Lett. 301343 Politzer H D 1973 Phys. Rev. Lett. 301346

[9] Amsler C et al 2008 Phys. Lett. B 6671

[10] Bardin D Y, Khristova P K and Fedorenko O M 1980 Nucl. Phys. B 175435

Bardin D Y, Khristova P K and Fedorenko O M 1982 Nucl. Phys. B 1971

[11] Burgers G and Jegerlehner F $1989 \Delta r$ or the relation between the electroweak couplings and the weak vector boson masses, in Z Physics At LEP 1. Proc. Workshop (Geneva, Switzerland, 4-5 September 1989) vol 1: Standard Physics, CERN 89-08 ed G Altarelli et al (Geneva, Switzerland: CERN) p 55

[12] Ross D A and Veltman M J G 1975 Nucl. Phys. B 95135

[13] Veltman M J G 1977 Nucl. Phys. B 12389

[14] Akhundov A A, Bardin D Y and Riemann T 1986 Nucl. Phys. B 2761

[15] Beenakker T and Hollik W 1988 Z. Phys. C 40141

[16] Bernabeu J, Pich A and Santamaria A 1988 Phys. Lett. B 200569

[17] Bernabeu J, Pich A and Santamaria A 1991 Nucl. Phys. B 363326

[18] Fleischer J, Tarasov O V and Jegerlehner F 1993 Phys. Lett. B 319249

[19] Fleischer J et al 1992 Phys. Lett. B 293437

[20] Chetyrkin K G, Kwiatkowski A and Steinhauser M 1993 Mod. Phys. Lett. A 82785

[21] Buchalla G and Buras A J 1993 Nucl. Phys. B 398285

[22] Degrassi G 1993 Nucl. Phys. B 407271

[23] Bardin D Y et al 1989 Z. Phys. C 44493

Bardin D Y et al 1990 Comput. Phys. Commun. 59303 
Bardin D Y et al 1991 Nucl. Phys. B 351

Bardin D Y et al 1991 Phys. Lett. B 255290

Bardin D Y et al 1992 (arXiv:hep-ph/9412201)

Bardin D Y et al 2001 Comput. Phys. Commun. 133229

Kobel M et al 2000 Two-fermion production in electron-positron collisions (arXiv:hep-ph/0007180)

Arbuzov A B et al 2006 Comput. Phys. Commun. 174728

[24] Montagna G et al 1993 Nucl. Phys. B 4013

Montagna G et al 1993 Comput. Phys. Commun. 76328

Montagna G et al 1996 Comput. Phys. Commun. 93120

Montagna G et al 1999 Comput. Phys. Commun. 117278

[25] Bardin D Y and Passarino G 1999 The Standard Model in the Making: Precision Study of the Electroweak Interactions (Oxford: Clarendon)

[26] Bardin D Y, Leike A, Riemann T and Sachwitz M 1988 Phys. Lett. B 206539

[27] Degrassi G and Gambino P 2000 Nucl. Phys. B 5673

[28] Djouadi A and Gambino P 1995 Phys. Rev. D 51218

[29] Czarnecki A and Kuhn J H 1996 Phys. Rev. Lett. 773955

[30] LEP Design Report 1984 Internal Report, CERN-LEP-84-01 The main features of LEP have been reviewed by S Myers and E Picasso 1990 Contemp. Phys. 31387

Brandt D et al 2000 Rep. Prog. Phys. 63939

A useful retrospective view of the accelerator is presented in R Assmann, M Lamont and S Myers 2002 Nucl. Phys. Proc. Suppl. 109B 17

[31] SLAC Linear Collider Conceptual Design Report 1980 Internal Report SLAC-R-229

[32] The ALEPH, DELPHI, L3, OPAL, SLD Collaborations, the LEP Electroweak Working Group, the SLD Electroweak and Heavy Flavour Groups 2006 Phys. Rep. 427257

[33] Jadach S 2003 Theoretical error of luminosity cross section at LEP (arXiv:hep-ph/0306083)

[34] Decamp D et al 1990 Z. Phys. C 48365 Decamp D et al 1992 Z. Phys. C 531 Buskulic D et al 1993 Z. Phys. C 6071 Buskulic D et al 1994 Z. Phys. C 62539 Barate R et al 2000 Eur. Phys. J. C 141

[35] Abreu P et al 1991 Nucl. Phys. B 367511 Abreu P et al 1994 Nucl. Phys. B 4173 Abreu P et al 1994 Nucl. Phys. B 418403 Abreu P et al 2000 Eur. Phys. J. C 16371

[36] Adeva B et al 1991 Z. Phys. C 51179 Adriani O et al 1993 Phys. Rep. 2361 Acciarri M et al 1994 Z. Phys. C 62551 Acciarri M et al 2000 Eur. Phys. J. C 161

[37] Alexander G et al 1991 Z. Phys. C 52175 Acton P D et al 1993 Z. Phys. C 58219 Akers R et al 1994 Z. Phys. C 6119 Abbiendi G et al 2001 Eur. Phys. J. C 19587

[38] Bederede D et al 1995 Nucl. Instrum. Methods A 365117 Brock I C et al 1996 Nucl. Instrum. Methods A 381236 Abbiendi G et al 2000 Eur. Phys. J. C 14373

[39] Ternov I M and Sokolov A A 1964 Sov. Phys._Dokl. 81203

[40] Assmann R et al 1999 Eur. Phys. J. C 6187 Arnaudon L et al 1995 Z. Phys. C 6645 Arnaudon L et al 1992 Phys. Lett. B 284431 Knudsen L et al 1991 Phys. Lett. B 27097

[41] Assmann R et al 2000 Spin dynamics in LEP with $40 \mathrm{GeV}-100 \mathrm{GeV}$ beams AIP Conf. Proc. 570169

[42] Field R C et al 1998 IEEE Trans. Nucl. Sci. 45670

[43] Swartz M L 1998 Phys. Rev. D 58014010

[44] Band H R, Rowson P C and Wright T R 2000

[45] Brau J et al (ed) 2007 ILC Reference Design Report vol 1-Executive Summary (arXiv:0712.1950)

[46] Assmann R W et al $2000 \mathrm{~A} 3-\mathrm{TeV} \mathrm{e}^{+} \mathrm{e}^{-}$linear collider based on CLIC technology CERN-2000-008

[47] Accomando E et al 2004 Physics at the CLIC multi-TeV linear collider, arXiv:hep-ph/0412251
[48] Moortgat-Pick G A et al 2008 Phys. Rep. 460131

[49] Mane S R, Shatunov Y M and Yokoya K 2005 Rep. Prog. Phys. 681997

[50] Heister A et al 2001 Eur. Phys. J. C 20401 Buskulic D et al 1996 Z. Phys. C 69183 Buskulic D et al 1993 Z. Phys. C 59369 Decamp D et al 1991 Phys. Lett. B 265430

[51] Abreu P et al 2000 Eur. Phys. J. C 14585 Abreu P et al 1995 Z. Phys. C 67183

[52] Acciarri M et al 1998 Phys. Lett. B 429387 Acciarri M et al 1994 Phys. Lett. B 341245 Adriani O et al 1992 Phys. Lett. B 294466

[53] Abbiendi G et al 2001 Eur. Phys. J. C 211 Alexander G et al 1996 Z. Phys. C 72365 Akers R et al 1995 Z. Phys. C 651 Alexander G et al 1991 Phys. Lett. B 266201

[54] Abe K et al 1993 Phys. Rev. Lett. 702515 Abe K et al 1994 Phys. Rev. Lett. 7325 Abe K et al 1997 Phys. Rev. Lett. 782075 Abe K et al 2000 Phys. Rev. Lett. 845945

[55] Abe Ket al 2001 Phys. Rev. Lett. 861162

[56] Abe K et al 1997 Phys. Rev. Lett. 79804

[57] Abreu P et al 2000 Eur. Phys. J. C 14613 Abreu P et al 1995 Z. Phys. C 67

[58] Abe K et al 2000 Phys. Rev. Lett. 855059

[59] Ackerstaff K et al 1997 Z. Phys. C 76387

[60] Mönig K 1998 Rep. Prog. Phys. 61999

[61] Abbaneo D et al 1998 Eur. Phys. J. C 4185

[62] Leike A, Riemann T and Rose J 1991 Phys. Lett. B 273513

[63] Riemann T 1992 Phys. Lett. B 293451

[64] Kirsch S and Riemann T 1995 Comput. Phys. Commun. 8889

[65] Miyabayashi K et al 1995 Phys. Lett. B 347171

[66] Yusa K et al 1999 Phys. Lett. B 447167

[67] Schael S et al 2007 Eur. Phys. J. C 49411

Abdallah J et al 2006 Eur. Phys. J. C 45589

Acciari M et al 2000 Phys. Lett. B 479101

Achard P et al 2006 Eur. Phys. J. C 471

Ackerstaff K et al 1998 Eur. Phys. J. C 2441 (arXiv:hep-ex/9708024)

Abbiendi G et al 1999 Eur. Phys. J. C 61 (arXiv:hep-ex/9808023)

Abbiendi G et al 2000 Eur. Phys. J. C 13553 (arXiv:hep-ex/9808031)

Abbiendi G et al 2004 Eur. Phys. J. C 33173

[68] Steinhauser M 1998 Phys. Lett. B 429158

[69] Eidelman S and Jegerlehner F 1995 Z. Phys. C 67585

[70] Burkhardt H and Pietrzyk B 1995 Phys. Lett. B 356398

[71] Burkhardt H and Pietrzyk B 2005 Phys. Rev. D 72057501

[72] Swartz M L 1996 Phys. Rev. D 535268

Martin A D and Zeppenfeld D 1995 Phys. Lett. B 345558

Alemany R, Davier M and Höcker A 1998 Eur. Phys. J. C 2123 (arXiv:hep-ph/9703220)

Davier M and Höcker A 1998 Phys. Lett. B 419419

Kuhn J H and Steinhauser M 1998 Phys. Lett. B 437425

Jegerlehner F 1999 Hadronic effects in $(g-2)_{\mu}$ and $\alpha_{\mathrm{QED}}\left(m_{\mathrm{Z}}\right)$ : Status and perspectives (arXiv:hep-ph/9901386)

Erler J 1999 Phys. Rev. D 59054008

Ryskin M G, Martin A D and Outhwaite J 2000 Phys. Lett. B 49269

de Troconiz J F and Yndurain F J 2002 Phys. Rev. D 65093002

Hagiwara K et al 2004 Phys. Rev. D 69093003

[73] de Troconiz J F and Yndurain F J 2005 Phys. Rev. D 71073008

[74] Roos M and James F 1975 Comput. Phys. Commun. 10343

[75] Bethke S 2004 Nucl. Phys. Proc. Suppl. 135345

[76] Kluth S 2006 Rep. Prog. Phys. 691771

[77] Denner A et al 2000 Phys. Lett. B 475127 
[78] Denner A et al 2000 Nucl. Phys. B 58767

[79] Jadach S et al 2000 Phys. Rev. D 61113010

[80] Jadach S et al 2001 Comput. Phys. Commun. 140432

[81] Jadach S et al 2001 Comput. Phys. Commun. 140475

[82] Barate R et al 1997 Phys. Lett. B 401347

[83] Abreu P et al 1997 Phys. Lett. B 397158

[84] Acciarri M et al 1997 Phys. Lett. B 398223

[85] Ackerstaff K et al 1996 Phys. Lett. B 389416

[86] The ALEPH, DELPHI, L3, OPAL, SLD Collaborations, the LEP Electroweak Working Group, the SLD Electroweak and Heavy Flavour Groups 2006 A combination of preliminary electroweak measurements and constraints on the Standard Model (arXiv:hep-ex/0612034)

[87] Abreu P et al 1997 Phys. Lett. B 397158

[88] Acciari M et al 1997 Phys. Lett. B 398223

[89] Ackerstaff K et al 1996 Phys. Lett. B 389416

[90] The LEP Electroweak Working Group, the Tevatron Electroweak Working Group and the SLD electroweak and heavy flavour groups, Precision Electroweak Measurements and Constraints on the Standard Model 2008, CERN-PH-EP/2008-020 (arXiv:0811.4682 [hep-ex])

[91] Abe F et al 1990 Phys. Rev. Lett. 652243 Abe F et al 1991 Phys. Rev. D 432070 Abe F et al 1995 Phys. Rev. Lett. 7511 Abe F et al 1995 Phys. Rev. D 524784 Affolder A A et al 2001 Phys. Rev. D 64052001

[92] Abbott B et al 1998 Phys. Rev. Lett. 803008 Abbott B et al 2000 Phys. Rev. Lett. 84222 Abazov V M et al 2002 Phys. Rev. D 66012001 Abbott B et al 2000 Phys. Rev. D 62092006

[93] Affolder A A et al 2000 Phys. Rev. Lett. 853347

[94] Abazov V M et al 2002 Phys. Rev. D 66032008

[95] Abazov V M et al 2004 Phys. Rev. D 70092008

[96] The Tevatron Electroweak Working Group 2009 Updated combination of CDF and $\mathrm{D} \varnothing$ results for the mass of the $\mathrm{W}$ boson (arXiv:0908.1374 [hep-ex])

[97] The LEP Electroweak Working Group, the Tevatron Electroweak Working Group and the SLD electroweak and heavy flavour groups 2009 Precision Electroweak Measurements and Constraints on the Standard Model CERN-PH-EP/2009-023 (arXiv:0911.2604 [hep-ex])

[98] The LEP Electroweak Working Group, the Tevatron Electroweak Working Group and the SLD electroweak and heavy flavour groups 2010 Precision Electroweak Measurements and Constraints on the Standard Model Update Summer 2010 http://lepewwg.web.cern.ch/ LEPEWWG/

[99] The Tevatron Electroweak Working Group 2010 Combination of CDF and DØ Results on the Width of the W boson (arXiv:1003.2826 [hep-ex])

[100] Abe F et al 1995 Phys. Rev. Lett. 742626

[101] Abachi S et al 1995 Phys. Rev. Lett. 742632

[102] Wagner W 2005 Rep. Prog. Phys. 682409

[103] Tevatron Electroweak Working Group 2010 Combination of CDF and DØ Results on the Mass of the Top Quark (arXiv:1007.3178 [hep-ex])

[104] Wood C S et al 1997 Science 2751759

[105] Wieman C E and Bennett S C 1999 Phys. Rev. Lett. 822484

[106] Derevianko A and Porsev S G 2007 Eur. Phys. J. A 32517

[107] Rosner J L 2002 Phys. Rev. D 65073026

[108] Anthony P L et al 2004 Phys. Rev. Lett. 92181602

[109] Anthony P L et al 2005 Phys. Rev. Lett. 95081601

[110] Czarnecki A and Marciano W J 2000 Int. J. Mod. Phys. A 152365

[111] Paschos E A and Wolfenstein L 1973 Phys. Rev. D 791

[112] Zeller G P et al 2003 Phys. Rev. Lett. 88091802 Zeller G P et al 200390239902 (erratum)

[113] van Ritbergen T and Stuart R G 1999 Phys. Rev. Lett. 82488 van Ritbergen T and Stuart R G 2000 Nucl. Phys. B 564343

Steinhauser M and Seidensticker T 1999 Phys. Lett. B 467271

[114] Zeller G P et al 2002 Phys. Rev. D 65111103

[115] McFarland K S and Moch S 2003 Conventional physics explanations for the $\mathrm{NuTeV} \sin ^{2} \theta_{\mathrm{W}}$ (arXiv:hep-ph/0306052)

[116] Olness F et al 2005 Eur. Phys. J. C 40145

[117] Kretzer S et al 2004 Phys. Rev. Lett. 93041802

[118] Martin A D, Roberts R G, Stirling W J and Thorne R S 2005 Eur. Phys. J. C 39155

[119] Flambaum V V and Ginges J S M 2004 Phys. Rep. 39763

[120] The TEVNPH Working Group of the CDF and DØCollaborations 2010 Combined CDF and DØ Upper Limits on Standard Model Higgs-Boson Production with up to $6.7 \mathrm{fb}^{-1}$ of Data (arXiv:1007.4587 [hep-ex])

Aaltonen $\mathrm{T}$ et al and the CDF and DØ collaboration 2010 Phys. Rev. Lett. 104061802

[121] Aarons G et al 2007 International Linear Collider Reference Design Report volume 2: Physics at the ILC (arXiv:0709.1893)

[122] Jegerlehner F 2006 Nucl. Phys. Proc. Suppl. 16222

[123] Erler J et al 2000 Phys. Lett. B 486125

[124] Peskin M E and Takeuchi T 1990 Phys. Rev. Lett. 65964 Peskin M E and Takeuchi T 1992 Phys. Rev. D 46381

[125] Caso C et al (Particle Data Group) 1998 Eur. Phys. J. C 31

[126] Altarelli G and Barbieri R 1991 Phys. Lett. B 253161 Altarelli G, Barbieri R and Jadach S 1992 Nucl. Phys. B 3693

Altarelli G, Barbieri R and Caravaglios F 1993 Nucl. Phys. B 4053

Altarelli G, Barbieri R and Caravaglios F 1993 Phys. Lett. B 314357

Altarelli G, Barbieri R and Caravaglios F 1995 Phys. Lett. B 349145

Altarelli G, Barbieri R and Caravaglios F 1998 Int. J. Mod. Phys. A 131031

[127] Barate R et al 2000 Eur. Phys. J. C 12183

[128] Abreu P et al 1999 Eur. Phys. J. C 11383

[129] Abdallah J et al 2009 Eur. Phys. J. C 601

[130] Acciarri J et al 2000 Phys. Lett. B 48571

[131] Abbiendi G et al 2000 Eur. Phys. J. C 1641

[132] Abbiendi G et al 2005 Phys. Lett. B 609212

[133] LEPEWWG Heavy Flavour at LEP-II Subgroup 2000 Combination of heavy flavour measurements at LEP-II, LEP2FF/00-02

[134] Eichten E, Lane K D and Peskin M E 1983 Phys. Rev. Lett. $\mathbf{5 0} 811$

[135] Kroha H 1992 Phys. Rev. D 4658

[136] Adloff C et al 2003 Phys. Lett. B 56835

[137] Abe F et al 1997 Phys. Rev. Lett. 792198

[138] Abbott B et al 1999 Phys. Rev. Lett. 822457

[139] Kilian W and Zerwas P M 2005 ECONF, C0508141:PLEN0003 (arXiv:hep-ph/0601217)

[140] Wess J 2009 From Symmetry to Supersymmetry (arXiv:0902.2201 [hep-th])

[141] Buchmüller O et al 2010 Phys. Rev. D 81035009 Phys.Rev.D81:035009

[142] Heinemeyer S, Hollik W, Weber A M and Weiglein G 2008 JHEP04(2008)039 (arXiv:0710.2972)

[143] Pape L and Treille D 2006 Rep. Prog. Phys. 692843

[144] Arkani-Hamed N, Dimopoulos S and Dvali G R 1998 Phys. Lett. B 429263

[145] Antoniadis I et al 1998 Phys. Lett. B 436257

[146] Zhang R, Han T and Lykken J D 1999 Phys. Rev. D 59105006

[147] Shrock R and Nussinov S 1999 Phys. Rev. D 59105002

[148] Heister A et al 2003 Eur. Phys. J. C 281

Abdallah J et al 2005 Eur. Phys. J. C 38395 
Achard P et al 2004 Phys. Lett. B 58716

[149] Aaltonen T et al 2008 Phys. Rev. Lett. 101181602 Abulencia A et al 2006 Phys. Rev. Lett. 97171802

[150] Giudice G F, Rattazzi R and Wells J D 1999 Nucl. Phys. B 5443

[151] Hewett J L 1999 Phys. Rev. Lett. 824765

[152] Rizzo T G 1999 Phys. Rev. D 59115010

[153] Abazov V M et al 2009 Phys. Rev. Lett. 102051601 Abazov V M et al 2009 Phys. Rev. Lett. 103191803

[154] Randall L and Sundrum R 1999 Phys. Rev. Lett. 834690

[155] Aaltonen T et al 2009 Phys. Rev. Lett. 102091805

[156] Appelquist T, Cheng H and Dobrescu B A 2001 Phys. Rev. D 64035002

[157] Weinberg S 1957 Phys. Rev. 1061301 Weinberg S 1979 Phys. Rev. D 191277

[158] For a review of strong electroweak symmetry breaking see C Hill and E Simmons 2003 Phys. Rep. 381235

[159] Arkani-Hamed N et al 2002 JHEP08(2002)021 (arXiv:hep-ph/0206020)

Arkani-Hamed N et al 2002 JHEP07(2002)034 (arXiv:hep-ph/0206021)

[160] Cheng H and Low I 2003 JHEP09(2003)051 (arXiv:hep-ph/0308199)

Cheng H and Low I 2004 JHEP08(2004)061 (arXiv:hep-ph/0405243)

Low I 2004 JHEP10(2004)067 (arXiv:hep-ph/0409025)
[161] Langacker P 2010 AIP Conf. Proc. 120055 (arXiv:0909.3260)

Langacker P 2008 Rev. Mod. Phys. 811199 (arXiv:0801.1345)

Leike A 1999 Phys. Rep. 317143

Hewett J L and Rizzo T G 1989 Phys. Rep. 183193

[162] Abreu P et al 1995 Z. Phys. C 65603

Adriani O et al 1993 Phys. Lett. B 306187

[163] Aaltonen T et al 2009 Phys. Rev. Lett. 102031801

DØ Collaboration 2009 Search for high-mass narrow resonances in the di-electron channel at $\mathrm{D} \emptyset, \mathrm{D} \emptyset$ Note 5923-CONF

[164] ATLAS Collaboration 1999 Physics TDR, 1999, CERN/LHCC 99-14 and CERN/LHCC 99-15

[165] CMS Collaboration 2007 J. Phys. G: Nucl. Part. Phys. 34995 CMS Collaboration 2006 Physics TDR. CERN/LHCC 2006-021

[166] Riemann S 2006 Fermion pair production at a linear collider: a sensitive tool for new physics searches LC-TH-2001-007

[167] Weiglein G et al 2006 Phys. Rep. 42647

[168] Czakon M, Gluza J and Hejczyk J 2003 Nucl. Phys. Proc. Suppl. 116230

Czakon M, Gluza J and Hejczyk J 2002 Nucl. Phys. B 642157

Czakon M, Gluza J, Jegerlehner F and Zralek M 2000 Eur Phys. J. C 13275 (arXiv:hep-ph/9909242) 